\title{
The dynamic gastric environment and its impact on drug and formulation behaviour
}

\author{
Jens Van Den Abeele ${ }^{a, *}$, Jari Rubbens ${ }^{b, *}$, Joachim Brouwers ${ }^{c}$, Patrick Augustijns ${ }^{d}$
}

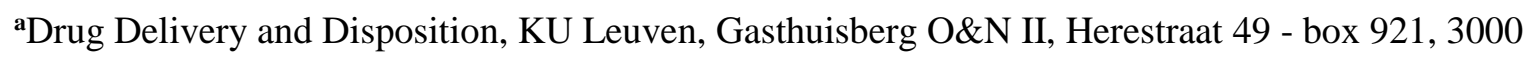

Leuven, Belgium - jens.vandenabeele@kuleuven.be

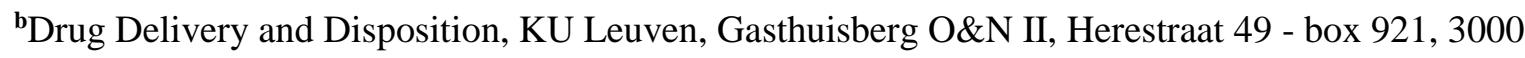

Leuven, Belgium - jari.rubbens@ @uleuven.be

${ }^{\mathrm{c}}$ Drug Delivery and Disposition, KU Leuven, Gasthuisberg O\&N II, Herestraat 49 - box 921, 3000

Leuven, Belgium - joachim.brouwers@ kuleuven.be

${ }^{\mathrm{d} D r u g}$ Delivery and Disposition, KU Leuven, Gasthuisberg O\&N II, Herestraat 49 - box 921, 3000

Leuven, Belgium - patrick.augustijns@kuleuven.be

${ }^{*}$ These authors should both be considered as first authors

Corresponding author:

Patrick Augustijns

Drug Delivery and Disposition, KU Leuven, Gasthuisberg O\&N II, Herestraat 49 - box 921, 3000

Leuven, Belgium

Tel: +3216330301 Fax: +3216330305

patrick.augustijns@kuleuven.be 


\begin{abstract}
Before being absorbed in the small intestine and/or colon, orally administered drugs inevitably need to pass through the stomach. Hence, it seems reasonable that the residence of a dosage form in the gastric environment, however brief it may be, may influence drug disposition further down the gastrointestinal tract and may potentially impact systemic exposure to a drug of interest. However, research efforts in the past mainly focused on drug disposition at the level of the intestine, i.e. the main site of absorption, hereby disregarding or oversimplifying the stomach's contribution to gastrointestinal drug disposition. In the first part of this review, the complexity of the stomach with regard to anatomy, physiology and gastric fluid composition is emphasized. Between-population differences in gastric functioning and physicochemical characteristics of gastric fluids are discussed. The second part of this review focuses on several of the processes to which a dosage form can be exposed during its passage through the stomach and the implications for gastrointestinal drug behaviour and systemic drug disposition. Finally, the influence of real-life dosing conditions on drug disposition is discussed in the context of the stomach.
\end{abstract}

\title{
Key words ( 3 à 6 woorden)
}

Stomach, gastrointestinal tract, drug disposition, biopharmaceutics, oral drug delivery 


\section{Introduction}

In order to reduce drug attrition during the development of orally delivered dosage forms, an in-depth understanding of the gastrointestinal behaviour of said dosage forms is warranted. In doing so, drug development may shift from a trial and error- to an evidence-based approach, saving valuable time and expenses (Lennernas et al., 2014). Before being absorbed in the small intestine and/or colon, orally administered drugs inevitably need to pass through the stomach. Hence, it seems reasonable that the residence of a dosage form in the gastric environment, however brief it may be, may influence drug disposition further down the gastrointestinal (GI) tract and may potentially impact systemic exposure to a drug of interest. However, research efforts in the past have focused on drug disposition at the level of the intestine, i.e. the main site of absorption, hereby disregarding or oversimplifying the stomach's contribution to GI drug disposition. In recent years, increasing attention has been directed to the stomach, as illustrated by efforts to better mimic the gastric environment in order to improve the significance of in vitro tools used in oral drug development (Chessa et al., 2014; Minekus, 2015). In order to further optimize the biorelevance of such tools, a better understanding of all gastric variables and processes potentially influencing a dosage form's performance in the GI tract is indispensable. Therefore, this review compiles literature reports relevant to the topic of gastric drug behaviour, focusing on (i) the complexity and variability of gastric anatomy and physiology and (ii) the stomach's contribution to the disposition of orally administered dosage forms.

\section{Anatomy}

The stomach is a hollow, distensible organ located in the left upper quadrant of the peritoneal cavity (Mahadevan, 2014). As a result of the distinct curving of the gastric wall, which is more pronounced on the left compared to the right border (i.e. 'greater and lesser curvature', respectively), the stomach is classically represented as a J-shaped organ (Fig. 1). However, due to its distensibility, stomach shape can vary considerably depending on numerous factors, e.g. degree of fullness, body position and body type (Burdan et al., 2012; Ellis, 2011). Orally ingested content reaches the proximal stomach via the esophagus, crossing the gastro-esophageal junction (i.e. 'cardia'). The distal end of the stomach is connected to the duodenum, enabling further transit of content along the GI tract. This connection is 
characterized by the presence of a muscular ring, i.e. the 'pyloric sphincter' or 'pylorus' (Skandalakis et al., 2009; Soybel, 2005). The region spanned by cardia and pylorus can further be divided into three anatomically distinct regions: (i) the 'fundus', situated to the upper left of the cardia; (ii) the 'corpus', comprising the region distally from the cardia up to the 'incisura angularis', a distinct landmark on the lesser curvature; (iii) the 'antrum', ranging from the incisura angularis to the pylorus. Apart from the incisura, corpus and antrum can easily be differentiated by the presence of mucosal folds (i.e. 'rugae') which are less distinct (flatter) in the antrum (Soybel, 2005).

\subsection{Deviating gastric anatomy and implications for drug disposition}

Burdan et al. reported a vast amount of anatomical variations in a large group of patients, ranging from hiatal hernias (most common) over gastric cascades to the (partial) absence of the organ after gastrectomy (Burdan et al., 2012). These deviations from normal gastric anatomy may have an impact on the disposition of orally administered drugs. For instance, in the specific case of the so-called 'cascade stomach' or 'gastric cascade', the greater curvature of the stomach is significantly indented, anatomically dividing the stomach in two main parts, i.e. a corpus-antrum region and a fundic reservoir ('pouch') (Burdan et al., 2012; Ostensen et al., 1983; Schouten et al., 2007). Although the reported incidence of cascade stomach is low (range: 4 - 14\%), the condition may often go undiagnosed resulting in an underestimation of its true incidence (Ostensen et al., 1983; Schouten et al., 2007; Sirakov et al., 1994). Congenital, functional (e.g. excessive swallowing of air) and organic factors (e.g. abnormal adhesion to the diaphragm, ulceration, tumors) have been hypothesized to cause this anatomical variation (Kusano et al., 2012; Ostensen et al., 1983; Schouten et al., 2007). Furthermore, obesity is thought to be a risk factor in the development of cascade stomach. Kusano et al. reported modestly higher Body Mass Index (BMI) values in patients with cascade stomach compared to controls. Increased external pressure on the stomach due to the relatively high intra-abdominal fat content in an obese population may cause pouch formation in these subjects (Burdan et al., 2012; Kusano et al., 2012). Several authors have suggested a possible link between cascade stomach and the presence of upper GI symptoms. For instance, gastric acid may pool in the fundus hereby increasing the risk of acid reflux in the esophagus (Burdan et al., 2012; Kusano et al., 2012; Schouten et al., 2007). Kusano et al. further 
hypothesized that gastric emptying (GE) may be accelerated in patients with cascade stomach due to fluid/food build-up in the fundus. In this scenario, the pooled material rapidly cascades into the corpus when the fundus is completely filled, hereby accelerating gastric emptying ('cup and spill') (Kusano et al., 2012). In the case of oral drug administration, inhomogeneous drug distribution and/or altered gastric emptying may contribute to inter-patient variability in systemic drug exposure. However, the influence of cascade stomach and other anatomical variations of the human stomach on drug disposition has not been the subject of further study and thus remains to be elucidated.

Secondly, gastric resection as part of a bariatric procedure constitutes a significant change in GI anatomy. According to figures from the World Health Organization (WHO), the worldwide incidence of obesity has increased twofold compared to 1980, with more than 600 million people (13\% of the global population) categorized as being obese $\left(\mathrm{BMI} \geq 30 \mathrm{~kg} / \mathrm{m}^{2}\right)$ in 2014 (WHO, 2015). In parallel, the number of bariatric surgeries performed as a means of inducing weight loss has skyrocketed (Angrisani et al., 2015). An in-depth description of the different bariatric procedures is outside the scope of this review and can be found elsewhere (Lannoo and Dillemans, 2014; Neff et al., 2013; Piche et al., 2015; Titus et al., 2013). Depending on the extent and site of resection (subtotal vs. total gastrectomy, proximal vs. distal resection), the gastric environment can be markedly affected, including changes in gastric volume, acidity and motility. As a result, processes such as formulation disintegration, drug dissolution (in the case of $\mathrm{pH}$-dependency) and gastric emptying may be altered, resulting in unfavorable, i.e. subtherapeutic or toxic, drug pharmacokinetics. For example, mean gastric emptying half-time $\left(\mathrm{GE} \mathrm{t}_{1 / 2}\right)$ was markedly accelerated in bariatric surgery patients (sleeve gastrectomy with partial antral resection) compared to healthy controls, both for liquids $(13.6 \pm 11.9$ vs. $34.9 \pm 24.6 \mathrm{~min})$ and solids $(38.3 \pm 18.8$ vs. $78 \pm 15.0 \mathrm{~min}$ ) (Braghetto et al., 2009). Such changes may particularly be of interest in the case of drugs with a narrow therapeutic window (De Smet et al., 2013; Greenblatt and Greenblatt, 2015; Padwal et al., 2010; Quercia et al., 2014; Titus et al., 2013). Hamad et al. reported decreased bioavailability of serotonin reuptake inhibitors (SRIs) one month after Roux-and-Y gastric bypass (RYGB) compared to pre-RYGB. For some of these SRIs, this difference may be the result of decreased solubility in gastric fluids due to a decrease in gastric acidity post-RYGB (Hamad et al., 2012). However, as these 
procedures often include bypassing part of the small intestine, other factors (e.g. decrease in GI transit time, intestinal metabolism and available intestinal absorptive area) may contribute to observed changes in oral drug bioavailability after bariatric surgery (Padwal et al., 2011; Skottheim et al., 2009).

\section{Gastric physiology}

\subsection{Blood supply, lymphatic flow and innervation}

Branches of the celiac artery ensure adequate blood supply and drainage of blood to the stomach and venous blood drains into the portal system (Ellis, 2011; Mahadevan, 2014; Trowers and Tischler, 2014a). Similarly, lymphatic fluid is drained from lymph nodes distributed across the stomach and collected in the celiac lymph nodes (Farre and Tack, 2013; Mahadevan, 2014; Soybel, 2005; Trowers and Tischler, 2014a). The stomach is characterized by extensive intrinsic innervation. As part of the GI tract, the stomach receives stimuli from the 'Enteric Nervous System (ENS)' which acts largely independent from the 'Central Nervous System $(C N S)$ '. Due to the vast amount of neurons (approx. 200 - 600 million, an amount similar to the spinal cord), the ENS is sometimes referred to as the 'little brain'. ENS neurons are organized in ganglia, which in turn are grouped in two networks: one in the submucosa (Meissner's or submucosal plexus) and one between the smooth muscle layers (Auerbach's or myenteric plexus). Intrinsic innervation is involved in the regulation of several GI functions such as smooth muscle contractility, gastric emptying, blood flow and regulation of GI secretory activity (Ellis, 2011; Furness, 2006; Sasselli et al., 2012; Schubert and Peura, 2008; Trowers and Tischler, 2014b). Apart from intrinsic innervation, gastric functions are regulated by autonomous extrinsic innervation, sympathetic as well as parasympathetic (Furness, 2006; Mahadevan, 2014; Soybel, 2005; Trowers and Tischler, 2014a). Within this autonomic 'gut-brain axis', it is mainly the input of the vagal nerve which is involved in the regulation of gastric motility and secretion (Lakkireddy et al., 2015; Mahadevan, 2014; Murakami et al., 2013).

\subsection{Gastric secretions}

The gastric secretory function is executed by gastric glands along the gastric wall (Fig. 2). In fasted subjects, Dubois et al. reported mean secretion rates of approximately one mL.min ${ }^{-1}$ (Dubois et al., 
1977). In an effort to re-establish fasted state conditions and digest food components, secretion of gastric fluid components (e.g. water, acid, enzymes, mucus) greatly increases in response to a meal (CastroCombs et al., 2014; Dubois et al., 1977; Feldman et al., 1996; Malagelada et al., 1976; Szafran et al., 1978). Similar to the distinction between several anatomical regions in the stomach, one could also make a distinction based on secretory function, i.e. an acid-secreting (fundus-corpus) and a non-acid-secreting region (antrum), often referred to as the oxyntic gland area and the pyloric gland area, respectively (Chu and Schubert, 2013; Schubert and Peura, 2008; Soybel, 2005).

Parietal cells are the main cell-type present in the oxyntic gland mucosa (Fig. 2). These cells are responsible for the secretion of hydrochloric acid ( 'gastric acid') via $\mathrm{H}^{+} / \mathrm{K}^{+}$ATPase-mediated exchange of intracellular $\mathrm{H}^{+}$with intraluminal $\mathrm{K}^{+}$ions. Gastric acid secretion is mediated by the complex interplay between both endocrine and neural (vagal) stimuli. Histamine, secreted by enterochromaffin-like (ECL) cells of the oxyntic gland mucosa, and gastrin, secreted by G-cells situated in the antral region, represent the main gut hormones that stimulate gastric acid secretion both directly and indirectly. On the other hand, acid secretion is inhibited by secretion of somatostatin, produced in D-cells present in all gastric glands (Fig. 2) (Chu and Schubert, 2013; Schubert and Peura, 2008; Soybel, 2005). In fasted state, gastric acid secretion rates ranging between $0.03-0.07 \mathrm{mEq} \mathrm{H}^{+}$. $\mathrm{min}^{-1}$ have been reported (Charman et al., 1997; Dubois et al., 1977). In response to meal intake, secretion rates rise significantly in an effort to re-acidify gastric contents (Dubois et al., 1977; Malagelada et al., 1976; Szafran et al., 1978). Predominant secretion of gastric acid at the level of corpus and fundus explains the inhomogeneous distribution of gastric acid after a meal. Indeed, several authors have reported the presence of a layer of acid ('acid pocket') on top of the ingested meal near the gastro-esophageal junction, which is linked to gastro-esophageal reflux (Goetze et al., 2009; Simonian et al., 2005; Steingoetter et al., 2015).

Mucus neck cells and surface mucus cells, present in all secretory glands, secrete mucins in the gastric lumen (Fig. 2). Mucins are glycoproteins which form a continuous gel network along the gastric wall, protecting gastric mucosa from (i) autodigestion by endogenous gastric acid and pepsin and (ii) exogenous aggression (e.g. bacteria). By establishing a $\mathrm{pH}$ gradient across the mucus layer via bicarbonate secretion, back-diffusing protons are neutralized and pepsin is inactivated before reaching 
the gastric wall (Allen and Flemstrom, 2005; Cone, 2009; Ensign et al., 2012). Castro-Combs et al. determined mucin secretion rates by aspirating gastric contents from healthy subjects for one hour. Mean basal mucin secretion rate amounted to $24.6 \pm 4.9 \mathrm{mg} \cdot \mathrm{h}^{-1}$ and subsequently increased after simulation of fed state conditions via pentagastrin administration (32.1 $\left.\pm 3.3 \mathrm{mg} . \mathrm{h}^{-1}\right)$ (Castro-Combs et al., 2014).

Furthermore, the stomach secretes several enzymes, such as pepsin and gastric lipase. These enzymes are crucial for the digestion of respectively proteins and triglycerides present in a meal, breaking down meal components into essential nutrients which can then be absorbed in the small intestine (Fig. 2). Pepsin is secreted as its inactive precursor pepsinogen mainly by gastric chief cells present in oxyntic glands. In the presence of gastric acid, the precursor is subsequently converted to its active, proteolytic form (pepsin) (al-Janabi et al., 1972; Gritti et al., 2000; O'Connor and O'Morain, 2014). Similar to the secretion of gastric acid, pepsinogen secretion is controlled by numerous stimuli. Nervous (acetylcholine), endocrine (somatostatin) and autoregulatory impulses ensure adequate stimulation/inhibition of pepsinogen secretion in response to dynamic changes in the gastric lumen (Gritti et al., 2000; Ito et al., 2008). For instance, meal intake stimulates pepsin output. Feldman et al. observed a 2.5-fold increase in mean pepsin output rate after pentagastrin administration compared to basal output rate $\left(4.8 \pm 2.3\right.$ vs. $\left.2.0 \pm 1.3 \mathrm{IU} .10^{3} .15 \mathrm{~min}^{-1}\right)$ (Feldman et al., 1996). Using immunocytolocalization, Moreau et al. traced back the secretion of gastric lipase to the chief cells of the fundic mucosa, similar to the secretion of pepsinogen (Moreau et al., 1989). Logically, gastric lipase secretion is stimulated by meal intake in order to be able to cope with the increased presence of triglycerides in the stomach. Similar as for the secretion of $\mathrm{H}^{+}$and pepsinogen, Szafran et al. observed increasing rates of gastric lipase secretion with increasing dose of pentagastrin administered (Szafran et al., 1978).

\subsection{Gastric fluid characteristics}

\subsubsection{Gastric volume}

Gastric secretions, ingested fluids/solids, reflux of duodenal contents and emptying of gastric contents from the stomach in the duodenum all determine the volume of gastric contents at a given time. Gastric fluid composition is diverse, consisting of both liquid (e.g. water, gastric acid, enzymes, electrolytes, 
ingested fluids) as well as viscous (e.g. mucus, swallowed saliva) and solid material (e.g. food remnants).

Magnetic Resonance Imaging (MRI) is often applied in order to estimate gastric volumes in man (Goetze et al., 2006; Hoad et al., 2015). By forming a magnetic field around the patient, mobile hydrogen groups, present in e.g. water, are excited and emit a radio signal upon return to their equilibrium state. Capturing this signal allows for the estimation of gastric water volume (Stark et al., 2007). Using this technique, several authors quantified gastric volume in fasted, healthy volunteers, reporting mean residual volumes ranging between 31 - $45 \mathrm{~mL}$, slightly higher than an earlier assessment by Dubois et al. in $1977(24 \pm 5$ mL; Table 1) (Dubois et al., 1977; Goetze et al., 2009; Koziolek et al., 2014b; Mudie et al., 2014; Schiller et al., 2005).

In order to maintain basal intragastric pressure, the stomach is capable of greatly expanding its total volume in response to a meal, often referred to as 'gastric accommodation' (Fruehauf et al., 2011; Goetze et al., 2009; Koziolek et al., 2014b; Schiller et al., 2005). The extent of this accommodation reflex depends on the ingested meal volume, the extent to which gastric secretions are stimulated and the rate at which the meal is emptied from the stomach. Total gastric volumes up to $900 \mathrm{~mL}$ have been reported (Kwiatek et al., 2009). Single-photon emission computed tomography (SPECT) can be used for research purposes in order to investigate total gastric volume and accommodation. While gastric volume can be assessed by using both solid and liquid radioactive markers, gastric accommodation can


visualizing the stomach lining (Bouras, 2002; De Schepper et al., 2004; Simonian et al., 2003; van den Elzen, 2003). Using SPECT imaging, Liau et al. were able to detect changes in pre- and postprandial gastric volume in volunteers treated with either erythromycin (i.v.) or isosorbide (s.l.). Postprandial gastric volumes were markedly lower after erythromycin treatment, while fasting gastric volumes were larger after isosorbide treatment (Liau et al., 2001).

\subsubsection{Acidity of gastric fluids}


Typically, fasted state gastric content is strongly acidic due to the secretion of gastric acid by parietal cells of the oxyntic mucosa. Mean $\mathrm{pH}$ values ranging between 2.5 and 2.9 have been reported in fasted healthy volunteers (Lindahl et al., 1997; Lu et al., 2010; Pedersen et al., 2000; Pedersen et al., 2013). However, median values (range: 1.55 - 1.80) may better reflect 'normal' gastric $\mathrm{pH}$ as mean values may be skewed due to sporadically measured high $\mathrm{pH}$ values in human gastric aspirates (up to $\mathrm{pH}$ 7.5; Table 1) (Dressman et al., 1990; Lindahl et al., 1997; Pedersen et al., 2013; Press et al., 1998). These higher $\mathrm{pH}$ values are most likely the result of (i) duodenogastric reflux of bile and/or (ii) swallowing of saliva (Kalantzi et al., 2006; Lu et al., 2010). Acidity of gastric contents is crucial to prevent bacterial overgrowth of the gastric lumen as well as for the activity of several gastric enzymes (Martinsen et al., 2005; Piper and Fenton, 1965). Thus, if sustained for a long period of time, elevated gastric $\mathrm{pH}$ may result in drastically impaired enzymatic activity and an increased risk of bacterial infections. Postprandially, the meal itself usually determines gastric $\mathrm{pH}$ due to the relatively high volume of the meal compared to the gastric resting volume (Kalantzi et al., 2006; Koziolek et al., 2013; Simonian et al., 2005). For instance, Kalantzi et al. reported a median $\mathrm{pH}$ of 6.4 in gastric fluids aspirated 30 minutes after ingestion of a liquid meal (500 mL Ensure Plus $\left.{ }^{\circledR}\right)$, similar to the $\mathrm{pH}$ of the meal as such (pH 6.6). Stimulated gastric acid secretion and emptying of the meal from the stomach results in re-acidification of gastric content within a few hours. The rate of re-acidification is mainly determined by the buffering capacity of the meal and the rate at which it is emptied from the stomach (Dressman et al., 1990; Kalantzi et al., 2006; Simonian et al., 2005). In the study by Kalantzi et al., median gastric pH did not reach basal levels more than three hours after intake of a high-fat, liquid meal (750 kcal; $\mathrm{pH}_{210 \text { min: }}$.7); whereas Dressman et al. observed gastric $\mathrm{pH}$ values around two within two hours after a solid meal (1000 kcal) (Dressman et al., 1990; Kalantzi et al., 2006).

\subsubsection{Viscosity of gastric fluids}

Mucins are the main constituents determining the viscosity of fasted state gastric fluids. Castro-Combs et al. could link the reduced viscosity of gastric fluids collected from patients with chronic constipation to a significantly lower output of mucins in these patients compared to healthy subjects (Castro-Combs et al., 2014). Mucin monomers are cross-linked via disulfide bonds, forming a polymeric network (i.e. 
mucus layer) along the gastric wall. Part of this mucus layer is in close contact with the gastric mucosa, while a second part is loosely associated with the former part of the mucus layer and responsible for the lubrication of gastric contents (Allen and Flemstrom, 2005; Cone, 2009). Mucus layer thickness (mean thickness: 50 - $200 \mu \mathrm{m}$, Table 1) is susceptible to constant changes as it depends on the interplay between layer erosion/degradation and mucus secretion (Al-Marhoon et al., 2005; Cone, 2009; Jordan et al., 1998; Newton et al., 1998; Newton et al., 2000). The presence of mucins in gastric fluids results in shear-thinning behaviour of most gastric aspirates. Viscosity of fasted state gastric fluids was reported by Pedersen et al. to range from 1.7 to $9.3 \mathrm{mPa} . \mathrm{s}$ (Table 1). However, as correctly pointed out by the authors, it should be stressed that gastric fluids are highly inhomogeneous, resulting in marked withinsample variation in measured viscosity (Pedersen et al., 2013). Gastric content viscosity typically increases in relation with the ingested meal. As a result, a broad range of viscosity values has been reported by several authors (Table 1). As the meal is emptied from the stomach and water secretion is stimulated, viscosity again decreases (Abrahamsson et al., 2005; Marciani et al., 2000; Radwan et al., 2012).

\subsubsection{Gastric enzymes}

Both pepsin and gastric lipase display pH-dependent behaviour. In case of pepsin, proteolytic activity is maximal at pH 2 but absent at $\mathrm{pH} \geq 5.5$ (Johnston et al., 2007; Piper and Fenton, 1965). As reported earlier, pepsinogen secretion is stimulated by the intake of food (cfr. 3.2. Gastric secretions). Kalantzi et al. corroborated this observation by measuring active pepsin concentrations in both fasted $(250 \mathrm{~mL}$ water) and fed subjects (liquid meal, Ensure Plus ${ }^{\circledR}$ ). The authors reported a significant two-fold increase in median active pepsin concentrations, measured over a one-hour period $\left(0.11-0.22 \mathrm{mg} \cdot \mathrm{mL}^{-1} \mathrm{vs} .0 .26\right.$ - $0.58 \mathrm{mg} \cdot \mathrm{mL}^{-1}$, respectively; Table 1) (Kalantzi et al., 2006).

Gastric lipase is active within a broad range ( $\mathrm{pH} 2$ - 7), with maximal activity between $\mathrm{pH} 3.0$ and 5.5. Considering median $\mathrm{pH}$ values reported in literature, only limited digestion of lipid components is therefore expected to take place in fasted subjects. However, due to sporadic rises in gastric $\mathrm{pH}$ ( $c f r$. 3.3.2. Acidity of gastric content), the extent of gastric lipolytic activity may vary substantially under 
fasted state conditions (Bakala-N'Goma et al., 2015; Pedersen et al., 2013; Ville et al., 2002). Data available in literature focus on concentrations and activity of lipolytic enzymes in fed subjects. However, due to the large variability in methodology, between-study comparison of data proves to be challenging (Armand, 2007). In a series of articles by Carrière and coworkers, total and active secreted amounts of gastric lipase in response to both liquid and mixed solid/liquid meals were determined in gastric aspirates of healthy volunteers. The authors observed similar amounts of active enzyme compared to the total amount determined in the same meal, both for the liquid (14.5 $\pm 10.8 \mathrm{mg}$ vs. $21.6 \pm 14.5 \mathrm{mg}$ ) and mixed solid/liquid meal $(13.2 \pm 4.6 \mathrm{mg}$ vs. $15.2 \pm 5.1 \mathrm{mg})$ (Table 1). These results indicate that most of the gastric lipase present will be active postprandially (Carriere et al., 2005; Carriere et al., 2001; Renou et al., 2001). Triglyceride digestion in the stomach results in the formation of diglycerides, free fatty acids and, to a lesser extent, monoglycerides (Armand et al., 1996a; Armand et al., 1999; Sternby et al., 2002). The contribution of gastric lipase to the overall digestion of lipids throughout the GI tract ranges from 6 - 24\% (Armand et al., 1996a; Borgstrom et al., 1957; Carriere et al., 2005; Carriere et al., 2001; Renou et al., 2001). Factors such as triglyceride content of the meal, meal properties (liquid vs. solid meal) and initial fat droplet size have been reported to play an important role in the extent of triglyceride digestion in the stomach (Armand et al., 1999; Borgstrom et al., 1957; Carriere et al., 2005; Carriere et al., 2000; Carriere et al., 2001; Renou et al., 2001).

\subsubsection{Surface tension, buffer capacity and osmolality}

Water content, the presence of pepsin and sporadic reflux of bile in the stomach are important factors determining surface tension of human gastric aspirates. In a characterization study by Pedersen et al., a mean surface tension value of $34.8 \pm 5.2 \mathrm{mN} . \mathrm{m}^{-1}$ was determined in gastric aspirates of healthy subjects after an overnight fast (Pedersen et al., 2013). Similarly, Efentakis et al. reported surface tension values within a range of $35-45 \mathrm{mN} \cdot \mathrm{m}^{-1}$ (Table 1) (Efentakis and Dressman, 1998). In the first hour after drinking $250 \mathrm{~mL}$ of water, Kalantzi et al. observed a rise in surface tension of fasted state gastric aspirates (median range: $41.9-45.7 \mathrm{mN} . \mathrm{m}^{-1}$ ) compared to previous studies in which no water was consumed, illustrating the influence of gastric water content on surface tension. The authors repeated the study with the same subjects, this time sampling gastric content after ingestion of a liquid meal (500 
mL Ensure Plus ${ }^{\circledR}$ ). The presence of surface-lowering components in the meal (e.g. (phospho)lipids) reduced surface tension by $30 \%$ compared to fasted state results (median range: $30-31 \mathrm{mN} . \mathrm{m}^{-1}$; Table 1) (Kalantzi et al., 2006).

Without prior water ingestion, mean buffer capacity of gastric aspirates amounted to $14.3 \pm 9.5$ mmol..${ }^{1} . \Delta \mathrm{pH}^{-1}$ (Table 1) (Pedersen et al., 2013). However, several publications have reported on buffer capacity of gastric aspirates after the ingestion of water. Due to the fact that (i) buffer capacity of water is very low, and (ii) the ingested volume is usually relatively high compared to the residual gastric volume, buffer capacity is initially decreased after drinking a glass of water. Subsequently, as water empties from the stomach, buffer capacity again increases (Table 1) (Kalantzi et al., 2006; Litou et al., 2016). For instance, Litou et al. reported a six-fold increase in buffer capacity 35 minutes after water ingestion compared to the measured buffer capacity after 10 minutes $\left(27.6 \pm 15.7\right.$ vs. $4.7 \pm 4.6$ mmol.L $\mathrm{L}^{-1} . \Delta \mathrm{pH}^{-1}$, respectively) (Litou et al., 2016).

Osmolality of gastric fluids is mainly determined by the presence of electrolytes in the gastric lumen $\left(\mathrm{Cl}^{-}, \mathrm{Na}^{+}, \mathrm{K}^{+}, \mathrm{Ca}^{2+}\right.$; Table 1). In fasted state, Pedersen et al. and Lindahl et al. determined the mean osmolality of gastric aspirates to be $220 \pm 58$ and $191 \pm 36 \mathrm{mOsm} \cdot \mathrm{kg}^{-1}$, respectively (Lindahl et al., 1997; Pedersen et al., 2013). Given the diluting effect of water, osmolality of gastric aspirates was found to be markedly decreased after ingestion of water (Table 1) (Kalantzi et al., 2006; Litou et al., 2016). As is the case for many gastric fluid characteristics (e.g. $\mathrm{pH}$, viscosity), buffer capacity as well as osmolality measured in gastric samples after intake of a meal will initially be close to that of the ingested meal, followed by a gradual return towards baseline fasted values (Table 1) (Diakidou et al., 2009; Kalantzi et al., 2006).

\subsubsection{Duodenogastric reflux}

Retrograde flow of bile from the duodenum to the stomach has been shown to occur in healthy subjects. Bile salts and phospholipids present in bile may thus enter the stomach and influence gastric fluid characteristics. For instance, gastric $\mathrm{pH}$, surface tension and lipid emulsification processes are susceptible to the presence of alkaline, surface-lowering and solubilizing agents such as bile salts. 
Duodenogastric reflux is of sporadic nature, explaining large variability in reported bile salt concentrations (range reported means: 0.029 - $0.83 \mathrm{mM}$; Table 1) (Di Ciaula et al., 2012; Efentakis and Dressman, 1998; Lu et al., 2010; Mudie et al., 2014; Schiller et al., 2005). Although not harmful if occasionally occurring, frequent duodenogastric reflux events may result in the development of several pathologies (e.g. ulcers, inflammation of stomach and/or esophagus) (Chen et al., 2013; Dai et al., 2002; Dewar et al., 1982).

\subsubsection{Population-related differences in gastric fluid characteristics}

The stomach is a complex, dynamic environment constantly changing its characteristics in response to exogenous and endogenous stimuli. Therefore, physicochemical characteristics of gastric fluids may significantly vary among populations (Fig. 3).

\subsubsection{Age-neonates, infants and children}

As a result of the ongoing changes in the human body after birth, composition of gastric fluids can be presumed to significantly change during this maturation process. However, literature on gastric fluid composition in healthy neonates and children is scarce, due to ethical considerations. Therefore, publications on this topic sometimes include ill children, for which an effect of a certain pathology on the parameter studied can often not be ruled out. Nevertheless, some trends regarding changes in gastric secretory function with age have been reported. For instance, residual gastric volume is thought to be prone to changes during early life. Comparing literature data between age groups, Bourlieu et al. reported markedly lower residual volumes in neonates ( $<1$ day) and infants (age range not reported) (mean volume: 2 - 2.65 and $2.4 \mathrm{~mL}$, respectively) compared to reported volumes in adults (cfr. 3.3.1. Gastric volume) (Bourlieu et al., 2014). These observations indicate that important differences in overall secretory output may indeed exist between age groups.

Reports on age-dependent differences more specifically mention changes in gastric acid secretion and pepsin output, with implications for the acidity and proteolytic activity of gastric contents. Acidity of gastric contents is thought to fluctuate markedly within the first few days after birth (Debotton and Dahan, 2014; Seyberth and Kauffman, 2011). Data from studies with neonates performed in the 1940s, 
50s and 60s indicate that at birth, gastric $\mathrm{pH}$ is close to neutral. This observation has been suggested to be related to the swallowing of amniotic fluid, as the acidity of gastric contents increases towards $\mathrm{pH}$ $\sim 3$ within 24 hours (Avery et al., 1966; Ebers et al., 1956). Subsequently, gastric acidity decreases during the next ten days after which gastric fluids re-acidify gradually (Miller, 1941). Gastric $\mathrm{pH}$ is thought to reach adult values within a two year period (Bourlieu et al., 2014; Fallingborg et al., 1990; Kaye, 2011; Schmidt et al., 2015; Yu et al., 2014).

With regard to pepsin, several authors reported lower pepsin concentrations in children compared to adults under both fasted and fed state conditions (Armand et al., 1996b; Gharpure et al., 2000; Westhus, 2004). Mean postprandial pepsin output, for instance, appeared to be lower in preterm infants (age range: 1 - 11 weeks; mean age: 5 - 6 weeks) compared to healthy adults (Armand et al., 1996b). However, the duration of this maturation period is less well established. DiPalma et al. observed similar fasted pepsin activity regardless of age (age range: 3 months - 26 years), indicating that, from three months onward, pepsin output reaches adult levels (DiPalma et al., 1991). However, in studies performed by Gharpure et al. and by Westhus et al., markedly different mean pepsin concentrations in children $<1$ year old compared to older children were reported (Gharpure et al., 2000; Westhus, 2004). Unfortunately, neither of these studies did include patient demographics. Contrary to pepsin, no differences regarding both fasted and fed state gastric lipase activity were observed between children and adults, suggesting full lipid-digesting capacity directly from birth (Armand et al., 1996b; DiPalma et al., 1991).

\subsubsection{Age-elderly}

Feldman et al. investigated the influence of age on basal and peak gastric acid secretion. Although basal secretory output was not affected by age, the authors reported a significant decrease in mean peak gastric acid output (PAO) in elderly (> 65 years) compared to young (18-34 years) and middle-aged subgroups (35-65 years) $\left(19.0 \pm 13.2\right.$ vs. $29.3 \pm 12.7$ vs. $29.9 \pm 14.1$ mmol. $\mathrm{h}^{-1}$, respectively). Morphological examination of gastric biopsy samples from the participants revealed that gastritis, in particular atrophic gastritis, was more prevalent in the elderly population. Approximately a five-fold decrease in mean PAO was observed in subjects displaying atrophic changes to gastric mucosa compared to subjects with 
normal histology ( $6.8 \pm 10.7$ vs. $32.3 \pm 12.0 \mathrm{mmol}^{-1} \mathrm{~h}^{-1}$, respectively). These atrophic changes, rather than age itself, are therefore most likely responsible for the observed decrease in PAO in the elderly population (Feldman et al., 1996). Several other authors corroborated these findings, providing a strong case regarding the lack of age-specific changes in gastric acid secretion in adults (Alvis and Hughes, 2015; Haruma et al., 2000; Katelaris et al., 1993; Kekki et al., 1982; Shih et al., 2003). Similar observations could be made with regard to mucus layer thickness. Although no age-related changes in mucus layer thickness were observed in $H$. pylori negative subjects, mucus layer thickness was found to significantly decrease with age in H. pylori positive subjects (Newton et al., 2000). Again, it may be hypothesized that this change might be due to a higher prevalence of gastric atrophy and gastritis in elderly subjects as a result of long-time infection with H. pylori (Al-Marhoon et al., 2005; Newton et al., 1998; Newton et al., 2000). Lastly, Feldman et al. investigated changes in basal and peak pepsin output (BPO/PPO) in relation to age. Both BPO and PPO were significantly lower in the elderly population. However, unlike for gastric acid secretion and mucus layer thickness, this observation was found to be irrespective of the presence of an underlying pathology, indicating that old age itself decreases pepsin output (Feldman et al., 1996).

\subsubsection{Gender and race}

Numerous studies investigating gender differences with respect to gastric acidity and acid secretion have been published. Several of these studies reported decreased gastric acid output in women compared to men (Baron, 1969; Kekki et al., 1982; Prewett et al., 1991). For example, Prewett et al. observed significantly lower exposure of the stomach to gastric acid in women compared to men, measured over a 24 hour period (median: 485 mmol.h.L ${ }^{-1}$ vs. 842 mmol.h.L ${ }^{-1}$, respectively) (Prewett et al., 1991). These findings apply to both pre- and postprandial acid content, as subjects were served three meals (breakfast, lunch and dinner) during the observation period. Remarkably, Feldman et al. and Prewett et al. reported consistently higher plasma gastrin concentrations in females compared to males. These results appear counter-intuitive, as gastrin is an important stimulant of gastric acid secretion. To explain the lower acid output in women despite higher plasma gastrin concentrations, several authors attributed this observation to a relative decrease in sensitivity of parietal cells to gastrin as well as a lower parietal cell 
mass in females compared to males (Feldman et al., 1983; Kekki et al., 1982; Prewett et al., 1991). More recent studies, however, indicate that gender does not affect gastric acidity. This is illustrated in a retrospective study by Shih et al., reporting similar mean gastric $\mathrm{pH}$ in men compared to women (3.01 \pm 1.42 vs. $2.96 \pm 1.36$, respectively) (Shih et al., 2003). It should be mentioned, however, that medical history of these subjects was not taken into account. Nevertheless, reports by Lindahl et al. and Dressman et al. corroborate the statement that gastric content acidity does not differ between both sexes (Dressman et al., 1990; Lindahl et al., 1997). Given the conflicting findings published so far, an unambiguous statement on the influence of gender on intragastric acidity does not seem justified.

In a study by Lindahl et al., characterizing aspirated gastric fluids from healthy volunteers, no genderrelated differences in electrolyte concentrations, osmolality, bile salt concentrations and protein content between men and women were observed (Lindahl et al., 1997). Furthermore, Al-Marhoon et al. reported similar mean mucus layer thickness when comparing men to women $(55.5 \pm 1.1$ vs. $48.2 \pm 1.1 \mu \mathrm{m}$, respectively) (Al-Marhoon et al., 2005). Although literature reports on gender-related differences remain scarce, this study suggests that gender most likely does not influence mucus layer thickness.

Basal and maximal acid output were reported to be lower in a Japanese population compared with American and Finnish study populations (Feldman et al., 1996; Haruma et al., 2000; Katelaris et al., 1993). The authors linked this finding to the increased prevalence of fundic atrophic gastritis in the Japanese study population. Similar to age-related differences, it therefore seems that racial differences in gastric acid secretion can be attributed to a different prevalence of atrophic changes to gastric mucosa between populations (Shih et al., 2003). A literature review by Tonkic et al. on the worldwide epidemiology of $H$. pylori infections, linked to the development of atrophic gastritis, further indicates that prevalence may indeed differ substantially between countries (Tonkic et al., 2012).

\subsubsection{Patient populations}

Pathological conditions may have a significant impact on gastric volume due to (i) alterations to the gastric mucosa, hereby impairing gastric secretion or (ii) excessive stimulation of the stomach's secretory function. For instance, mean secretory volume has been found to be significantly lower upon 
stimulation with pentagastrin in patients with gastric atrophy compared to healthy subjects $(188 \pm 26$ $\mathrm{mL}$ vs. $261 \pm 15 \mathrm{~mL}$, respectively) due to chronic inflammation of gastric mucosa (Katelaris et al., 1993). In the case of patients suffering from Zollinger-Ellison Syndrome (ZES), excessive stimulation of gastric acid secretion, rather than changes to the gastric mucosa, may cause significant changes in gastric content volume. In a study by Dubois et al., mean fasting gastric volume increased almost twofold in ZES patients compared to healthy controls ( $42 \pm 6 \mathrm{~mL}$ vs. $24 \pm 5 \mathrm{~mL}$, respectively) (Dubois et al., 1977). However, more patients should be included in these investigations in order to make a firm statement on the effect of ZES on gastric volume.

Mucus layer integrity can be considerably compromised in several disease states; however, it is not always clear whether impaired integrity is either the result or the cause of the disease (Ensign et al., 2012). Although the mucus layer is an effective barrier in protecting gastric mucosa from bacterial infections, $H$. pylori is capable of colonizing the stomach most likely by causing local increases in gastric acidity (Celli et al., 2009). In general, mean mucus layer thickness was found not to be affected by $H$. pylori infection, as reported by Al-Marhoon et al. $(51.3 \pm 1.1 \mu \mathrm{m}$ vs. $48.8 \pm 1.1 \mu \mathrm{m}$ in infected vs. noninfected patients, respectively) and Newton et al. (104 $\pm 26 \mu \mathrm{m}$ vs. $106 \pm 30 \mu \mathrm{m}$, respectively) (AlMarhoon et al., 2005; Newton et al., 1998). However, when only taking into account $H$. pylori positive subjects with marked inflammation of gastric mucosa (gastritis or gastric atrophy), Newton et al. found mucus layer thickness to be significantly decreased ( $84 \pm 13 \mu \mathrm{m})$ (Newton et al., 1998). These results indicate that not $H$. pylori infection as such but rather the severity of the infection may affect mucus layer thickness. With regard to mucus layer composition, Newton et al. reported a significantly lower mean percentage of mucin in the gastric mucus layer of $H$. pylori infected subjects compared to $H$. pylori negative, age-matched controls (60 $\pm 16 \%$ vs. $72 \pm 12 \%$, respectively) (Newton et al., 1998). In a metaanalysis performed by Niv et al., expression of the main protecting mucin (i.e. MUC5AC) was found to be significantly decreased in $H$. pylori infected subjects (Odds Ratio (OR): 0.447; p: 0.036). In contrast, mucin 6 (MUC6)-expression, with antibiotic properties, was increased (OR: 9.244; p: 0.014) indicating that changes in mucin expression levels are most probably subtype-specific (Niv, 2015). These 
observations indicate that mucus layer structure, and subsequently layer strength, may be altered due to H. pylori infection while layer thickness remains generally unaffected.

Gastric lipase secretion and activity is markedly increased in patients with severely hampered pancreatic secretory function, probably as a compensatory mechanism to (partly) restore intestinal uptake of triglyceride digestion products. Balasubramanian et al. reported two-fold higher postprandial gastric lipase activity in cystic fibrosis patients with pancreatic steatorrhea compared to age- and sex-matched controls (mean: $596 \pm 25 \mathrm{nmol} \cdot \mathrm{mL}^{-1} \cdot \mathrm{min}^{-1}$ vs. $299 \pm 28 \mathrm{nmol} \cdot \mathrm{mL}^{-1} \cdot \mathrm{min}^{-1}$, respectively) (Balasubramanian et al., 1992). Similarly, Carrière et al. observed an extensive increase in mean gastric lipase output in patients with severe chronic pancreatitis compared to healthy subjects $(48.6 \pm 31 \mathrm{mg}$ vs. $14.7 \pm 8.7 \mathrm{mg}$, respectively) (Carriere et al., 2005).

\subsection{Gastric motility and gastric emptying}

\subsubsection{Gastric motility}

Musculature of the stomach is characterized by the presence of both circular and longitudinal layers of muscularis (Soybel, 2005; Trowers and Tischler, 2014a). This anatomical feature results in distinct differences in regional contributions to gastric motility. The proximal stomach (fundus - upper corpus region) is crucial for the accommodation of food boluses by acting as a distensible reservoir through the regulation of gastric wall tonicity, whereas the distal stomach (midcorpus - antrum region) controls mixing, propulsion and eventually expulsion of gastric contents from the stomach (Hasler, 2009; O'Connor and O'Morain, 2014; Trowers and Tischler, 2014a). Distal gastric motility is regulated by a network of gastric pacemaker cells, i.e. 'Interstitial cells of Cajal (ICC)' spread along the gastric wall. Via coupling to gastric smooth muscle cells, the ICC network induces the propagation of peristaltic slow waves originating from the region between upper and midcorpus towards the pylorus (Fig. 4). Although slow wave activity is continuously present, this does not imply continuous contractions of the distal stomach; an additional excitatory stimulus (neural, hormonal, pharmacological) is required on top of slow wave propagation in order to induce a gastric contraction (Deloose et al., 2012; Hasler, 2009; 
Morgan and Szurszewski, 1980; O'Connor and O'Morain, 2014; Sanders et al., 2006). In man, slow wave frequency has been documented to be approximately $3 \cdot \mathrm{min}^{-1}$, increasing in amplitude and propagation velocity towards the pylorus (Hasler, 2009; Marciani et al., 2001a; O'Grady et al., 2010).

Distal gastric motility is characterized by distinct contractile patterns under both fasted and fed state conditions. Gastric barostat and High-Resolution Manometry (HRM) techniques can be used to study gastric motor function, mainly for research purposes but also for clinical purposes (Camilleri et al., 2008; Deloose et al., 2012; Desipio et al., 2007; Dooley et al., 1992; J. van der Schaar P., 1999; Verhagen et al., 1998). The barostat device uses an inflatable bag which is positioned in the fundus to study gastric pressure/volume events in response to a meal or other stimuli and to perform gastric distensions to study gastric accommodation and sensitivity. The HRM technique, utilizing either water-perfused or solid-state pressure sensors, has been extensively used to monitor motor function in the esophagus, but has now been shown to be a non-invasive alternative to the barostat to study gastric accommodation (Bredenoord and Smout, 2008; Distrutti et al., 1999; Janssen et al., 2011; Notivol et al., 1995; Tack et al., 1998).

Between meals, three cyclically recurring phases in the motor pattern of the GI tract can easily be distinguished based on contraction frequency and amplitude (Fig. 4). This so-called '(Interdigestive) Migrating Motor Complex ((I)MMC)' was first described in man by Vantrappen et al. in 1977 and has been the subject of extensive research ever since (Vantrappen et al., 1977). Phase I of the MMC is a phase of contractile quiescence, characterized by the absence of gastric contractions. Phase I is followed by a period of irregular phase II contractions. In a study with 40 healthy volunteers, Bortolotti et al. reported a mean antral contraction amplitude of $39.7 \pm 14.4 \mathrm{mmHg}$ during phase II of the MMC (Bortolotti et al., 2000). In their study investigating gastrointestinal sounds, Tomomasa et al. observed a correlation of gastrointestinal rumbling with late-stage phase II contractions (Tomomasa et al., 1999). Phase III, the final phase of the cycle, can originate either in the stomach or the duodenum (Luiking et al., 1998). A gastric phase III is characterized by a strong burst of contractions for 2-6 minutes at a constant rate of 2-3 contractions. $\mathrm{min}^{-1}$ and is often referred to as the 'gastric housekeeper wave' 
(Cassilly et al., 2008; Conklin, 2009). Bortolotti et al. reported a mean contraction amplitude of $88 \pm$ $31.7 \mathrm{mmHg}$ (Bortolotti et al., 2000). However, antral contractility spikes up to $200 \mathrm{mmHg}$ have been reported in human subjects in association with phase III of the MMC (Cassilly et al., 2008). Although undoubtedly influenced by differences in study design and measuring technique used, the wide range of reported values is thought to reflect large inter-individual variability in contractile strength.

Mean duration of one MMC cycle in healthy human subjects has been reported to be between 96 and 172 minutes (Bortolotti et al., 2000; Dalenback et al., 1993; Dooley et al., 1992; Luiking et al., 1998; Oberle et al., 1990; Pieramico et al., 1995), indicating that cycle length as well is highly variable. Dooley et al. published data specifically on variation in MMC cycle length. Apart from marked inter-individual differences (mean: $172 \pm 72 \mathrm{~min}$; range: 112 - $230 \mathrm{~min}$ ), intra-individual variation has been reported to be highly pronounced. The authors observed variations in overall MMC cycle length up to $88 \%$ when assessed in the same subjects on separate days (Dooley et al., 1992). A link between temporal variations in gastric secretion rate on the one hand and MMC cycli on the other hand has been reported by several authors. Vantrappen et al. reported a significantly higher output of gastric acid and pepsin in the $30 \mathrm{~min}$ period prior to phase III contractions in the duodenum, corresponding roughly to late phase II/phase III contractions in the stomach (Vantrappen et al., 1979). Similarly, Dalenbäck et al. observed changes in acid and bicarbonate secretion in healthy volunteers during late phase II/phase III of the MMC, resulting in acidification of gastric contents during phase III followed by alkalinisation during phase I (Dalenback et al., 1993).

After meal intake, the proximal stomach serves as a reservoir for the accommodation of ingested food prior to its propagation to the distal stomach and duodenum. Via tonic contraction/relaxation of the proximal gastric wall, the stomach is able to accommodate large boluses of food with little changes in intragastric pressure (Hasler, 2009). Ingestion of food causes immediate cessation of interdigestive gastrointestinal motility in the distal stomach (Vantrappen et al., 1977). Fed state contractile patterns are characterized by antral contraction waves, originating in the midcorpus and propagating towards the pylorus at a mean frequency of $3.0 \pm 0.2 \cdot \mathrm{min}^{-1}$ (i.e. slow wave frequency) irrespective of meal consistency (Marciani et al., 2001a; Marciani et al., 2001b). Contraction waves increase in strength as 
they move along the gastric wall, causing partial occlusion of the antral lumen (Kwiatek et al., 2006; Marciani et al., 2001b; Schulze, 2006; Trowers and Tischler, 2014a). Bortolotti et al. reported a mean contraction strength of $51.7 \pm 12.4 \mathrm{mmHg}$ in fed subjects, corresponding to phase II-like contraction strength (Fig. 4) (Bortolotti et al., 2000). Koziolek et al. recorded marked high-pressure events and highly variable pressures on a solid motility capsule (SmartPill ${ }^{\circledR}$, Given Imaging, Israel) in the fed stomach, which may be related to the position of the capsule in the stomach (antrum vs. corpus) and/or inter-individual variability (Koziolek et al., 2015c). As fluid dynamics are hard to visualize in an in vivo setting, attempts have been made to gain insight into fluid transport within the fed stomach using computational models based on gastric physiology data available in literature. These models predicted the establishment of retropulsive fluid motions toward the proximal stomach as a result of only partial occlusion of the antral lumen during contraction waves. Furthermore, circular motions ('eddies') inbetween antral contraction waves are thought to contribute to the mixing of a food bolus with gastric secretions ('chyme') (Ferrua and Singh, 2010; Pal et al., 2004). Besides facilitating chemical digestion of a meal by ensuring adequate mixing with gastric acid and enzymes, the stomach also mechanically breaks down solid food particles (Conklin, 2009; Trowers and Tischler, 2014a). Food particle size reduction results from the well-coordinated action of distal motor function and pyloric activity. As a contraction wave reaches the midantrum, the pylorus is in an open configuration allowing the emptying of chyme. By the time the contraction wave arrives at the distal antrum, this configuration changes to a closed position causing the food bolus dragged along by the contraction wave to crash against the closed pylorus, hereby facilitating grinding of large particles (Hasler, 2009; Indireshkumar et al., 2000; O'Connor and O'Morain, 2014).

\subsubsection{Gastric emptying}

Transfer of gastric contents from the stomach to the duodenum is closely linked to contractility of the gastric wall. As gastric motility varies significantly depending on prandial state, gastric emptying of non-caloric and caloric contents is distinctly different. Secondly, distinction should be made between the emptying of liquid and solid gastric content. 
In fasted subjects, emptying of both liquid and solid gastric contents in the duodenum is closely linked to the peristaltic, phasic contractions of the MMC cycle. In a transpyloric flow study, liquid gastric contents were observed to be transferred from stomach to duodenum at all times during the MMC cycle. The authors reported fluid flow rates to be lowest during MMC phase I and highest during MMC phase III (Savoye et al., 2003). These findings confirm earlier observations by Oberle et al., who reported an increase in liquid gastric emptying rate with increasing gastric contractility (GE $t_{1 / 2}$ phase $\mathrm{I}>\mathrm{GE} \mathrm{t}_{1 / 2}$ phase II > GE $t_{1 / 2}$ phase III); the increase was most pronounced in the case of relatively small ingested volumes (Oberle et al., 1990). Emptying of non-caloric liquids is characterized by first order kinetics: emptying is initially rapid and subsequently slows down at a later stage (Gonzalez et al., 2000; Hasler, 2009; Siegel et al., 1988). Non-caloric solids (e.g. tablets, capsules) as well as indigestible food remnants are cleared from the stomach by means of peristaltic contractions. Wireless motility capsule studies have indicated that in order to transfer large, solid objects from the stomach to the duodenum, strong phase III contractions are generally required (Smartpill ${ }^{\circledR}$; capsule dimensions: 26 x $13 \mathrm{~mm}$ ) (Koziolek et al., 2015c). Illustrating the motility-dependency of large, solid objects with regard to emptying, Cassilly et al. observed a good correlation between the emptying of a Smartpill ${ }^{\circledR}$ and the occurrence of gastric phase III contractions (r: 0.81) (Cassilly et al., 2008).

The rate at which caloric meals empty from the stomach is regulated by numerous factors. The small intestine (duodenum, jejunum and ileum) intensively generates feedback signals in response to foodrelated stimuli. Changes in duodenal $\mathrm{pH}$, nutrient load and osmolality have been shown to trigger feedback mechanisms in the small intestine, slowing down gastric emptying (Chaw et al., 2001; Heddle et al., 1989; Holgate and Read, 1985; Meeroff et al., 1975; Van Citters and Lin, 1999).

Apart from regulation of gastric emptying via intestinal feedback mechanisms, gastric emptying of caloric meals is to a large extent dictated by the specific properties of the meal in question. Meal structure, for instance, plays an important role in the gastric emptying of food. Distinction has to be made between emptying of liquid and solid meal components, respectively. Caloric solids display a biphasic emptying behaviour: meal emptying is preceded by a lag phase during which no emptying of the meal from the stomach occurs (Camilleri et al., 1985; Collins et al., 1996; Horowitz et al., 1984). 
This lag phase is considered to be the time needed for the stomach to adequately process the ingested solids. Siegel et al. observed that the lag phase $\left(\mathrm{t}_{\mathrm{lag}}\right)$, and consequently GE $\mathrm{t}_{1 / 2}$, were considerably shorter for a semisolid meal (egg-white sandwich) than for an equicaloric solid meal (chicken liver) with similar fat, carbohydrate and protein content (mean $\mathrm{t}_{\mathrm{lag}}: 31 \pm 2 \mathrm{~min}$ vs. $62 \pm 5 \mathrm{~min}$, respectively) (Siegel et al., 1988). Camilleri et al reported an inverse correlation between lag phase duration and antral motility (Camilleri et al., 1985). A similar delay in onset of gastric emptying is typically not observed for caloric liquids (Gonzalez et al., 2000; Hasler, 2009; Horowitz et al., 1984; Hutson et al., 1989). This may be explained by the intragastric location of liquid meals after ingestion. Imaging studies demonstrated rapid dispersal of liquids throughout the entire stomach, whereas solids are retained in the proximal stomach for a longer period of time (Collins et al., 1996; Sachdeva et al., 2013). As meal processing prior to emptying is not required for liquid meals, gastric emptying can be readily initiated, explaining the general absence of a lag period for these types of meals. Ingestion of mixed solid/liquid meals has been proposed to delay gastric emptying of the liquid component due to increased proximal gastric retention of the liquid meal (Collins et al., 1996). In a comprehensive study by Kwiatek et al., an increase in liquid meal volume was observed to result in faster gastric emptying rates (Kwiatek et al., 2009). Furthermore, caloric load has been reported to significantly affect gastric emptying behaviour. Using liquid meals, several studies have demonstrated a delay in the emptying of meals with increased caloric density (Calbet and MacLean, 1997; Gonzalez et al., 2000; Kwiatek et al., 2009; Vist and Maughan, 1995). Furthermore, meal composition has been proposed to influence emptying behaviour. For instance, high fat content of a meal has been reported to decrease gastric emptying rate, possibly via inducing changes in (i) meal distribution in the stomach and/or (ii) intragastric hydrodynamics (Boulby et al., 1999; Houghton et al., 1990; Nakae et al., 1999). These hypotheses may explain the lag phase in the gastric emptying of a high-fat, high-caloric liquid meal (Ensure Plus ${ }^{\circledR}$; fat content: 28\%) observed by Sachdeva et al. (Sachdeva et al., 2013).

\subsubsection{Population-related differences in gastric motility}

\subsubsection{Age-neonates, infants and children}


Literature reports suggest marked changes in gastric motor function during early life (Fig. 3). Chen et al. reported significant differences in gastric slow wave activity between age groups. Slow wave activity in neonates ( 1 - 2 days) and infants (2 - 6 months) differed markedly compared to children (4 - 11 years), as measured by the percentage of regular slow wave patterns $\left(2-4\right.$ waves. $\left.\mathrm{min}^{-1}\right)$ recorded during the experiment $(26.6 \pm 3.3 \%$ vs. $70.0 \pm 6.1 \%$ vs. $84.6 \pm 3.2 \%$, respectively). These results suggest a maturation of gastric motor function during the first few months after birth, reaching adult characteristics at childhood (\% regular slow wave patterns: $84.6 \pm 3.2 \%$ in children vs. $88.9 \pm 2.2 \%$ in adults). No difference between preterm neonate's and term neonate's slow wave activity was observed in this study ( $30.0 \pm 4 \%$ vs. $26.6 \pm 3.3 \%$, respectively) (Chen et al., 1997). These results were later corroborated by Liang et al. and Zhang et al., who performed consecutive electrogastrographic (EGG) measurements in healthy preterm neonates over a six month period. A gradual increase in gastric slow wave activity with age was observed (Liang et al., 1998; Zhang et al., 2006). Furthermore, Liang et al. reported a marked stimulation of slow wave activity after enteral feeding during the first two months, which is absent during later stages of life, again suggesting maturation of gastric motor function during early life (Liang et al., 1998).

Observed differences in gastric motor function in neonates and infants may lead to differences in gastric emptying behaviour compared to 'normal' gastric emptying in adults (Fig. 3). Gupta et al. investigated gastric retention of an infused glucose solution in $1-2$ day old neonates during the first 36 hours after birth. Gastric retention was found to be increased in neonates 4 - 12 hours after birth compared to 22 36 hours after birth. Furthermore, gestational age significantly affected gastric retention of the glucose solution during the first few hours as demonstrated by the larger percentage of retention at $4-12$ hours in preterm neonates compared to term neonates ( $63 \pm 2.4 \%$ vs. $48 \pm 2.3 \%$, respectively) (Gupta and Brans, 1978). Complementary, Blumenthal et al. investigated the pattern of gastric emptying in neonates (5 - 26 days). Gastric emptying patterns after infusion of a dextrose solution were similar to gastric emptying of liquids in adults (cfr. 3.4.2. Gastric emptying) (Blumenthal and Pildes, 1979). However, as gastric emptying of liquids is mainly driven by the fundus, these observations cannot be generalized to the emptying of solids in neonates by gastric peristaltic contractions. Using ultrasonography, Carlos et 
al. reported a delay in gastric antral distension in very preterm neonates (gestational age: 23 - 29 weeks) after enteral feeding; suggesting slower gastric emptying of orally administered solid dosage forms initially after birth (Carlos et al., 1997). With regard to drug pharmacokinetics, alterations in gastric emptying may result in variable systemic drug exposure compared to adults. For instance, a marked delay in absorption of acetaminophen (paracetamol, formulated as an elixir) has been reported for neonates during the first days of life (Anderson et al., 2002). As paracetamol is a BCS Class I compound, gastric emptying can be assumed to be the rate-limiting step for intestinal absorption. Fallingborg et al. reported ranges in gastric residence time of a telemetric capsule in healthy children ( 8 - 14 years) similar to ranges observed by Koziolek et al in healthy adult volunteers ( $0.2-2.3$ vs. 0.12 - $3.36 \mathrm{~h}$, respectively); indicating that maturation of gastric motor function is most likely completed at childhood (Fallingborg et al., 1990; Koziolek et al., 2015b).

\subsubsection{Age-elderly}

At present, data on gastric emptying in elderly are quite inconsistent. While some authors report significant changes in emptying behaviour in an older population compared to younger subjects, others report only a difference in emptying of specific meal components or no difference at all. Investigating gastric emptying of both a solid (chicken liver) and liquid meal (glucose solution) using gammascintigraphy, Horowitz et al. observed a significant delay in emptying of both meal components in elderly (age range: 70 - 84 years) compared to a younger population (age range: 21 - 62 years). Mean gastric emptying half-time of the solid meal amounted to $103 \pm 8$ minutes in elderly versus $78 \pm 4$ minutes in the younger population, while half of the liquid meal was emptied within $25 \pm 3$ minutes in elderly versus $19 \pm 1$ minutes in the younger population (Horowitz et al., 1984). These observations were later corroborated by Clarkston et al, who reported a similar delay in gastric emptying with age for both solid (GE t $\mathrm{t}_{1 / 2}: 127 \pm 13 \mathrm{~min}$ in adults, mean age: 30 years vs. $182 \pm 26 \mathrm{~min}$ in elderly, mean age: 76 years) and liquid meal components ( $35 \pm 3$ min vs. $47 \pm 4$ min, respectively) (Clarkston et al., 1997). Moore et al. observed a significant delay in gastric retention of orange juice in elderly compared to young subjects ( $40 \pm 4 \%$ vs. $25 \pm 4.7 \%$ retention after $120 \mathrm{~min}$, respectively); however, mean gastric emptying half-time was not significantly different between both populations ( $94 \pm 13$ vs. $68 \pm 7.4$ min, 
respectively). In the same study, no effect of age on the emptying of a solid meal was observed (GE $t_{1 / 2}$ : $104 \pm 10 \mathrm{~min}$ in a younger population versus $105 \pm 17 \mathrm{~min}$ in elderly) (Moore et al., 1983). Using Doppler ultrasonography, O'Donovan et al. assessed changes in the emptying of a glucose solution with age. The solution emptied faster from the stomach in younger subjects (mean age: 24.5 years) compared to elderly subjects (mean age: 73.5 years) due to the prolonged retention of the solution in the proximal stomach in elderly subjects (O'Donovan et al., 2005).

Contrary to the earlier discussed publications, several authors reported that gastric emptying behaviour is preserved at later stages of life (Madsen and Graff, 2004; Nakae et al., 1999). In a large multicentre study, Tougas et al. even reported more rapid emptying of a low-fat meal in an older population (age range: 50 - 73 years) (Tougas et al., 2000). However, the wider age limits for the population in this study compared to other studies hamper between-study comparison of results. In general, reasons for conflicting results may be multifactorial. For instance, between-study differences in age of both the older subjects and the younger control subjects may lead to mixed results. Secondly, the thoroughness of the physical examination prior to inclusion in the study can often not be inferred from the publication. As the risk of an underlying pathology increases with age, these pathologies might influence gastric emptying and thus study outcome. Lastly, differences in methodology, study design and parameters measured may lead to contradictory conclusions.

Furthermore, one could question the importance of a generally modest delay/increase in gastric emptying with age. Although in some cases statistically significant findings were reported, such limited effects are unlikely to significantly affect oral drug pharmacokinetics. However, as data on the link between changes in gastric emptying with age and drug pharmacokinetics is lacking, the clinical significance of these findings with regard to drug disposition remains to be elucidated (Clarkston et al., 1997; Horowitz et al., 1984).

\subsubsection{Gender}

The influence of gender on gastric emptying behaviour in humans has received quite some attention in the past, especially during the ' $80 \mathrm{~s}$ and ' $90 \mathrm{~s}$. With regard to the emptying of caloric liquids, mixed 
results have been obtained; while some authors observed a delay in emptying of liquid meals in women, others reported no significant differences in emptying of liquid meal components (Bennink et al., 1998; Caballero-Plasencia et al., 1999; Hutson et al., 1989). This discrepancy may be partly explained by the selection of the liquid component used in the study. For example, due to the high-fat content of the Ensure ${ }^{\circledR}$ liquid meal, gastric emptying is more similar to that of a solid meal (cfr. 3.4.2 Gastric emptying) and thus is likely to differ from that of caloric liquids such as orange juice.

In the case of solid meals, the majority of publications agree that gastric emptying of these meals markedly differs between healthy women and men (Fig. 3). Several authors reported significantly slower emptying of solid food components in women compared to men (Bennink et al., 1998; CaballeroPlasencia et al., 1999; Hermansson and Sivertsson, 1996; Hutson et al., 1989; Tougas et al., 2000). For instance, mean retention percentage of a Swedish pancake meal in the stomach after two hours was observed to be significantly larger in female versus male subjects $(62.8 \pm 6.3 \%$ vs. $43 \pm 14.1 \%$, respectively). Half of the meal emptied considerably faster in men compared to women (GE $\mathrm{t}_{1 / 2}: 111.2$ $\pm 34.5 \mathrm{~min}$ vs. $158.2 \pm 24 \mathrm{~min}$, respectively) (Hermansson and Sivertsson, 1996). As emptying of solid food components is mainly driven by gastric slow wave contractions, it could be hypothesized that these differences in gastric emptying of solids may reflect substantial differences in gastric motility. However, this hypothesis has been rejected by several independent investigations, in which gastric antral motility was observed to be similar in men and women, both in frequency and strength of antral contractions (Bennink et al., 1998; Hutson et al., 1989). Bennink et al. reported longer retention of a solid meal (scrambled eggs with bread) in the proximal part of the stomach in women, suggesting the possibility of delayed initiation of antral grinding of solids and thus an increase in onset of gastric emptying (Bennink et al., 1998). However, this increase in lag time was not observed by Hermansson et al. after a Swedish pancake meal (Hermansson and Sivertsson, 1996). Fluctuations in hormone levels have been suggested to influence gastric emptying behaviour in women (Bennink et al., 1998; Caballero-Plasencia et al., 1999; Hermansson and Sivertsson, 1996). Therefore, multiple studies have included both premenopausal women during different phases of their menstrual cycle and postmenopausal women. While several reports in the past have indicated that menstrual phase does not significantly affect gastric 
emptying behaviour, a more recent publication by Brennan et al. in 2009 again challenges these findings (Brennan et al., 2009; Caballero-Plasencia et al., 1999; Hutson et al., 1989). In light of the data published so far, a definitive statement on the underlying mechanism causing altered gastric emptying of test meals in women compared to men is not possible at present. Although these modest differences between man and women may be relevant from a diagnostic point of view, no data is currently available to judge their relevance for oral drug absorption.

\subsubsection{Patient populations}

Several pathologies have been reported to cause a delayed transfer of contents from the stomach to the duodenum. In a study population of 48 gastro-esophageal reflux disease (GERD) patients, Cunningham et al. reported slower gastric emptying of a solid meal (beef) and a liquid meal (dextrose solution) in $44 \%$ and $17 \%$ of cases, respectively (Cunningham et al., 1991). Similarly, a chicken liver meal emptied significantly slower from the stomach in $33.5 \%$ of patients suffering from functional dyspepsia compared to healthy controls (Stanghellini et al., 1996). Miyaji et al. reported alterations in gastric emptying and gastric motor function in approximately $70 \%$ of non-ulcer dyspepsia patients. $67.4 \%$ of included patients were positive for $H$. pylori, suggesting a possible effect of $H$. pylori on gastric motility. Indeed, after treatment of the infection, gastric emptying normalized in these patients. However, as a proportion of $H$. pylori negative patients also displayed a significant delay in gastric motor function and emptying, H. pylori infection is most likely not the only factor causing deviating gastric motility in these patients (Miyaji et al., 1999). In those cases in which gastric emptying is severely delayed for a longer period of time and not caused by gastrointestinal obstruction, delayed gastric emptying is sometimes referred to as 'gastroparesis'. Using the Smartpill ${ }^{\circledR}$ technology to study GI transit times, Sarosiek et al. reported a significant increase in the capsule's residence time in the stomach of patients suffering from gastroparesis compared to controls (median: $5.4 \mathrm{~h}$ vs. $3.6 \mathrm{~h}$, respectively) as well as in the time needed to pass through the entire GI tract compared to controls (median: $45.9 \mathrm{~h}$ vs. $27.7 \mathrm{~h}$, respectively) 
(Sarosiek et al., 2010). These findings may have important implications for the performance of dosage forms, e.g. time-dependent drug-release strategies.

Often, patients suffering from diabetes mellitus or Parkinson's disease display extensive gastroparesis (Fig. 3) (Heetun and Quigley, 2012; Hermann et al., 1998; Horowitz et al., 1991; Jones et al., 1995; Unger et al., 2010). Heetun et al. bundled relevant data on the effect of Parkinson's disease on gastric emptying in a comprehensive review (Heetun and Quigley, 2012). From their literature search, it can be deduced that gastric emptying in almost all published reports was delayed in a significant proportion of Parkinson disease patients studied. However, as pointed out by the authors, not all studies omitted treatment with L-dopa; therefore, the observed effect on gastric emptying may sometimes be due to the medication used to treat the disease itself. Indeed, Robertson et al. reported a marked decrease in gastric emptying of a labelled paracetamol solution in healthy volunteers after oral administration of L-dopa. Mean time to $90 \%$ gastric emptying increased approximately 2.5 fold after prior oral intake of L-dopa (81 \pm 20 min vs. $32 \pm 24$ min, respectively) (Robertson et al., 1990). In an attempt to investigate the effect of Parkinson's disease on gastric motility, Unger et al. visualized and compared gastric motility in response to a solid (beef) and liquid meal (dextrose solution) in patients and healthy controls using MRI. Based on the velocity and amplitude of antral contraction waves, the authors calculated a motility index for each subject. Due to a significant decrease in amplitude compared to controls $(5.35 \pm 2.85 \mathrm{~mm}$ vs. $8.06 \pm 2.31 \mathrm{~mm}$, respectively), a trend towards a decrease in motility index was reported for Parkinson's disease patients (mean: $11 \pm 6.9$ vs. $16.5 \pm 5.1 \mathrm{~mm} \cdot \mathrm{s}^{-1}$, respectively (Unger et al., 2010). However, it should again be pointed out that in some cases a drug effect could not be ruled out, as only six out of ten patients did not take any medication to treat their condition.

Hermann et al. investigated the effect of diabetes mellitus on gastric emptying after intake of a mixed solid/liquid meal and on the bioavailability of thioctic acid enantiomers after oral dosing. Compared to controls, gastric emptying time was markedly delayed in $50 \%$ of diabetes mellitus patients. With regard to pharmacokinetics and bioavailability of thioctic acid, no significant changes were observed compared to healthy controls. Remarkably, differences in $\mathrm{C}_{\max }$ and Area Under the Curve (AUC) were more pronounced when comparing patients with and without normal gastric emptying due to an apparently 
higher bioavailability in diabetes mellitus patients with normal gastric emptying compared to healthy controls. This observation even resulted in a marked difference in oral bioavailability of one of the enantiomer forms within the diabetes mellitus patient population $(25.0 \pm 4.7 \%$ vs. $19.2 \pm 6.9 \%$, respectively) (Hermann et al., 1998).

\section{The stomach's contribution to drug disposition}

Before reaching the main site of absorption, i.e. the intestine, a dosage form resides in the stomach for a certain period of time. As already illustrated, the stomach is a complex, constantly changing environment with regard to anatomy, gastric contents and motor function. Depending on drug properties and formulation strategy used, these ever-changing factors may have a profound effect on the gastrointestinal behaviour of a drug and subsequently its systemic pharmacokinetics (Fig. 5). Furthermore, the influence of simultaneous administration of a meal on several gastric processes affecting drug disposition is to be considered, as many drugs are commonly taken with food.

\subsection{Gastric residence}

\subsubsection{Liquids}

Considering the absence of a disintegration and dissolution step and the faster transfer from stomach to duodenum, absorption from a liquid dosage form (e.g. solution, syrup) is usually faster than from a solid dosage form (e.g. tablet, capsule) (Jones, 1988). Braghetto et al. reported an average GE $t_{1 / 2}$ of $34.9 \pm$ $24.6 \mathrm{~min}$ for $200 \mathrm{~mL}$ of water in healthy volunteers, while solid dosage forms may reside in the stomach for several hours (Braghetto et al., 2009). Lloyd et al. compared digoxin pharmacokinetics after intake of a solution, a tablet, and a liquid-filled capsule dosage form. As expected, tablet administration resulted in an increase in $\mathrm{t}_{\max }$ compared to administration of a solution (mean: $1.2 \mathrm{~h}$ vs. $0.6 \mathrm{~h}$, respectively). Furthermore, both AUC and $\mathrm{C}_{\max }$ were markedly lower when the tablet was ingested compared to the solution. Swallowing a capsule containing a digoxin solution resulted in similar $\mathrm{t}_{\max }, \mathrm{C}_{\max }$ and AUC compared to drinking the drug solution as such. It seems that in this specific case, the fasted stomach was capable of rapidly disintegrating a liquid filled capsule, resulting in similar pharmacokinetics as for 
the drug solution (Lloyd et al., 1978). Similarly, Chen et al. performed a study comparing the pharmacokinetics of a diclofenac solution to an immediate-release tablet. Again, maximum plasma concentrations and time to reach these concentrations markedly differed between dosage forms, both under fasted (mean $\mathrm{t}_{\max }: 0.25$ vs. $0.5 \mathrm{~h}$, mean $\mathrm{C}_{\max }: 1620$ vs. $1160 \mathrm{ng} \cdot \mathrm{mL}^{-1}$, respectively) and fed state conditions ( $\mathrm{t}_{\max } ; 0.17$ vs. $1.25 \mathrm{~h}, \mathrm{C}_{\max }: 506$ vs. 835 ng. $\mathrm{mL}^{-1}$, respectively). However, total systemic exposure was similar (Chen et al., 2015). Gutierrez et al. obtained different results when investigating pharmacokinetics of citalopram administered as an oral solution $(60 \mathrm{mg} / 60 \mathrm{~mL})$ or as a tablet $(60 \mathrm{mg})$ in fasted volunteers. $\mathrm{C}_{\max }, \mathrm{t}_{\max }$ and AUC values were observed within the limits of bio-equivalence. The high mean $\mathrm{t}_{\max }$ (4.09 vs. $4.00 \mathrm{~h}$ for solution and tablet, respectively) suggests slow absorption of citalopram regardless of the dosage form. The citalopram solution contained sorbitol (70\%), propylene glycol and mint flavour which could have affected gastric emptying. Furthermore, the authors did not report the volume(s) of water used to ingest both formulations, although this could affect absorption as well (Gutierrez and Abramowitz, 2000). The use of a solution rather than a solid dosage form could be advantageous for dosage forms which rely on an acidic gastric environment to facilitate drug release (Pabla et al., 2008). Vita et al. and Brancato et al. studied the efficiency of two levothyroxine dosage forms in patients on proton-pump inhibitors (PPI). In the case of a tablet, a PPI-induced increase in gastric $\mathrm{pH}$ reduced the amount of levothyroxine dissolved and impaired intestinal drug absorption compared to 'normal' gastric $\mathrm{pH}$. When switched from a tablet to an oral solution (mixture of ethanol and glycerol) containing the same daily dose, patients' thyroid-stimulating-hormone levels dropped significantly, indicating that levothyroxine disposition is unaffected by an increase in gastric $\mathrm{pH}$ when administered as a solution (Brancato et al., 2014; Vita et al., 2014).

\subsubsection{Indigestible solids}

The stomach is capable of retaining dosage forms and thus determines when drugs are presented to the intestine to allow for absorption to occur. Therefore, the rate of gastric emptying influences the rate of drug absorption. For some compounds (BCS Class I), gastric emptying even represents the rate-limiting step for intestinal absorption. Due to the fact that large, indigestible solid objects only empty from the stomach with strong fasted state contractions (typically phase III contractions; cfr. 3.4.2. Gastric 
emptying), gastric residence of these solid dosage forms is prone to large variability. Furthermore, numerous other factors such as gender, posture, age, pathological conditions and pharmacological modulation may contribute to the large variability in gastric residence time (GRT) reported in literature (cfr. 3.4.3 Population-related differences) (Nimmo et al., 1973).

For instance, Bass et al. observed large variability in the gastric residence time of larger tablets (> 2 $\mathrm{mm}$ ) in a fasted stomach (range: 5 - $200 \mathrm{~min}$ ) (Bass, 1993). Using ferromagnetic labelling, transit of a dosage form along the GI tract can be monitored after ingestion, a technique called Magnetic Marker Monitoring (MMM). Weitschies et al. applied this technique to indigestible capsules in order to monitor gastric residence of these capsules, reporting large variability similar to findings by Bass et al. (mean: 37 min; range: 1 - 185 min) (Weitschies et al., 2010; Weitschies et al., 1999). Furthermore, electronic drug delivery systems (e.g. Intellicap ${ }^{\circledR}$, Wireless Motility Capsule ${ }^{\circledR}$, Smartpill $^{\circledR}$ ) have been used to monitor gastric residence time, and by extension whole gut transit time, of large solid objects. These types of systems are orally ingested and eventually excreted, meanwhile gathering data on intraluminal $\mathrm{pH}$, temperature and/or pressure which is either sent to a wireless receiver worn by the volunteer or recovered from the system after excretion (Farmer et al., 2013; Koziolek et al., 2015a; Saad and Hasler, 2011; Stein et al., 2013; van der Schaar et al., 2013; Yuce et al., 2009). Using such a system, Koziolek et al. reported similar variability in time to gastric emptying of an Intellicap ${ }^{\circledR}$ capsule $(27 \times 11 \mathrm{~mm}$; Medimetrics, The Netherlands) in healthy volunteers (range: 7 - 202 min) (Koziolek et al., 2014a). An indigestible Heidelberg Capsule (HC) can be used to investigate gastric residence of an indigestible solid dosage form. The $\mathrm{HC}$ measures intraluminal $\mathrm{pH}$, making it possible to deduce the exact time of gastric emptying from a steep rise in $\mathrm{pH}$ (Mojaverian, 1996). Mojaverian et al. administered an enteric-coated aspirin-containing dosage form together with a Heidelberg capsule (HC) to fasting volunteers. Mean GRT of the HC capsule amounted to $0.8 \pm 0.5$ hours. An excellent correlation between capsule GRT and the onset of aspirin absorption was observed (Mojaverian et al., 1987). The behaviour of large, indigestible objects in the fasted stomach can be considered a model for what might happen to extendedrelease and delayed-release dosage forms in the stomach. For these types of dosage forms, 
prolonged/delayed gastric residence may lead to altered drug release characteristics and possibly changes in systemic exposure (cfr. 4.2 Dosage form disintegration).

\subsubsection{Food}

As discussed earlier, ingestion of food will alter the emptying behaviour of the stomach. The extent of this effect partly depends on food characteristics such as meal structure, caloric density, etc. ( $c f r$. 3.4.2 Gastric emptying). Naturally, this will affect the residence time of a co-administered drug in the stomach. Compared to the fasted state, gastric residence of a dosage form is typically prolonged in postprandial conditions (Koziolek et al., 2015a; Koziolek et al., 2015c; Mojaverian et al., 1987). For instance, Weitschies et al. reported a marked increase in gastric residence time of an indigestible capsule under fed state conditions compared to fasted state conditions (range: $69-583 \min$ vs. $1-185 \mathrm{~min}$, respectively) (Weitschies et al., 2010). Furthermore, by maintaining fed state conditions via the administration of a meal every 4 hours, Newton et al. were able to extend the gastric residence time of a pellet formulation (1.0 - $1.4 \mathrm{~mm}$ ) by several hours (Newton, 2010).

Additionally, residence of a dosage form in the fed stomach has been observed to be dosage form dependent. Depending on the size of the dosage form, for instance, gastric residence time can be markedly increased in the fed stomach (up to 15 hours) (Cortot and Colombel, 1984; Garbacz et al., 2008; Varum et al., 2013).Abrahamsson et al. compared the mean gastric emptying time (GET) of a multiple-unit formulation (pellets with diameter: $0.4-0.6 \mathrm{~mm}$ ) and a non-disintegrating tablet (diameter: $9 \mathrm{~mm})$. The tablet emptied significantly slower from the stomach compared to the pellet formulation (mean GET: 9.6 vs. 3.3 h, respectively) (Abrahamsson et al., 1996).

\subsection{Gastric emptying}

Intestinal drug absorption is initiated as soon as a drug reaches the site of absorption. Therefore, drug transfer from the stomach to the small intestine influences subsequent intestinal absorption. Moreover, in the case of compounds without solubility and permeability restrictions (i.e. BCS class I) gastric emptying is the rate-limiting step determining drug absorption (immediate-release dosage forms) (Prescott, 1974). For example, a good correlation between $\mathrm{GE}_{1 / 2}$ and systemic $\mathrm{C}_{\max }$ has previously been 
reported for paracetamol (Heading et al., 1973). Using a mathematical compartmental model, Talattof et al. studied the effect of fasted state gastrointestinal motility variation on plasma levels for highsolubility compounds (BCS classes I and III). The study indicated that gastric motility may indeed have an important impact on systemic drug pharmacokinetics and that in some cases bioequivalence studies may fail solely due to natural variations in gastrointestinal motility (Talattof et al., 2016).

Studies investigating the effect of gastric emptying on drug absorption often use motility-stimulating (e.g. metoclopramide, erythromycine) or motility-inhibiting (e.g. propantheline, loperamide) agents (Marathe et al., 2001; Nimmo et al., 1973). For example, Marathe et al. used such agents to investigate the influence of gastric emptying on metformin absorption from an oral solution in healthy volunteers. Metformin absorption correlated well with gastric emptying of the drug solution. While propantheline pretreatment resulted in an increase in $t_{\max }$, metoclopramide pretreatment decreased $t_{\max }$ (mean: 3.5 versus $2.5 \mathrm{~h}$, respectively). Overall exposure remained unchanged after metoclopramide pretreatment but increased after propantheline pretreatment compared to controls (mean increase: $+19 \%$ ). The authors attributed the latter finding to an overall longer GI transit time for metformin, a BCS class III compound, improving its extent of intestinal absorption (Marathe et al., 2001). Similar changes in $t_{\max }$ were reported for cefprozil (BCS class II) capsules after motility-changing pretreatments. However, for this compound overall systemic exposure did not differ between the conditions tested (Shukla et al., 1992). Lastly, Knupp et al. observed an increase in mean maximal systemic didanosine concentrations in asymptomatic HIV patients pretreated with metoclopramide versus those receiving loperamide pretreatment ( $2.30 \pm 0.80$ vs. $1.50 \pm 0.86 \mu \mathrm{g} . \mathrm{mL}^{-1}$, respectively) (Knupp et al., 1993a).

Multiple techniques have been applied to investigate gastric emptying behaviour of both solid and liquid components (e.g. gamma-scintigraphy, ${ }^{13} \mathrm{C}$-octanoic acid breath test, ultrasonography, electrogastrography, MRI) (Banerjee et al., 2014; Bolondi et al., 1985; Goetze et al., 2006; Madsen, 2013; Marzio et al., 1989; Perri et al., 2005). For example, gamma-scintigraphy, in which a meal/dosage form is radio-isotopically labelled and content movement in the GI tract is visualized using a gammacamera, is often used to investigate gastric emptying of solid and liquid meals and/or dosage forms (Davis et al., 1992; Madsen, 2013). Using this technique, Coupe et al. reported large inter-individual 
variability in gastric emptying of a non-disintegrating dosage form in lightly fed volunteers (Coupe et al., 1991). The ${ }^{13} \mathrm{C}$-octanoic acid breath test provides a valuable non-radioactive alternative to gammascintigraphic measurements. This test relies on the intestinal absorption, rate-limited by gastric emptying, and metabolism of ${ }^{13} \mathrm{C}$-octanoic acid, which is subsequently exhaled as ${ }^{13} \mathrm{CO}_{2}$. As results obtained from this test correlated well with those obtained using gamma-scintigraphy, the ${ }^{13} \mathrm{C}$-octanoic acid breath test is considered a safer, less-invasive method to measure gastric emptying of solids (Ghoos et al., 1993; Perri et al., 2005). Maes et al. used the breath test to detect pharmacologically induced changes in gastric emptying of a solid meal. Healthy volunteers were administered either erythromycin or propantheline to increase or decrease gastric emptying of a solid meal compared to controls, respectively. As hypothesized by the authors, administration of erythromycin indeed resulted in a decrease in GE $t_{1 / 2}$, while the opposite was observed after propantheline administration (Maes et al., 1994).

Hens et al. used paromomycin (BCS class III) as a non-absorbable GI marker to investigate gastric emptying of drug solutions. By determining intraluminal drug concentrations in healthy volunteers after administration of a paromomycin solution, gastrointestinal transfer rates could be studied. Compared to fasted state conditions, intake of a liquid meal slowed gastric emptying of the solution (mean GE $\mathrm{t}_{1 / 2}$ : 5.3 vs. $33 \mathrm{~min}$, respectively). However, even in the fed state, part of the solution rapidly transferred to the duodenum. The authors suggested that this finding may be related to the 'magenstrasse' phenomenon (Hens et al., 2014). The magenstrasse scenario is an interesting hypothesis with regard to gastric emptying of dosage forms from the stomach after ingestion of a liquid meal. In a publication by Pal et al., the traditional assumption that gastric content is only directly emptied from the distal stomach is challenged. After creating a 2D, computational fed stomach model based on in vivo stomach geometry data, the authors investigated particle flow patterns and gastric emptying after simulating the ingestion of a liquid meal. Their findings suggest the existence of a narrow path along the lesser curvature of the stomach stretching from the fundus all the way to the pylorus, facilitating the rapid emptying of gastric contents from the upper stomach and bypassing adequate mixing of food with gastric secretions. By varying geometrical parameters (i.e. occlusion, contraction width), the formation and shape of this 
magenstrasse was observed to change, suggesting a clear link between the magenstrasse phenomenon and fed state gastric motility (Pal et al., 2007). Rapid emptying of contents on the magenstrasse may lead to rapid appearance of drug in the duodenum and subsequently a rapid onset of intestinal absorption. For example, similar to earlier observations, Van Den Abeele et al. reported maximal duodenal drug concentrations within 12 minutes after oral administration of a diclofenac potassium solution for four out of five fed subjects (Van Den Abeele et al., 2015).

\subsection{Dosage form disintegration}

A solid dosage form needs to disintegrate in order for the drug to be released and subsequently absorbed. As the transit time of a dosage form through the mouth and esophagus is short, the stomach is often the first part of the GI tract where dosage form disintegration may occur (Cordova-Fraga, 2008). Gastric motor function facilitates dosage form disintegration via surface erosion (mixing) and by peristaltic contractile forces acting directly on the dosage form. Jain et al. visualized the erosion of HPMC matrix tablets in man using Magnetic Marker Monitoring under both fasted and fed state conditions. Tablet erosion was observed at the level of the stomach, with a greater contribution of the stomach in fed state (longer residence time + prolonged contact with gastric content) (Jain et al., 2014). With regard to dosage form fractionation, Kamba et al. attempted to quantify the forces exerted by the gastric wall on a solid dosage form by orally administering tablets with very specific fractionation strengths (1.50 and $1.89 \mathrm{~N}$, respectively; tablet diameter: $7 \mathrm{~mm}$ ) to both fasted and fed individuals. Results indicated that under fed state conditions, higher crushing strengths (up to $1.89 \mathrm{~N}$ ) act on the administered tablets compared to fasted state conditions $(1.50 \mathrm{~N})$. The authors hypothesized that grinding of the tablet against a closed pylorus, which is more probable in fed individuals, results in high crushing strengths and hence would explain their observation (Kamba et al., 2000). In a similar fashion, Marciani et al. reported antral contraction strengths of $0.53-0.78 \mathrm{~N}$ in healthy subjects after a meal. An increase in meal viscosity accelerated gastric emptying of beads, suggesting that transfer of gastric contraction forces onto the beads is more efficient with increasing meal viscosity (Marciani et al., 2001a). However, as gastric 
motor function (i.e. contraction frequency and amplitude) is both site- and time-dependent (cfr. 3.4.1 Gastric motility), dosage form disintegration in the stomach can be prone to marked variability. For instance, Weitschies et al. observed substantial variation in systemic pharmacokinetics of extendedrelease (ER) felodipine tablets. These pharmacokinetic differences could be traced back to the increased proximal retention of the dosage form, resulting in marked changes in drug release (Weitschies et al., 2005b). Furthermore, close contact between the dosage form and the gastric wall may in some cases have a pronounced effect on drug release due to adherence of the dosage form to the stomach lining. For instance, Hey et al. observed that a pivampicillin capsule formulation was prone to become stuck in the membranous folds of the gastric wall, a phenomenon which was not observed for pivampicillin tablets (Hey et al., 1979). While the tablets disintegrated fast, the capsules settled in the mucous layer lining the gastric wall. Adherence of a formulation to the gastric wall may result in very local drug release, potentially causing mucosal damage at the release site (Graham et al., 1990; Hey et al., 1979). In the study by Hey et al., fifty percent of volunteers suffered from side effects caused by local injury to the gastric membrane after intake of the capsule formulation, whereas none of the volunteers experienced side effects after intake of the tablet formulation (Hey et al., 1979).

Radwan et al. investigated the possible effect of the presence of food in the stomach on tablet disintegration. Changes in medium viscosity, water diffusivity and hydrodynamics as well as precipitation of food constituents on the tablet surface were all found to potentially drastically reduce the rate of dosage form disintegration (Radwan et al., 2012; Radwan et al., 2013; Radwan et al., 2014). By sampling intraluminal contents via aspiration catheters positioned in the stomach, the gastric behaviour of orally administered drugs can be elucidated (Brouwers et al., 2007; Hens et al., 2016a; Van Den Abeele et al., 2015; Vidon et al., 1989; Walravens et al., 2011). Using this technique, Brouwers et al. investigated the GI behaviour of fosamprenavir both under fasted and fed state conditions. The authors observed a food-induced delay in in vivo tablet disintegration after administering a fosamprenavir tablet together with a liquid meal (Scandishake Mix $\left.{ }^{\circledR}\right)$, resulting in delayed intestinal absorption for this compound (Brouwers et al., 2007). In vitro MRI studies revealed that tablet 
disintegration was delayed due to impaired ingression of water when the tablet was coadministered with the liquid meal (Brouwers et al., 2011).

In the case of immediate-release dosage forms, rapid intragastric disintegration is required in order not to delay drug absorption. By altering dosage form properties, the process of disintegration can be markedly influenced (Katori et al., 1996; Shameem et al., 1995; Shirsand et al., 2010). RostamiHodjegan et al. investigated the effect of a fast-disintegrating tablet formulation containing sodium bicarbonate on systemic paracetamol exposure compared to a conventional tablet formulation in healthy volunteers. Although overall systemic exposure was similar, $\mathrm{t}_{\max }$ was significantly shorter for the fastdisintegrating formulation compared to the conventional formulation, under both fasted $(25 \pm 18 \mathrm{~min}$ vs. $55 \pm 28 \mathrm{~min}$, respectively) and fed state conditions ( $45 \pm 35 \mathrm{~min}$ vs. $120 \pm 47 \mathrm{~min}$, respectively) (Rostami-Hodjegan et al., 2002).

Co-ingestion of an extended- or delayed-release dosage form with food extends its gastric residence ( $c f r$. 3.4.2. Gastric emptying) and thus prolongs the dosage form's exposure to mixing and fractionation forces in the stomach, increasing the risk for erratic drug release kinetics and so-called 'dose dumping' scenarios (Hendeles et al., 1985; Karim et al., 1985; Marvola et al., 1989; Steffensen and Pedersen, 1986). In 1985, Hendeles et al. reported food-induced dose dumping for a once-a-day theophylline dosage form (Theo-24). An average 2.3-fold increase in systemic $\mathrm{C}_{\max }$ was observed in healthy volunteers when oral intake of the dosage form was accompanied by a breakfast, resulting in toxic effects in 50\% of all cases (Hendeles et al., 1985). Similar observations of dose dumping have been reported for other first generation theophylline controlled release formulations (Karim et al., 1985; Steffensen and Pedersen, 1986). Schug et al. performed multiple bioequivalence studies, comparing several HPMCbased ER dosage forms of nifedipine to a well-established osmotic-controlled release oral delivery system $\left(\mathrm{OROS}^{\circledast}\right.$, Adalat $\left.\mathrm{OROS}^{\circledR}\right)$. Similar as for other $\mathrm{OROS}^{\circledR}$ dosage forms, the reference product displayed robust behaviour regardless of prandial state (Cortot and Colombel, 1984; Sathyan et al., 2007; Schug et al., 2002a; Schug et al., 2002b; Schug et al., 2002c; van den Berg et al., 1990). In contrast, the HPMC-based dosage forms displayed drastic changes in systemic pharmacokinetics after intake with food. For two dosage forms, $\mathrm{C}_{\max }$ increased 2.3- and 3.4-fold compared to the Adalat OROS $^{\circledR}$ dosage 
form, while for a third dosage form the onset of absorption was markedly delayed (up to 15 hours in $50 \%$ of cases) resulting in a drastically lower bioavailability after 24 hours (Schug et al., 2002a; Schug et al., 2002b; Schug et al., 2002c). As drug release from hydrophilic matrix tablets is erosion-controlled, dose dumping for these formulations has been ascribed to a faster and more extensive surface layer erosion in the fed stomach compared to the fasted state, resulting in marked (toxic) changes in drug exposure (Abrahamsson et al., 1998; Abrahamsson et al., 1999; Garbacz et al., 2009; Jain et al., 2014). In line with these findings, Jain et al. observed that by increasing HPMC concentrations and molecular weight, the in vivo erosion rate can be slowed down (Jain et al., 2014). However, Magnetic Marker Monitoring-findings by Weitschies et al. suggest that higher systemic exposure under fed state conditions compared to fasted state conditions for ER tablets may not always be ascribed to erratic dosage form performance, but also to the intragastric location of the tablets. As a decline in magnetic signal due to dosage form disintegration could be linked to drug release, the relative impact of the dosage form's intragastric location on said drug release could be investigated (Weitschies et al., 2001; Weitschies et al., 2005a). In this study, tablets residing in the proximal stomach for a longer period of time were more likely to result in higher systemic peak concentrations after intestinal absorption. It is however not obvious that this is due to more extensive tablet erosion in the proximal stomach, as in this region of the stomach mixing of gastric contents is typically less pronounced. More likely, the combination of an increase in gastric residence time, local drug release and poor mixing in the proximal stomach results in high regional drug concentrations which, when emptied from the stomach to the duodenum, may lead to high systemic peak concentrations (Weitschies et al., 2005b).

\subsection{Drug dissolution, supersaturation and precipitation}

In parallel with dosage form disintegration, drug dissolution takes place. Using specific formulation strategies or exploiting specific traits of the GI tract, drug can be present in a supersaturated state. Supersaturation is a metastable state in which drug concentrations in solution exceed their thermodynamic solubility. Due to the thermodynamically metastable nature of the supersaturated state, drug precipitation is inevitable. By achieving supersaturated drug concentrations at the site of absorption (i.e. the intestine), bioavailability of poorly-soluble drugs can be improved (Brouwers et al., 2009). In 
theory, gastric supersaturation of weakly acidic BCS class II drugs may occur in vivo due to the limited solubility of these compounds in acidic media. Van Den Abeele et al. investigated the possibility of gastric supersaturation in healthy volunteers following oral administration of a diclofenac potassium solution. Modest supersaturated concentrations of diclofenac could be detected in only four gastric aspirates, indicating that gastric supersaturation may only be briefly present for this compound. These findings corroborated earlier in vitro findings by Bevernage et al., who stated that gastric fluids may not be a favourable environment for the stabilization of supersaturation (Bevernage et al., 2012). Furthermore, all volunteers displayed extensive precipitation of diclofenac in the stomach, the extent of which was found to be well-correlated with gastric $\mathrm{pH}$. Although precipitation may potentially influence intestinal absorption kinetics and thus systemic pharmacokinetics, this was not the case in the diclofenac study due to rapid intestinal re-dissolution of solid material transferred from the stomach into the duodenum (Van Den Abeele et al., 2015).

Furthermore, the stomach may be very important for the creation of drug supersaturation at the level of the small intestine for compounds which display medium-dependent solubility. In case of higher solubility in the gastric environment compared to the small intestine, drug transfer from the stomach to the duodenum may induce intestinal supersaturation. This has been observed to occur in vivo for several poorly water soluble, weakly basic drugs (Hens et al., 2016a; Psachoulias et al., 2011). Hens et al. studied the ability of posaconazole to supersaturate in the duodenum and its implications for systemic exposure following intragastric administration of both a neutral and an acidified drug suspension ( $\mathrm{pH}$ 7.1 and 1.6, respectively). Administration of the acidified suspension resulted in duodenal supersaturation for approximately 45 minutes. In contrast, supersaturation was absent following administration of the neutral suspension. As a result, a two-fold increase in systemic exposure was observed when comparing the acidified to the neutral suspension. These observations illustrate the importance of a sufficiently acidic stomach in generating intestinal supersaturation of weakly basic drugs, which may in turn result in more efficient drug absorption (Hens et al., 2016a). Changes in gastric pH due to population-related differences, pharmacological agents (e.g. PPIs) or pathological conditions (cfr. 3.3. Gastric fluid composition) may affect drug solubility and thus the potential to induce 
supersaturation upon transfer to the duodenum. In addition, food ingestion will induce a variety of changes in the composition of intraluminal fluids which in turn will influence supersaturation and precipitation behaviour of ionizable compounds (cfr. 3.3. Gastric fluid composition). Van Den Abeele et al. observed a marked increase in gastric $\mathrm{pH}$ after liquid meal consumption. Initial $\mathrm{pH}$ values closely resembled that of the ingested meal ( $\mathrm{pH}$ 6.55), after which $\mathrm{pH}$ gradually decreased back to basal values over the course of the following hours. This temporary increase in $\mathrm{pH}$ favours the dissolution and solubility of weakly acidic drugs in the stomach. As a result, Van Den Abeele et al. did not observe intragastric supersaturation and precipitation of the BCS Class II weak acid diclofenac, contrary to observations in the fasted state (Van Den Abeele et al., 2015). Likewise, this temporary change in gastric $\mathrm{pH}$ (and thus solubility) could affect supersaturation of weakly basic drugs upon transfer to the duodenum. As the $\mathrm{pH}$ difference between stomach and intestine is less pronounced in a fed state, the solubility gradient between both GI compartments and thus the ability to generate supersaturation is largely lost.

\subsection{Absorption from the stomach}

The main site of drug absorption in the GI tract is the duodenum. Due to its high surface area and the presence of a large variety of transporters, the duodenum represents a more suitable site of absorption than the stomach (Holt, 1981). Drug absorption from the stomach is generally thought to be negligible relative to overall absorption. However, passive diffusion of small neutral molecules (e.g. alcohol, small molecular drugs) through the gastric mucosa seems plausible. For instance, Levitt et al. estimated that absorption from the stomach accounts for $10 \%$ of the total ethanol absorption under fasted state conditions. Under fed state conditions, up to $30 \%$ of ingested ethanol is absorbed from the stomach (Levitt et al., 1997). Challenging the general consensus, several authors have suggested that the gastric mucosa can be considered a mere lipophilic barrier facilitating passive diffusion of unionized drug (Hogben et al., 1957; Jaques et al., 1991; Schanker et al., 1957). Unfortunately, studies on gastric drug absorption in humans are scarce and often dated. In 1957, Schanker et al. and Hogben et al. performed the first studies regarding gastric absorption in both rats and humans (Hogben et al., 1957; Schanker et al., 1957). The authors estimated the amount of drug absorbed through the gastric wall by directly 
sampling gastric content, correcting for dilution effects using phenol red as a non-absorbable marker. The difference between the initial drug amount and the drug amount recovered was presumed to be absorbed from the stomach (Hogben et al., 1957; Jobin et al., 1985; Schanker et al., 1957; Vidon et al., 1989). Hogben et al. measured gastric absorption of eight drugs, administered as a solution in either water or $0.1 \mathrm{M} \mathrm{HCl}$, in three healthy volunteers. Administered as a $0.1 \mathrm{M} \mathrm{HCl}$ solution, five out of eight drugs were found to be absorbed through the gastric wall. Not surprisingly, all weakly acidic drugs (pKa $>3$ ) were absorbed as these are present in the acidic solution in their unionized form. The only basic compound absorbed from the stomach was the very weak base antipyrine (pKa 1.4), whereas other basic compounds (quinine, ephedrine and aminopyrine) were not absorbed. The percentage of gastric drug absorption after 20 minutes ranged from $58.0 \pm 13.4 \%$ (acetyl salicylic acid) to $8.3 \pm 4.0 \%$ (antipyrine). Administered with water, all drugs were found to be absorbed to some extent through the gastric wall. Acidic drugs were readily absorbed, whereas basic drugs were only absorbed to a very small extent. The percentage of drug absorption after 20 minutes ranged from $62.0 \pm 11.4 \%$ (acetyl salicylic acid) to 1.7 $\pm 2.9 \%$ (quinine). The authors attributed this finding to the rapid re-acidification of gastric contents after ingestion of the solution. Hogben et al. concluded that mainly acidic drugs in their unionized form could be absorbed through the gastric mucosa. The results of this study were compared with gastric absorption studies using the same compound set in rats, revealing a good correlation between the gastric absorption of drugs in humans and rats (Hogben et al., 1957; Schanker et al., 1957). Other researchers continued to use the same method with alternative non-absorbable markers (e.g. PEG-4000, [14C]PEG) for the further study of gastric drug absorption (Jobin et al., 1985; Vidon et al., 1989). Jobin et al. investigated the absorption of metoprolol (weak base) along the GI tract. Metoprolol was found not to be absorbed from the stomach (Jobin et al., 1985). Vidon et al. investigated gastric absorption of diclofenac (weak acid) in healthy volunteers. Gastric absorption was not observed when co-administered with $400 \mathrm{~mL}$ of water or phosphate buffer $\mathrm{pH}$ 7.5. Only some gastric absorption was detected when co-administered with a homogenized meal. Yet, this did not affect plasma drug concentration-time profiles (Vidon et al., 1989). It is clear that conflicting reports on drug absorption from the stomach warrant intensified efforts to further investigate this controversial topic. 


\subsection{Dosage form processing/digestion}

Dosage form residence in the stomach provides not only the opportunity for mechanical degradation (cfr. 4.2. Dosage form disintegration) but also for chemical and enzymatic 'digestion' of the dosage form (cfr. 3.3. Gastric fluid composition). These features may either be beneficial/essential or detrimental to drug disposition. For instance, gastric residence prior to transfer to the duodenum plays an important role in the bioavailability of drugs formulated using a lipid-based formulation (LBF) strategy. Dosage form dispersion and digestion are essential steps to enable drug absorption from these types of formulations. Lee et al. compared the bioavailability of a cinnarizine-containing LBF after intraduodenal and per oral administration in rats. The stomach was found to pre-disperse the dosage form, hereby delivering a partially digested, supersaturated colloidal dispersion to the duodenum. Bypassing the gastric compartment resulted in a decrease in cinnarizine bioavailability (Lee et al., 2013). Due to the high amount of lipids present in the formulation, LBF digestion also indirectly increases absorption by slowing gastric emptying (Kossena et al., 2007; Matzinger, 2000). Especially long-chain triglycerides delay gastric emptying and intestinal transit and thus increase residence time of food and drugs in the small intestine, potentially leading to improved absorption (Matzinger, 2000). Using videoendoscopic technology (Pillcam ${ }^{\circledR}$, Given Imaging, Israel), Pedersen et al. visualized the behaviour of LBFs in the human stomach (Eisen et al., 2006; Schoofs et al., 2006). By fixing different LBFs to a miniaturized endoscope, the authors were able to visualize dosage form disintegration, dispersion characteristics as well as the impact of the gastric environment on a specific dosage form (Pedersen et al., 2014).

\subsection{Drug degradation}

Passage of a dosage form through the stomach can lead to undesirable effects, due to the specific features of the gastric environment. For instance, compounds can be sensitive to acid hydrolysis and/or minor gastric metabolism. For this reason, therapeutic proteins (insulin, antibodies) are almost exclusively administered intravenously. Although the advantages of an oral dosage form over intravenous administration stimulate efforts to enable oral delivery of therapeutic proteins, their development 
remains challenging. Apart from their low permeability, oral administration of these proteins leads to intraluminal degradation due to gastric (and intestinal) peptidase activity and subsequently to poor bioavailability (Crane and Luntz, 1968; Fonte et al., 2013; Singh et al., 2008; Ziv and Bendayan, 2000). Current research therefore focuses on using polymers (mechanisms similar to extended release formulations) and liposome encapsulation techniques as ways to circumvent the harmful gastric environment (Fonte et al., 2013; Singh et al., 2008). Furthermore, several broadly used small molecules are acid-labile. For example, macrolide antibiotics rapidly degrade under acidic conditions (Fiese and Steffen, 1990; Kristl and Vrečer, 2000; Nakagawa et al., 1992). Similarly, PPIs and certain penicillin antibiotics are acid-labile (Blaha et al., 1976; Finholt et al., 1965; Lim et al., 2011; Pilbrant and Cederberg, 1985). Knupp et al. used pentagastrin-pretreated dogs to evaluate the acid-stability of didanosine. Didanosine bioavailability decreased more than five-fold in pentagastrin-pretreated dogs compared to untreated animals (Knupp et al., 1993b). Likewise, Kosugi et al. evaluated the acid tolerance of drugs using $\mathrm{pH}$-controlled rat and dog models. Gastric $\mathrm{pH}$ was controlled via stimulation or inhibition of gastric acid secretion. The ratio between drug exposure with or without stimulation of gastric acid secretion $\left(\mathrm{AUC}_{\text {acid inhibited }} / \mathrm{AUC}_{\text {acid stimulated }}\right)$ was suggested as an indicator whether or not a drug is stable in an acidic environment. Omeprazole and erythromycin were found to be acid-labile (ratio > 1.9), whereas clarithromycin, azithromycine and etoposide (ratio < 1.3) were more acid stable (Kosugi et al., 2015).

\section{Formulation strategies with gastric involvement}

As illustrated earlier, drug disposition can both benefit from (e.g. slowly dissolving drugs, weakly basic drugs) and be harmed by (e.g. acid-labile compounds, extended/delayed release dosage forms) passage through the stomach (cfr. 4. The stomach's contribution to drug disposition). In order to either exploit or avoid the influence of the gastric environment on drug disposition, several formulation strategies have been developed to ensure or prevent intragastric drug release.

\subsection{Targeting the stomach}


Gastroretentive drug delivery systems (GDDS) aim to prolong a dosage form's residence in the stomach. Especially weakly basic drugs with poor solubility in neutral environments and drugs with narrow absorption windows in the upper part of the GI tract may benefit from these approaches. Prolonged residence may ensure complete dissolution in the stomach and/or sustained exposure to the duodenum for these compounds and may thus benefit systemic exposure. Mechanisms such as mucoadhesion, floatation, sedimentation, expansion or modified shape systems have been pursued in order to obtain sufficient gastroretention of dosage forms. For example, with regard to the latter strategy tetrahedronand ring-shaped dosage forms generally reside in the stomach for a longer period of time compared to other shapes (Arora et al., 2005). Similarly, Kedzierewicz et al. reported that under fed state conditions, slab and rod shapes reside longer in the stomach compared to minimatrices (Kedzierewicz et al., 1999). For an in-depth-description of the mechanisms and formulation technology, the reader is referred to reviews by Streubel et al. and Arora et al. (Arora et al., 2005; Streubel et al., 2006).

Many drugs have been formulated as floating delivery systems and have been tested in vivo in both animals and humans. A comprehensive overview of these studies is provided by Arora et al. (Arora et al., 2005). Currently marketed floating delivery systems, containing a large variety of compounds such as antacids, misoprostol, ciprofloxacin, diazepam, levodopa and benserazide, use a multitude of mechanisms in order to achieve dosage form floatation (Dwivedi and Kumar, 2011). Although many reports claim a beneficial effect of floating delivery systems on systemic drug exposure, not all compounds may benefit from this formulation approach. For example, Menon et al. reported a marked increase in absolute furosemide bioavailability in beagle dogs when formulated as a floating delivery system compared to the commercially available immediate-release tablet (mean: 42.9 vs. $33.4 \%$, respectively) (Menon et al., 1994). However, Timmermans et al. demonstrated that external factors such as body position (and thus stomach shape and position) may have an important influence on the success and therapeutic reproducibility of this specific formulation strategy. Using the floatation approach, the authors compared gastric residence time of a floating versus a non-floating delivery system in fed subjects both in upright and supine position. While in upright position gastric residence was significantly prolonged in the case of the floating system, this difference was not observable with subjects in supine 
position (Timmermans and Moes, 1994). The difference in performance of the floating system observed by Timmermans et al. is most likely due to a difference in dosage form sensitivity to peristaltic contraction waves depending on the subjects' posture. Moreover, other authors have challenged the added benefit of using buoyant dosage forms to improve drug disposition (Davis et al., 1986; Rouge et al., 1998). For instance, Rouge et al. even observed a minor decrease in atenolol bioavailability when formulated as a floating dosage form. The use of a high-density formulation in order to obtain gastric retention and increase systemic exposure yielded similar unsatisfactory results in the same study (Rouge et al., 1998).

\subsection{Protective formulation strategies}

An often-used strategy to protect the active compound from the harsh gastric environment is the application of a polymer coating. The appropriate coating is chosen based on (i) its reluctance to dissolve in acidic media and (ii) its ability to rapidly dissolve once in the duodenum (Bash and Troy, 2015). Frequently used coating polymers are shellac, cellulose acetate phthalate (CAP), polyvinyl acetate phthalate (PVAP), cellulose derivatives (e.g. HPMCP, HPMCAS) and methacrylic acid copolymers (Eudragits) (Irving R, 1991). pH-sensitive polymers are currently used in formulation strategies for acidlabile drugs, drugs known to irritate the gastric wall (e.g. NSAIDs), drugs with variable absorption depending on gastric conditions and in the case of targeted duodenal/colonic drug release (Felton, 2011; Graham et al., 2005; Hens et al., 2016b; Lanza, 1984). Ideally, a coating should be resistant to the wide range of reported gastric $\mathrm{pH}$ values for its entire gastric residence (cfr. 3.3.2. Acidity of gastric content). Due to a rise in gastric $\mathrm{pH}$, administering enteric-coated dosage forms to fed subjects may prevent delayed drug release, rendering the formulation strategy futile. Kenyon et al. investigated the disintegration behaviour of enteric-coated capsules in healthy volunteers both under fasted and fed state conditions. Although capsule integrity was unaffected in both conditions, capsules residing longer in the fed stomach disintegrated faster once emptied in the duodenum. The authors attributed this finding to the fact that the enteric coating, although insoluble in gastric fluids, could still be permeated by gastric fluids. The permeated gastric fluids could then dissolve the underlying contents, which could subsequently be released faster in the duodenum. However, it is plausible that the temporary elevation 
in gastric $\mathrm{pH}$ could have already weakened the enteric barrier and thus primed it for faster duodenal drug release (Kenyon et al., 1994). Similarly, Digenis et al. reported more rapid disintegration of entericcoated erythromycin pellets in fed compared to fasted subjects. Softening of the enteric coating due to elevated intragastric $\mathrm{pH}$ and milling of the food-drug content in the stomach would explain this observation (Digenis et al., 1990). Vaz-da-Silva et al. tested two enteric-coated omeprazole dosage forms in both fasted and fed state. Following a high-caloric, high-fat meal, the rate and extent of systemic exposure for both products was significantly reduced. Although the authors did not mention a pH effect, it may be possible that a temporary elevation of the gastric $\mathrm{pH}$ caused premature drug release, resulting in degradation of the acid-labile compounds as the stomach re-acidifies and subsequently reducing the amount of drug absorbed (Vaz-da-Silva et al., 2005). With respect to colonic drug release, polymers which are soluble only above $\mathrm{pH} 7$ or which can only be digested by colonic microbiota (e.g. containing azo-bonds, polysaccharides) are often used (Chourasia and Jain, 2004; Philip and Philip, 2010; Rajpurohit et al., 2010).

Furthermore, other mechanisms have been explored as well in order to ensure that initiation of drug release is postponed to the level of the duodenum. For instance, Wilde et al. developed a dosage form capable of pulsatile drug delivery when exposed to pressures greater than 300 mbar. The dosage form consists of a firm outer shell and a core which liquidizes at body temperature. As the dosage form empties from the stomach, the outer shell breaks upon passage through the pylorus, subsequently facilitating drug release in the duodenum (Wilde et al., 2014). Although this type of formulation strategy has proven its merits in an in vitro setting, it is nevertheless risky to rely on intragastric pressure events to initiate in vivo drug release. Inter- and intra-individual variation in gastric contraction strength may lead to either early or impaired breaking of the pressure-sensitive dosage form (cfr. 3.4.1. Gastric motility). Niwa et al. developed a capsule for colonic drug delivery which only releases drug several hours after oral ingestion. The dosage form relies on water ingression through micropores in the capsule, causing polymer swelling to the point that the capsule can no longer resist the increasing internal pressure and bursts. The capsule is meant to pass through the stomach and small intestine without releasing drug (Niwa and Jones, 1996). This mechanism is somewhat similar to an OROS $^{\circledR}$ tablet, for 
which swelling does not cause the capsule to rupture but rather pushes the drug through a small laserdrilled hole in the shell (Conley et al., 2006). Again, relying on gastric physiology to ensure site-specific drug release poses a considerable risk of erroneous drug release due to potentially large intra- and interindividual physiological differences (cfr. 4.1. Gastric residence).

In this context, ingestible electronic drug delivery systems may be a valuable alternative ensuring drug release at the intended target site. These systems contain both a reservoir in which a certain dosage form (e.g. solution, powders, particles) can be stored and a delivery pump, expelling the reservoir's content to a specific region of interest based on real-time measurements of intraluminal $\mathrm{pH}$ (van der Schaar et al., 2013). For example, Beckers et al reported that by programming a diltiazem-containing electronic drug delivery system $\left(\right.$ Intellicap ${ }^{\circledR}$ ) to initiate drug release at the level of the colon, bioequivalent systemic exposure could be obtained compared to the traditional colon-targeting slow-release diltiazem formulation (Becker et al., 2014).

\section{Influence of real-life dosing conditions on drug disposition}

Clinical trials are conducted under strictly controlled circumstances, including strictly defined protocols for dosage form administration to the study subject. In the case of bioavailability/bioequivalence studies for oral dosage forms, the dosage form is required to be administered with a certain amount of water ranging from 150 to $240 \mathrm{~mL}$ of water depending on the regulatory authority involved (CHMP, 2010; FDA, 2014b). However, once marketed, administration conditions are far less controlled. For instance, very few patients pay attention to the volume of water that is used to take an oral dosage form, although changes in intragastric volume and $\mathrm{pH}$ may have an important influence on the behaviour of active compounds and may thus influence drug absorption kinetics and bioavailability. Moreover, some patients may even take a dosage form with other beverages than the intended water, e.g. sodas, milk, fruit juices, hot beverages and/or alcoholic beverages. These altered or 'real-life' dosing conditions may influence drug absorption as factors such as intragastric temperature, $\mathrm{pH}$ and volumes may be altered as well (Fig. 5). 


\subsection{Concomitant use of anti-secretory therapy}

Polypharmacy is a global phenomenon, typically increasing in incidence with age (Bjerrum et al., 1998; Tseng et al., 2013). Therefore, drug-drug interactions may occur quite regularly in daily practice. One of the most prescribed drugs in this context are the acid secretion-inhibiting drugs, more specifically PPIs, which are prescribed for a multitude of indications (Haastrup et al., 2014; Heidelbaugh et al., 2012; Rakesh, 2011). These types of drugs inhibit gastric acid secretion via several different pathways. For instance, PPIs inhibit gastric acid secretion by irreversibly binding to $\mathrm{H}^{+} / \mathrm{K}^{+}$ATPase in parietal cells, while $\mathrm{H}_{2}$-antagonists block the histamine receptors present in these cells (Ash and Schild, 1966; Lind et al., 1983). As a result of anti-secretory therapy, gastric $\mathrm{pH}$ rises. Van Den Abeele et al. measured a mean $\mathrm{pH}$ of $7.4 \pm 0.4$ in gastric aspirates from healthy volunteers after three days of PPI therapy (Van Den Abeele et al., 2015). Since the extent of ionization is crucial for the gastrointestinal solubility of weak bases and acids, significant changes in gastric $\mathrm{pH}$ will impact gastric drug disposition and may have implications for drug bioavailability.

In general, a rise in gastric $\mathrm{pH}$ is disadvantageous for poorly water soluble, weakly basic drugs as the presence of ionized drug in the stomach is reduced and dissolution is hampered, potentially leading to decreased intestinal absorption and bioavailability (Mitra and Kesisoglou, 2013). Walravens et al. determined both gastric and systemic concentrations of posaconazole in healthy volunteers after PPI therapy. The elevation in gastric $\mathrm{pH}$ reduced gastric drug exposure (on average $-84 \%$ ), resulting in a subsequent decrease in systemic posaconazole exposure compared to fasted subjects without prior PPI treatment (on average $-37 \%$ ). As gastric and systemic exposure correlated well, this study emphasizes the importance of including the stomach in studies evaluating gastrointestinal drug disposition of ionizable compounds (Walravens et al., 2011). Similarly, co-administration of antisecretory agents has been shown to hamper absorption of several other weakly basic compounds (e.g. ketoconazole, itraconazole, atazanavir, indinavir and dipyridamole) and some zwitterions (e.g. cefpodoxime and enoxacin) (Chin et al., 1995; Lahner et al., 2009; Tappouni et al., 2008). Contrarily, for nifedipine and digoxin a minor increase in systemic exposure during co-administration with omeprazole was observed in healthy subjects. Inhibition of drug metabolism due to a rise in gastric $\mathrm{pH}$ was ruled out and the 
authors stated that the observed minor interactions of $+10 \%$ and $+26 \%$, respectively, most likely lack clinical relevance (Oosterhuis et al., 1991; Soons et al., 1992). Furthermore, co-administration of bisphosphonate alendronate (zwitterion) with an $\mathrm{H}_{2}$-inhibitor resulted in a two-fold increase in bioavailability. An explanation might be found in the fact that the solubility of sodium alendronate was observed to increase with increasing $\mathrm{pH}$ (Gertz et al., 1995).

Weakly acidic drugs would theoretically benefit from an elevated gastric $\mathrm{pH}$ as gastric drug dissolution increases. Van Den Abeele et al. administered a diclofenac potassium solution to healthy volunteers following a PPI regimen. Concomitant PPI use ensured complete dissolution of diclofenac in the stomach, whereas in the fasted, acidic stomach extensive drug precipitation was observed. The authors hypothesized that transfer of drug from the stomach to the duodenum in a dissolved state compared to as a solid would result in more rapid drug absorption in the former case. However, due to an observed rapid duodenal re-dissolution of gastric drug precipitate in the control condition, it was concluded that PPI intake most likely did not affect systemic exposure of diclofenac. This study suggests that intestinal re-dissolution kinetics of drug precipitated in the stomach will dictate the onset and extent of absorption of weakly acidic compounds (Van Den Abeele et al., 2015).

An increase in gastric $\mathrm{pH}$ may also be disadvantageous for the performance of enteric-coated dosage forms. In this case, dissolution of the enteric coating may already be initiated at the level of the stomach resulting in premature drug release. Surprisingly, little research has been done regarding this interaction, although PPIs and enteric-coated dosage forms are broadly used. Nefesoglu et al. investigated the interaction between omeprazole and enteric-coated salicylate tablets. Omeprazole treatment significantly increased the rate of salicylate absorption from enteric-coated tablets (Nefesoglu et al., 1998).

\subsection{Beverages}

\subsubsection{Beverages - $\mathrm{pH}$}

Although its incidence is unknown, administration of oral dosage forms with other beverages than the intended water may occur in practice, e.g. for taste-masking purposes. Sodas are generally more acidic 
(pH 1.2 - 2.95) compared to tap water (pH 6 - 6.7) (Walravens et al., 2011). Multiple studies have been conducted to investigate the effect of dosage form administration with Coca-Cola ${ }^{\circledR}$ on gastrointestinal drug disposition. For example, Jaruratanasirikul et al. conducted a study to investigate the effect of Coca$\mathrm{Cola}^{\circledR}$ on systemic pharmacokinetics of itraconazole in healthy volunteers. On average, both $\mathrm{C}_{\max }$ and overall systemic exposure increased when the dosage form was ingested with Coca-Cola ${ }^{\circledR}$ compared to ingestion with water (+121 and $+80 \%$, respectively) (Jaruratanasirikul and Kleepkaew, 1997). Using an in vitro/in silico approach, Fotaki et al. corroborated the finding that co-administering itraconazole with Coca-Cola ${ }^{\circledR}$ would result in a higher $\mathrm{C}_{\max }$ and systemic exposure compared to administration with tap water (Fotaki and Klein, 2013). Co-administration of an acidic beverage has also proven to be an effective strategy to increase the systemic exposure of erlotinib, riboflavin and carbamazepine (Houston and Levy, 1975; Malhotra et al., 2002; van Leeuwen et al., 2016). Moreover, several authors have observed that co-administration of Coca-Cola ${ }^{\circledR}$ can partly counter a decrease in bioavailability due to an increase in gastric pH. For instance, Lange et al. observed an increase in itraconazole bioavailability in patients on $\mathrm{H}_{2}$-antagonist therapy when the drug was co-administered with Coca-Cola ${ }^{\circledR}$ (Lange et al., 1997). Similarly, a decrease in both posaconazole and ketoconazole bioavailability in subjects on antisecretory therapy could be partially negated by co-administration with Coca-Cola ${ }^{\circledR}$ (Chin et al., 1995; Walravens et al., 2011). Apart from their well-described effect on intestinal drug metabolism and transport, fruit juices are rather acidic (Bailey et al., 1991; Birkhed, 1984; Greenblatt, 2009; RodríguezFragoso et al., 2011). Consequently, comparable to sodas, fruit juices may influence the degree of ionization of weakly acidic and basic drugs in the stomach and thus their bioavailability.

Apart from the acidity of ingested beverages, other factors may contribute to changes in oral drug disposition and remain to be elucidated. To better understand the impact of oral drug administration with Coca-Cola ${ }^{\circledR}$ on the gastrointestinal and systemic disposition of posaconazole, Walravens et al. performed an in vivo study with healthy volunteers. Although Coca-Cola ${ }^{\circledR}$ did not change the $\mathrm{pH}$ of the already acidic stomach, an increase in gastric and systemic exposure compared to administration with tap water was observed (+102 and $+70 \%$, respectively), confirming earlier findings by Krishna et al (Krishna et al., 2008). The authors attributed these findings to (i) higher solubility of posaconazole in Coca-Cola ${ }^{\circledR}$ 
compared to water and (ii) prolonged gastric residence of the drug due to the caloric density and presence of phosphoric acid in this beverage (Walravens et al., 2011).

\subsubsection{Beverages - temperature}

Ingestion of an oral dosage form with a hot beverage can theoretically lead to an increased solubility of the co-ingested drug. However, this temperature effect will be temporary as the temperature of gastric contents will eventually return to $37^{\circ} \mathrm{C}$. To date, the effects of temperature on oral drug disposition have not been investigated. Sun et al. studied the effects of both hot $\left(50^{\circ} \mathrm{C}\right)$ and cold $\left(4^{\circ} \mathrm{C}\right)$ beverages on changes in gastric content temperature and gastric emptying in healthy volunteers (Sun et al., 1988). Sixty seconds after ingestion of the hot drink, the maximum temperature of gastric content measured $43^{\circ} \mathrm{C}$. On the other hand, minimum temperature of gastric contents was measured 45 seconds after drinking the cold drink and amounted to $21^{\circ} \mathrm{C}$. Intragastric temperature subsequently returned to body temperature within 20 to 30 minutes. Gastric emptying was slower within the first 10 minutes after ingestion of a cold drink compared to a control group, whereas a hot drink did not have a marked effect on gastric emptying compared to the control group (Sun et al., 1988). Bateman et al. corroborated the finding that ingestion of a cold drink $\left(12^{\circ} \mathrm{C}\right)$ initially slows down gastric emptying (Bateman, 1982). However, up to now no consensus regarding the influence of temperature on gastric emptying has been reached, as illustrated by the fact that McArthur et al. observed no difference in gastric emptying after infusing coffee of different temperatures $\left(4^{\circ} \mathrm{C}, 37^{\circ} \mathrm{C}\right.$ and $\left.58^{\circ} \mathrm{C}\right)$ into the stomach of healthy volunteers (McArthur and Feldman, 1989).

\subsubsection{Beverages - alcohol}

Alcohol consumption may affect intragastric formulation disintegration and drug solubility, potentially leading to unexpected systemic exposure (Lennernäs, 2009). By affecting a dosage form's release mechanism, drug release may be enhanced. Accelerated drug release from an enteric-coated or extended-release dosage form may subsequently lead to dose dumping. For example, an extendedrelease capsule containing hydromorphine (Palladone ${ }^{\circledR}$ ) was voluntarily suspended from the market 
once severe ethanol-induced dose dumping was observed in healthy volunteers (FDA, 2005). Ingestion of a single dose of Palladone ${ }^{\circledR}$ with $240 \mathrm{~mL}$ of either a 20 or a $40 \%$ ethanol solution resulted in a 1.89and 5.53-fold increase in mean $\mathrm{C}_{\max }$ of hydromorphine compared to ingestion with water, respectively (Walden et al., 2007). Ever since, a lot of research has been dedicated to identifying formulations susceptible to ethanol-induced dose dumping (Henderson et al., 2007; Sathyan et al., 2007). Johnson et al. investigated the effect of ingesting $240 \mathrm{~mL}$ of $4 \%, 20 \%$ and $40 \%$ of alcohol on the pharmacokinetics of morphine sulfate-naltrexone capsules in healthy volunteers. No significant changes in drug pharmacokinetics were observed when these capsules were co-administered with $4 \%$ and $20 \%$ alcohol, whereas co-administration of $40 \%$ alcohol resulted in a two-fold increase in $\mathrm{C}_{\max }$ and a two-fold reduction in $\mathrm{t}_{\max }$ compared to co-administration with water (Johnson et al., 2012). As a result of these findings, both the US Food and Drug Administration (FDA) and the European Medicines Agency (EMA) drafted guidelines requesting in vitro dissolution tests of formulations potentially at risk in the presence of varying amounts of ethanol (Anand et al., 2011; EMA, 2009; FDA, 2014a). Among other conditions, the FDA requests a dissolution test with $40 \%$ alcohol for two hours, simulating the gastric environment after ingestion of whisky. However, the relevance of this test can be questioned as Rubbens et al. observed an average maximal ethanol concentration of only $11.1 \%$ in the stomach of fasted healthy volunteers after drinking two whiskies $(40 \%, 80 \mathrm{~mL})$. Furthermore, the authors observed a rapid decline in intragastric ethanol concentrations: less than $0.5 \%$ ethanol was present in the stomach after two hours (Rubbens et al., 2016).

Besides the effect of ethanol on dosage form performance, large intragastric ethanol concentration may act as a co-solvent, potentially increasing drug solubility. The extent to which compounds are affected depends on their physicochemical properties. For instance, Fagerberg et al. observed a large ethanolinduced increase in solubility for three neutral compounds in simulated gastric fluids. Two out of three weakly acidic compounds were positively affected as well. The solubility of weak bases was unaffected as these are already ionized in an acidic gastric environment (Fagerberg et al., 2015). Moreover, an ethanol-induced increase in drug solubility may lead to changes in drug release mechanism from erosion-mediated to diffusion-mediated drug release (Roberts et al., 2007). 


\section{Concluding remarks}

The stomach is a complex and variable environment, subject to dynamic changes based on a multitude of stimuli, e.g. prandial state, population-related differences and the presence of an underlying pathology. As a result, the stomach may substantially contribute to the inter-individual variability often observed for orally administered drug delivery systems. Adding to this variability in drug disposition are the so-called real-life dosing conditions, which may alter physiological parameters and, in doing so, may influence gastrointestinal drug disposition. From a drug development point of view, it may therefore be wise to further map this variability in order to ensure adequate drug performance, even under extreme, 'abnormal' conditions by performing high-quality experiments in large study populations and by including real-life dosing conditions. Suffice it to say, the stomach should no longer be considered as a mere waiting room for a drug to be absorbed but better be regarded as a compartment which may markedly benefit and/or harm drug disposition and subsequent systemic exposure. Therefore, it is recommended to consider the impact of a drug's gastric passage during drug development by including relevant gastric parameters (acidity, motility) in predictive in vitro setups, depending on drug properties (e.g. susceptibility to acid degradation, weakly acidic/basic properties).

\section{Acknowledgements}

This work has received support from the Research Foundation - Flanders (FWO) (PhD fellowship $11 \mathrm{Z} 2615 \mathrm{~N}$ and Research grant No. G.0769.14N) and the Innovative Medicines Initiative Joint Undertaking (http://www.imi.europa.eu) under Grant Agreement No. 115369, resources of which are composed of financial contribution from the European Union's Seventh Framework Program and EFPIA companies in kind contribution.

Figures 1, 2, 3 and 5 were produced using Servier Medical Art (http://www.servier.com/Powerpointimage-bank). 


\section{References}

Abrahamsson, B., Alpsten, M., Jonsson, U.E., Lundberg, P.J., Sandberg, A., Sundgren, M., Svenheden, A., Tölli, J., 1996. Gastro-intestinal transit of a multiple-unit formulation (metoprolol CR/ZOK) and a non-disintegrating tablet with the emphasis on colon. International journal of pharmaceutics 140,229 235.

Abrahamsson, B., Alpsten, M., Bake, B., Jonsson, U.E., Eriksson-Lepkowska, M., Larsson, A., 1998. Drug absorption from nifedipine hydrophilic matrix extended-release (ER) tablet-comparison with an osmotic pump tablet and effect of food. Journal of Controlled Release 52, 301-310.

Abrahamsson, B., Roos, K., Sjögren, J., 1999. Investigation of prandial effects on hydrophilic matrix tablets. Drug development and industrial pharmacy 25, 765-771.

Abrahamsson, B., Pal, A., Sjoberg, M., Carlsson, M., Laurell, E., Brasseur, J.G., 2005. A novel in vitro and numerical analysis of shear-induced drug release from extended-release tablets in the fed stomach. Pharmaceutical research 22, 1215-1226.

al-Janabi, J., Hartsuck, J.A., Tang, J., 1972. Kinetics and mechanism of pepsinogen activation. The Journal of biological chemistry 247, 4628-4632.

Al-Marhoon, M.S., Nunn, S., Soames, R.W., 2005. Effects of cagA+ and cagA- strains of Helicobacter pylori on the human gastric mucus layer thickness. Journal of gastroenterology and hepatology 20, 1246-1252.

Allen, A., Flemstrom, G., 2005. Gastroduodenal mucus bicarbonate barrier: protection against acid and pepsin. American journal of physiology. Cell physiology 288, C1-19.

Alvis, B.D., Hughes, C.G., 2015. Physiology Considerations in Geriatric Patients. Anesthesiology clinics 33, 447-456.

Anand, O., Yu, L.X., Conner, D.P., Davit, B.M., 2011. Dissolution testing for generic drugs: an FDA perspective. The AAPS journal 13, 328-335.

Anderson, B.J., van Lingen, R.A., Hansen, T.G., Lin, Y.C., Holford, N.H., 2002. Acetaminophen developmental pharmacokinetics in premature neonates and infants: a pooled population analysis. Anesthesiology 96, 1336-1345.

Angrisani, L., Santonicola, A., Iovino, P., Formisano, G., Buchwald, H., Scopinaro, N., 2015. Bariatric Surgery Worldwide 2013. Obesity surgery 25, 1822-1832.

Armand, M., Borel, P., Pasquier, B., Dubois, C., Senft, M., Andre, M., Peyrot, J., Salducci, J., Lairon, D., 1996a. Physicochemical characteristics of emulsions during fat digestion in human stomach and duodenum. The American journal of physiology 271, G172-183.

Armand, M., Hamosh, M., Mehta, N.R., Angelus, P.A., Philpott, J.R., Henderson, T.R., Dwyer, N.K., Lairon, D., Hamosh, P., 1996b. Effect of human milk or formula on gastric function and fat digestion in the premature infant. Pediatric research 40, 429-437.

Armand, M., Pasquier, B., Andre, M., Borel, P., Senft, M., Peyrot, J., Salducci, J., Portugal, H., Jaussan, V., Lairon, D., 1999. Digestion and absorption of 2 fat emulsions with different droplet sizes in the human digestive tract. The American journal of clinical nutrition 70, 1096-1106.

Armand, M., 2007. Lipases and lipolysis in the human digestive tract: where do we stand? Current opinion in clinical nutrition and metabolic care 10, 156-164.

Arora, S., Ali, J., Ahuja, A., Khar, R.K., Baboota, S., 2005. Floating drug delivery systems: A review. AAPS PharmSciTech 6, E372-E390.

Ash, A.S.F., Schild, H.O., 1966. Receptors mediating some actions of histamine. British Journal of Pharmacology and Chemotherapy 27, 427-439.

Avery, G.B., Randolph, J.G., Weaver, T., 1966. Gastric acidity in the first day of life. Pediatrics 37, 10051007.

Bailey, D.G., Spence, J.D., Munoz, C., Arnold, J.M., 1991. Interaction of citrus juices with felodipine and nifedipine. Lancet 337, 268-269.

Bakala-N'Goma, J.C., Williams, H.D., Sassene, P.J., Kleberg, K., Calderone, M., Jannin, V., Igonin, A., Partheil, A., Marchaud, D., Jule, E., Vertommen, J., Maio, M., Blundell, R., Benameur, H., Mullertz, A., 
Pouton, C.W., Porter, C.J., Carriere, F., 2015. Toward the establishment of standardized in vitro tests for lipid-based formulations. 5. Lipolysis of representative formulations by gastric lipase. Pharmaceutical research 32, 1279-1287.

Balasubramanian, K., Zentler-Munro, P.L., Batten, J.C., Northfield, T.C., 1992. Increased intragastric acid-resistant lipase activity and lipolysis in pancreatic steatorrhoea due to cystic fibrosis. Pancreas 7 , 305-310.

Banerjee, S., Dixit, S., Fox, M., Pal, A., 2014. Validation of a rapid, semiautomatic image analysis tool for measurement of gastric accommodation and emptying by magnetic resonance imaging. American Journal of Physiology - Gastrointestinal and Liver Physiology 308, 652-663.

Baron, J.H., 1969. Lean body mass, gastric acid, and peptic ulcer. Gut 10, 637-642.

Bash, E., Troy, D., 2015. Remington the science and practice of pharmacy. PhD Proposal 1.

Bass, 1993. Gastric Emptying; Differences among Liquid, Fiber, Polymer and Solid Dosage Forms of Medications. Capsugel Symposium Series, 21-33.

Bateman, D.N., 1982. Effects of meal temperature and volume on the emptying of liquid from the human stomach. The Journal of physiology 331, 461-467.

Becker, D., Zhang, J., Heimbach, T., Penland, R.C., Wanke, C., Shimizu, J., Kulmatycki, K., 2014. Novel Orally Swallowable IntelliCap ${ }^{\circledR}$ Device to Quantify Regional Drug Absorption in Human GI Tract Using Diltiazem as Model Drug. AAPS PharmSciTech 15, 1490-1497.

Bennink, R., Peeters, M., Van den Maegdenbergh, V., Geypens, B., Rutgeerts, P., De Roo, M., Mortelmans, L., 1998. Comparison of total and compartmental gastric emptying and antral motility between healthy men and women. European journal of nuclear medicine 25, 1293-1299.

Bevernage, J., Hens, B., Brouwers, J., Tack, J., Annaert, P., Augustijns, P., 2012. Supersaturation in human gastric fluids. European journal of pharmaceutics and biopharmaceutics 81, 184-189.

Birkhed, D., 1984. Sugar content, acidity and effect on plaque $\mathrm{pH}$ of fruit juices, fruit drinks, carbonated beverages and sport drinks. Caries research 18, 120-127.

Bjerrum, L., Sogaard, J., Hallas, J., Kragstrup, J., 1998. Polypharmacy: correlations with sex, age and drug regimen. A prescription database study. European journal of clinical pharmacology 54, 197-202.

Blaha, J.M., Knevel, a.M., Kessler, D.P., Mincy, J.W., Hem, S.L., 1976. Kinetic analysis of penicillin degradation in acidic media. Journal of pharmaceutical sciences 65, 1165-1170.

Blumenthal, I., Pildes, R.S., 1979. Effect of posture on the pattern of stomach emptying in the newborn. Pediatrics 63, 532-536.

Bolondi, L., Bortolotti, M., Santi, V., Calletti, T., Gaiani, S., Labò, G., 1985. Measurement of gastric emptying time by real-time ultrasonography. Gastroenterology 89, 752-759.

Borgstrom, B., Dahlqvist, A., Lundh, G., Sjovall, J., 1957. Studies of intestinal digestion and absorption in the human. The Journal of clinical investigation 36, 1521-1536.

Bortolotti, M., Annese, V., Coccia, G., 2000. Twenty-four hour ambulatory antroduodenal manometry in normal subjects (co-operative study). Neurogastroenterology and motility 12, 231-238.

Boulby, P., Moore, R., Gowland, P., Spiller, R.C., 1999. Fat delays emptying but increases forward and backward antral flow as assessed by flow-sensitive magnetic resonance imaging. Neurogastroenterology and motility 11, 27-36.

Bouras, E.P., 2002. SPECT imaging of the stomach: comparison with barostat, and effects of sex, age, body mass index, and fundoplication. Gut 51, 781-786.

Bourlieu, C., Menard, O., Bouzerzour, K., Mandalari, G., Macierzanka, A., Mackie, A.R., Dupont, D., 2014. Specificity of infant digestive conditions: some clues for developing relevant in vitro models. Critical reviews in food science and nutrition 54, 1427-1457.

Braghetto, I., Davanzo, C., Korn, O., Csendes, A., Valladares, H., Herrera, E., Gonzalez, P., Papapietro, K., 2009. Scintigraphic evaluation of gastric emptying in obese patients submitted to sleeve gastrectomy compared to normal subjects. Obesity surgery 19, 1515-1521.

Brancato, D., Scorsone, A., Saura, G., Ferrara, L., Di Noto, A., Aiello, V., Fleres, M., Provenzano, V., 2014. Comparison of TSH Levels with Liquid Formulation Versus Tablet Formulations of Levothyroxine in the Treatment of Adult Hypothyroidism. Endocrine practice 20, 657-662. 
Bredenoord, A.J., Smout, A.J.P.M., 2008. High-resolution manometry. Digestive and Liver Disease 40, 174-181.

Brennan, I.M., Feltrin, K.L., Nair, N.S., Hausken, T., Little, T.J., Gentilcore, D., Wishart, J.M., Jones, K.L., Horowitz, M., Feinle-Bisset, C., 2009. Effects of the phases of the menstrual cycle on gastric emptying, glycemia, plasma GLP-1 and insulin, and energy intake in healthy lean women. American journal of physiology - Gastrointestinal and liver physiology 297, G602-610.

Brouwers, J., Tack, J., Augustijns, P., 2007. Parallel Monitoring of Plasma and Intraluminal Drug Concentrations in Man After Oral Administration of Fosamprenavir in the Fasted and Fed State. Pharmaceutical research 24, 1862-1869.

Brouwers, J., Brewster, M.E., Augustijns, P., 2009. Supersaturating drug delivery systems: The answer to solubility-limited oral bioavailability? Journal of pharmaceutical sciences 98, 2549-2572.

Brouwers, J., Anneveld, B., Goudappel, G.-J., Duchateau, G., Annaert, P., Augustijns, P., Zeijdner, E., 2011. Food-dependent disintegration of immediate release fosamprenavir tablets: In vitro evaluation using magnetic resonance imaging and a dynamic gastrointestinal system. European Journal of Pharmaceutics and Biopharmaceutics 77, 313-319.

Burdan, F., Rozylo-Kalinowska, I., Szumilo, J., Zinkiewicz, K., Dworzanski, W., Krupski, W., Dabrowski, A., 2012. Anatomical classification of the shape and topography of the stomach. Surgical and radiologic anatomy : SRA 34, 171-178.

Caballero-Plasencia, A.M., Valenzuela-Barranco, M., Martin-Ruiz, J.L., Herrerias-Gutierrez, J.M., Esteban-Carretero, J.M., 1999. Are there changes in gastric emptying during the menstrual cycle? Scandinavian journal of gastroenterology 34, 772-776.

Calbet, J.A., MacLean, D.A., 1997. Role of caloric content on gastric emptying in humans. The Journal of physiology 498 (Pt 2), 553-559.

Camilleri, M., Malagelada, J.R., Brown, M.L., Becker, G., Zinsmeister, A.R., 1985. Relation between antral motility and gastric emptying of solids and liquids in humans. The American journal of physiology 249, G580-585.

Camilleri, M., Bharucha, A.E., Di lorenzo, C., Hasler, W.L., Prather, C.M., Rao, S.S., Wald, A., 2008. American Neurogastroenterology and Motility Society consensus statement on intraluminal measurement of gastrointestinal and colonic motility in clinical practice. Neurogastroenterology \& Motility 20, 1269-1282.

Carlos, M.A., Babyn, P.S., Marcon, M.A., Moore, A.M., 1997. Changes in gastric emptying in early postnatal life. The Journal of pediatrics 130, 931-937.

Carriere, F., Renou, C., Lopez, V., De Caro, J., Ferrato, F., Lengsfeld, H., De Caro, A., Laugier, R., Verger, R., 2000. The specific activities of human digestive lipases measured from the in vivo and in vitro lipolysis of test meals. Gastroenterology 119, 949-960.

Carriere, F., Renou, C., Ransac, S., Lopez, V., De Caro, J., Ferrato, F., De Caro, A., Fleury, A., SanwaldDucray, P., Lengsfeld, H., Beglinger, C., Hadvary, P., Verger, R., Laugier, R., 2001. Inhibition of gastrointestinal lipolysis by Orlistat during digestion of test meals in healthy volunteers. American journal of physiology. Gastrointestinal and liver physiology 281, G16-28.

Carriere, F., Grandval, P., Renou, C., Palomba, A., Prieri, F., Giallo, J., Henniges, F., Sander-Struckmeier, S., Laugier, R., 2005. Quantitative study of digestive enzyme secretion and gastrointestinal lipolysis in chronic pancreatitis. Clinical gastroenterology and hepatology 3, 28-38.

Cassilly, D., Kantor, S., Knight, L.C., Maurer, A.H., Fisher, R.S., Semler, J., Parkman, H.P., 2008. Gastric emptying of a non-digestible solid: assessment with simultaneous SmartPill pH and pressure capsule, antroduodenal manometry, gastric emptying scintigraphy. Neurogastroenterology and motility 20, 311-319.

Castro-Combs, J., Garcia, C.J., Majewski, M., Wallner, G., Sarosiek, J., 2014. Impaired viscosity of gastric secretion and its mucin content as potential contributing factors to the development of chronic constipation. Digestive diseases and sciences 59, 2730-2734.

Celli, J.P., Turner, B.S., Afdhal, N.H., Keates, S., Ghiran, I., Kelly, C.P., Ewoldt, R.H., McKinley, G.H., So, P., Erramilli, S., Bansil, R., 2009. Helicobacter pylori moves through mucus by reducing mucin 
viscoelasticity. Proceedings of the National Academy of Sciences of the United States of America 106, 14321-14326.

Charman, W.N., Porter, C.J., Mithani, S., Dressman, J.B., 1997. Physiochemical and physiological mechanisms for the effects of food on drug absorption: the role of lipids and $\mathrm{pH}$. Journal of pharmaceutical sciences 86, 269-282.

Chaw, C.S., Yazaki, E., Evans, D.F., 2001. The effect of pH change on the gastric emptying of liquids measured by electrical impedance tomography and $\mathrm{pH}$-sensitive radiotelemetry capsule. International journal of pharmaceutics 227, 167-175.

Chen, C., Bujanover, S., Kareht, S., Rapoport, A.M., 2015. Differential pharmacokinetics of diclofenac potassium for oral solution vs immediate-release tablets from a randomized trial: effect of fed and fasting conditions. Headache 55, 265-275.

Chen, J.D., Co, E., Liang, J., Pan, J., Sutphen, J., Torres-Pinedo, R.B., Orr, W.C., 1997. Patterns of gastric myoelectrical activity in human subjects of different ages. The American journal of physiology 272 , $1022-1027$.

Chen, T.F., Yadav, P.K., Wu, R.J., Yu, W.H., Liu, C.Q., Lin, H., Liu, Z.J., 2013. Comparative evaluation of intragastric bile acids and hepatobiliary scintigraphy in the diagnosis of duodenogastric reflux. World journal of gastroenterology 19, 2187-2196.

Chessa, S., Huatan, H., Levina, M., Mehta, R.Y., Ferrizzi, D., Rajabi-Siahboomi, A.R., 2014. Application of the Dynamic Gastric Model to evaluate the effect of food on the drug release characteristics of a hydrophilic matrix formulation. International journal of pharmaceutics 466, 359-367.

Chin, T.W., Loeb, M., Fong, I.W., 1995. Effects of an acidic beverage (Coca-Cola) on absorption of ketoconazole. Antimicrobial Agents and Chemotherapy 39, 1671-1675.

CHMP, 2010. Guideline on the investigation of bioequivlance.

Chourasia, M.K., Jain, S.K., 2004. Polysaccharides for Colon Targeted Drug Delivery. Drug delivery 11, 129-148.

Chu, S., Schubert, M.L., 2013. Gastric secretion. Current opinion in gastroenterology 29, 636-641.

Clarkston, W.K., Pantano, M.M., Morley, J.E., Horowitz, M., Littlefield, J.M., Burton, F.R., 1997.

Evidence for the anorexia of aging: gastrointestinal transit and hunger in healthy elderly vs. young adults. The American journal of physiology 272, R243-248.

Collins, P.J., Horowitz, M., Maddox, A., Myers, J.C., Chatterton, B.E., 1996. Effects of increasing solid component size of a mixed solid/liquid meal on solid and liquid gastric emptying. The American journal of physiology 271, G549-554.

Cone, R.A., 2009. Barrier properties of mucus. Advanced drug delivery reviews 61, 75-85.

Conklin, J., 2009. Gastric/Small Bowel Manometry, in: Soffer, E., Pimentel, M., Conklin, J. (Eds.), Color Atlas of High Resolution Manometry. Springer US, Boston, MA, pp. 59-70.

Conley, R., Gupta, S.K., Sathyan, G., 2006. Clinical spectrum of the osmotic-controlled release oral delivery system (OROS), an advanced oral delivery form. Current medical research and opinion 22, 1879-1892.

Cordova-Fraga, T., 2008. Effects of anatomical position on esophageal transit time: A biomagnetic diagnostic technique. World Journal of Gastroenterology 14, 5707.

Cortot, A., Colombel, J., 1984. Gastric emptying of OROS tablets in man. International journal of pharmaceutics 22, 321-325.

Coupe, A.J., Davis, S.S., Evans, D.F., Wilding, I.R., 1991. Correlation of the Gastric Emptying of Nondisintegrating Tablets with Gastrointestinal Motility. Pharmaceutical research 8, 1281-1285.

Crane, C.W., Luntz, G.R., 1968. Absorption of insulin from the human small intestine. Diabetes 17, 625627.

Cunningham, K.M., Horowitz, M., Riddell, P.S., Maddern, G.J., Myers, J.C., Holloway, R.H., Wishart, J.M., Jamieson, G.G., 1991. Relations among autonomic nerve dysfunction, oesophageal motility, and gastric emptying in gastro-oesophageal reflux disease. Gut 32, 1436-1440.

Dai, F., Gong, J., Zhang, R., Luo, J.Y., Zhu, Y.L., Wang, X.Q., 2002. Assessment of duodenogastric reflux by combined continuous intragastric $\mathrm{pH}$ and bilirubin monitoring. World journal of gastroenterology 8, 382-384. 
Dalenback, J., Mellander, A., Olbe, L., Sjovall, H., 1993. Motility-related cyclic fluctuations of interdigestive gastric acid and bicarbonate secretion in man. A source of substantial variability in gastric secretion studies. Scandinavian journal of gastroenterology 28, 943-948.

Davis, S.S., Stockwell, A.F., Taylor, M.J., Hardy, J.G., Whalley, D.R., Wilson, C.G., Bechgaard, H., Christensen, F.N., 1986. The effect of density on the gastric emptying of single- and multiple-unit dosage forms. Pharmaceutical research 3, 208-213.

Davis, S.S., Hardy, J.G., Newman, S.P., Wilding, I.R., 1992. Gamma scintigraphy in the evaluation of pharmaceutical dosage forms. European journal of nuclear medicine 19.

De Schepper, H., Camilleri, M., Cremonini, F., Foxx-Orenstein, A., Burton, D., 2004. Comparison of gastric volumes in response to isocaloric liquid and mixed meals in humans. Neurogastroenterology and motility 16, 567-573.

De Smet, J., Van Bocxlaer, J., Boussery, K., 2013. The influence of bypass procedures and other anatomical changes in the gastrointestinal tract on the oral bioavailability of drugs. Journal of clinical pharmacology 53, 361-376.

Debotton, N., Dahan, A., 2014. A mechanistic approach to understanding oral drug absorption in pediatrics: an overview of fundamentals. Drug discovery today 19, 1322-1336.

Deloose, E., Janssen, P., Depoortere, I., Tack, J., 2012. The migrating motor complex: control mechanisms and its role in health and disease. Nature reviews - Gastroenterology \& hepatology 9, 271285.

Desipio, J., Friedenberg, F.K., Korimilli, a., Richter, J.E., Parkman, H.P., Fisher, R.S., 2007. Highresolution solid-state manometry of the antropyloroduodenal region. Neurogastroenterology and Motility 19, 188-195.

Dewar, P., King, R., Johnston, D., 1982. Bile acid and lysolecithin concentrations in the stomach in patients with duodenal ulcer before operation and after treatment by highly selective vagotomy, partial gastrectomy, or truncal vagotomy and drainage. Gut 23, 569-577.

Di Ciaula, A., Wang, D.Q., Portincasa, P., 2012. Gallbladder and gastric motility in obese newborns, preadolescents and adults. Journal of gastroenterology and hepatology 27, 1298-1305.

Diakidou, A., Vertzoni, M., Dressman, J., Reppas, C., 2009. Estimation of intragastric drug solubility in the fed state: comparison of various media with data in aspirates. Biopharmaceutics \& drug disposition 30, 318-325.

Digenis, G.A., Sandefer, E.P., Parr, A.F., Beihn, R., McClain, C., Scheinthal, B.M., Ghebre-Sellassie, I., lyer, U., Nesbitt, R.U., Randinitis, E., 1990. Gastrointestinal Behavior of Orally Administered Radiolabeled Erythromycin Pellets in Man as Determined by Gamma Scintigraphy. The Journal of Clinical Pharmacology 30, 621-631.

DiPalma, J., Kirk, C.L., Hamosh, M., Colon, A.R., Benjamin, S.B., Hamosh, P., 1991. Lipase and pepsin activity in the gastric mucosa of infants, children, and adults. Gastroenterology 101, 116-121.

Distrutti, E., Azpiroz, F., Soldevilla, A., Malagelada, J.R., 1999. Gastric wall tension determines perception of gastric distention. Gastroenterology 116, 1035-1042.

Dooley, C.P., Di Lorenzo, C., Valenzuela, J.E., 1992. Variability of migrating motor complex in humans. Digestive diseases and sciences 37, 723-728.

Dressman, J.B., Berardi, R.R., Dermentzoglou, L.C., Russell, T.L., Schmaltz, S.P., Barnett, J.L., Jarvenpaa, K.M., 1990. Upper gastrointestinal (GI) pH in young, healthy men and women. Pharmaceutical research 7, 756-761.

Dubois, A., Eerdewegh, P.V., Gardner, J.D., 1977. Gastric emptying and secretion in Zollinger-Ellison syndrome. The Journal of clinical investigation 59, 255-263.

Dwivedi, S., Kumar, V., 2011. Floating Drug Delivery Systems- A Concept of Gastroretention Dosages Form. International Journal of Research in Pharmaceutical and Biomedical Sciences 2, 1413-1426.

Ebers, D.W., Gibbs, G.E., Smith, D.I., 1956. Gastric acidity on the first day of life. Pediatrics 18, 800-802. Efentakis, M., Dressman, J.B., 1998. Gastric juice as a dissolution medium: surface tension and pH. European journal of drug metabolism and pharmacokinetics 23, 97-102.

Eisen, G.M., Eliakim, R., Zaman, A., Schwartz, J., Faigel, D., Rondonotti, E., Villa, F., Weizman, E., Yassin, K., deFranchis, R., 2006. The Accuracy of PillCam ESO Capsule Endoscopy Versus Conventional Upper 
Endoscopy for the Diagnosis of Esophageal Varices: A Prospective Three-Center Pilot Study. Endoscopy 38, 31-35.

Ellis, H., 2011. Anatomy of the stomach. Surgery (Oxford) 29, 541-543.

EMA, 2009. Quality of medicines questions and answers. European Medicines Agency.

Ensign, L.M., Cone, R., Hanes, J., 2012. Oral drug delivery with polymeric nanoparticles: the gastrointestinal mucus barriers. Advanced drug delivery reviews 64, 557-570.

Fagerberg, J.H., Sjögren, E., Bergström, C.A.S., 2015. Concomitant intake of alcohol may increase the absorption of poorly soluble drugs. European Journal of Pharmaceutical Sciences 67, 12-20.

Fallingborg, J., Christensen, L.A., Ingeman-Nielsen, M., Jacobsen, B.A., Abildgaard, K., Rasmussen, H.H., Rasmussen, S.N., 1990. Measurement of gastrointestinal $\mathrm{pH}$ and regional transit times in normal children. Journal of pediatric gastroenterology and nutrition 11, 211-214.

Farmer, A.D., Scott, S.M., Hobson, A.R., 2013. Gastrointestinal motility revisited: The wireless motility capsule. United European Gastroenterology Journal 1, 413-421.

Farre, R., Tack, J., 2013. Food and symptom generation in functional gastrointestinal disorders: physiological aspects. The American journal of gastroenterology 108, 698-706.

FDA, 2005. FDA alert for healthcare professionals: hydromorphone hydrochloride extended-release capsules (marketed as Palladone). Food and Drug Aministration.

FDA, 2014a. Draft Guidance on Hydromorphone Hydrochloride. Food and Drug Administration.

FDA, 2014b. Guidance for Industry: Bioavailability and Bioequivalence Studies Submitted in NDAs or INDs - General Considerations (draft guidance). Food and Drug Administration.

Feldman, M., Richardson, C.T., Walsh, J.H., 1983. Sex-related differences in gastrin release and parietal cell sensitivity to gastrin in healthy human beings. The Journal of clinical investigation 71, 715-720.

Feldman, M., Cryer, B., McArthur, K.E., Huet, B.A., Lee, E., 1996. Effects of aging and gastritis on gastric acid and pepsin secretion in humans: a prospective study. Gastroenterology 110, 1043-1052.

Felton, L.a., 2011. Oral Controlled Release Formulation Design and Drug Delivery: Theory to Practice. 363.

Ferrua, M.J., Singh, R.P., 2010. Modeling the fluid dynamics in a human stomach to gain insight of food digestion. Journal of food science 75, R151-162.

Fiese, E.F., Steffen, S.H., 1990. Comparison of the acid stability of azithromycin and erythromycin A. The Journal of antimicrobial chemotherapy 25 Suppl A, 39-47.

Finholt, P., Juergensen, G., Kristiansen, H., 1965. Catalytic Effect of Buffers on Degradation of Penicillin $G$ in Aqueous Solution. Journal of pharmaceutical sciences 54, 387-393.

Foltz, E., Azad, S., Everett, M.L., Holzknecht, Z.E., Sanders, N.L., Thompson, J.W., Dubois, L.G., Parker, W., Keshavjee, S., Palmer, S.M., Davis, R.D., Lin, S.S., 2015. An assessment of human gastric fluid composition as a function of PPI usage. Physiological reports 3.

Fonte, P., Araújo, F., Reis, S., Sarmento, B., 2013. Oral Insulin Delivery: How Far Are We? Journal of Diabetes Science and Technology 7, 520-531.

Fotaki, N., Klein, S., 2013. Mechanistic Understanding of the Effect of PPIs and Acidic Carbonated Beverages on the Oral Absorption of Itraconazole Based on Absorption Modeling with Appropriate in Vitro Data. Mol. Pharmaceutics 10, 4016-4023.

Fruehauf, H., Menne, D., Kwiatek, M.A., Forras-Kaufman, Z., Kaufman, E., Goetze, O., Fried, M., Schwizer, W., Fox, M., 2011. Inter-observer reproducibility and analysis of gastric volume measurements and gastric emptying assessed with magnetic resonance imaging. Neurogastroenterology and motility 23, 854-861.

Furness, J.B., 2006. The organisation of the autonomic nervous system: peripheral connections. Autonomic neuroscience : basic \& clinical 130, 1-5.

Garbacz, G., Wedemeyer, R.-S., Nagel, S., Giessmann, T., Mönnikes, H., Wilson, C.G., Siegmund, W., Weitschies, W., 2008. Irregular absorption profiles observed from diclofenac extended release tablets can be predicted using a dissolution test apparatus that mimics in vivo physical stresses. European Journal of Pharmaceutics and Biopharmaceutics 70, 421-428.

Garbacz, G., Golke, B., Wedemeyer, R.S., Axell, M., Soderlind, E., Abrahamsson, B., Weitschies, W., 2009. Comparison of dissolution profiles obtained from nifedipine extended release once a day 
products using different dissolution test apparatuses. European journal of pharmaceutical sciences 38 , 147-155.

Gertz, B.J., Holland, S.D., Kline, W.F., Matuszewski, B.K., Freeman, a., Quan, H., Lasseter, K.C., Mucklow, J.C., Porras, a.G., 1995. Studies of the oral bioavailability of alendronate. Clinical pharmacology and therapeutics 58, 288-298.

Gharpure, V., Meert, K.L., Sarnaik, A.P., Metheny, N.A., 2000. Indicators of postpyloric feeding tube placement in children. Critical care medicine 28, 2962-2966.

Ghoos, Y.F., Maes, B.D., Geypens, B.J., Mys, G., Hiele, M.I., Rutgeerts, P.J., Vantrappen, G., 1993. Measurement of gastric emptying rate of solids by means of a carbon-labeled octanoic acid breath test. Gastroenterology 104, 1640-1647.

Goetze, O., Steingoetter, A., Menne, D., van der Voort, I.R., Kwiatek, M.A., Boesiger, P., Weishaupt, D., Thumshirn, M., Fried, M., Schwizer, W., 2006. The effect of macronutrients on gastric volume responses and gastric emptying in humans: a magnetic resonance imaging study. American Journal of Physiology - Gastrointestinal and Liver Physiology 292, 11-17.

Goetze, O., Treier, R., Fox, M., Steingoetter, A., Fried, M., Boesiger, P., Schwizer, W., 2009. The effect of gastric secretion on gastric physiology and emptying in the fasted and fed state assessed by magnetic resonance imaging. Neurogastroenterology and motility 21, 725-742.

Gonzalez, A., Mugueta, C., Parra, D., Labayen, I., Martinez, A., Varo, N., Monreal, I., Gil, M.J., 2000. Characterisation with stable isotopes of the presence of a lag phase in the gastric emptying of liquids. European journal of nutrition 39, 224-228.

Graham, D.Y., Lacey Smith, J., Bouvet, A.a., 1990. What Happens to Tablets and Capsules in the Stomach: Endoscopic Comparison of Disintegration and Dispersion Characteristics of Two Microencapsulated Potassium Formulations. Journal of pharmaceutical sciences 79, 420-424.

Graham, D.Y., Opekun, A.R., Willingham, F.F., Qureshi, W.A., 2005. Visible small-intestinal mucosal injury in chronic NSAID users. Clinical Gastroenterology and Hepatology 3, 55-59.

Greenblatt, D.J., 2009. Analysis of drug interactions involving fruit beverages and organic aniontransporting polypeptides. Journal of clinical pharmacology 49, 1403-1407.

Greenblatt, H.K., Greenblatt, D.J., 2015. Altered drug disposition following bariatric surgery: a research challenge. Clinical pharmacokinetics 54, 573-579.

Gritti, I., Banfi, G., Roi, G.S., 2000. Pepsinogens: physiology, pharmacology pathophysiology and exercise. Pharmacological research 41, 265-281.

Gupta, M., Brans, Y.W., 1978. Gastric retention in neonates. Pediatrics 62, 26-29.

Gutierrez, M.M., Abramowitz, W., 2000. Pharmacokinetic comparison of oral solution and tablet formulations of citalopram: a single-dose, randomized, crossover study. Clinical therapeutics 22, 15251532.

Haastrup, P., Paulsen, M.S., Zwisler, J.E., Begtrup, L.M., Hansen, J.M., Rasmussen, S., Jarbol, D.E., 2014. Rapidly increasing prescribing of proton pump inhibitors in primary care despite interventions: a nationwide observational study. The European journal of general practice 20, 290-293.

Hamad, G.G., Helsel, J.C., Perel, J.M., Kozak, G.M., McShea, M.C., Hughes, C., Confer, A.L., Sit, D.K., McCloskey, C.A., Wisner, K.L., 2012. The effect of gastric bypass on the pharmacokinetics of serotonin reuptake inhibitors. The American journal of psychiatry 169, 256-263.

Haruma, K., Kamada, T., Kawaguchi, H., Okamoto, S., Yoshihara, M., Sumii, K., Inoue, M., Kishimoto, S., Kajiyama, G., Miyoshi, A., 2000. Effect of age and Helicobacter pylori infection on gastric acid secretion. Journal of gastroenterology and hepatology 15, 277-283.

Hasler, W.L., 2009. The Physiology of Gastric Motility and Gastric Emptying, Textbook of Gastroenterology. Blackwell Publishing Ltd., pp. 207-230.

Heading, R.C., Nimmo, J., Prescott, L.F., Tothill, P., 1973. The dependence of paracetamol absorption on the rate of gastric emptying. British Journal of Pharmacology 47, 415-421.

Heddle, R., Collins, P.J., Dent, J., Horowitz, M., Read, N.W., Chatterton, B., Houghton, L.A., 1989. Motor mechanisms associated with slowing of the gastric emptying of a solid meal by an intraduodenal lipid infusion. Journal of gastroenterology and hepatology 4, 437-447. 
Heetun, Z.S., Quigley, E.M., 2012. Gastroparesis and Parkinson's disease: a systematic review. Parkinsonism \& related disorders 18, 433-440.

Heidelbaugh, J.J., Kim, A.H., Chang, R., Walker, P.C., 2012. Overutilization of proton-pump inhibitors: what the clinician needs to know. Therapeutic Advances in Gastroenterology 5, 219-232.

Hendeles, L., Weinberger, M., Milavetz, G., Hill, M., Vaughan, L., 1985. Food-induced 'dose-dumping' from a once-a-day theophylline product as a cause of theophylline toxicity. Chest 87, 758-765.

Henderson, L.S., Tenero, D.M., Campanile, A.M., Baidoo, C.a., Danoff, T.M., 2007. Ethanol does not alter the pharmacokinetic profile of the controlled-release formulation of carvedilol. Journal of clinical pharmacology 47, 1358-1365.

Hens, B., Brouwers, J., Anneveld, B., Corsetti, M., Symillides, M., Vertzoni, M., Reppas, C., Turner, D.B., Augustijns, P., 2014. Gastrointestinal transfer: in vivo evaluation and implementation in in vitro and in silico predictive tools. European journal of pharmaceutical sciences 63, 233-242.

Hens, B., Brouwers, J., Corsetti, M., Augustijns, P., 2016a. Supersaturation and Precipitation of Posaconazole Upon Entry in the Upper Small Intestine in Humans. Journal of pharmaceutical sciences. Hens, B., Corsetti, M., Brouwers, J., Augustijns, P., 2016b. Gastrointestinal and Systemic Monitoring of Posaconazole in Humans After Fasted and Fed State Administration of a Solid Dispersion. Journal of pharmaceutical sciences.

Hermann, R., Wildgrube, H.J., Ruus, P., Niebch, G., Nowak, H., Gleiter, C.H., 1998. Gastric emptying in patients with insulin dependent diabetes mellitus and bioavailability of thioctic acid-enantiomers. European journal of pharmaceutical sciences 6, 27-37.

Hermansson, G., Sivertsson, R., 1996. Gender-related differences in gastric emptying rate of solid meals. Digestive diseases and sciences 41, 1994-1998.

Hey, H., Matzen, P., Andersen, J.T., Didriksen, E., Nielsen, B., 1979. A gastroscopic and pharmacological study of the disintegration time and absorption of pivampicillin capsules and tablets. British journal of clinical pharmacology 8, 237-242.

Hoad, C.L., Parker, H., Hudders, N., Costigan, C., Cox, E.F., Perkins, A.C., Blackshaw, P.E., Marciani, L., Spiller, R.C., Fox, M.R., Gowland, P.A., 2015. Measurement of gastric meal and secretion volumes using magnetic resonance imaging. Physics in Medicine and Biology 60, 1367-1383.

Hogben, C.a., Schanker, L.S., Tocco, D.J., Brodie, B.B., 1957. Absorption of drugs from the stomach. II. The human. The Journal of pharmacology and experimental therapeutics 120, 540-545.

Holgate, A.M., Read, N.W., 1985. Effect of ileal infusion of intralipid on gastrointestinal transit, ileal flow rate, and carbohydrate absorption in humans after ingestion of a liquid meal. Gastroenterology 88, 1005-1011.

Holt, S., 1981. Observations on the relation between alcohol absorption and the rate of gastric emptying. Canadian Medical Association Journal 124, 267-277+297.

Horowitz, M., Maddern, G.J., Chatterton, B.E., Collins, P.J., Harding, P.E., Shearman, D.J., 1984. Changes in gastric emptying rates with age. Clinical science 67, 213-218.

Horowitz, M., Maddox, A.F., Wishart, J.M., Harding, P.E., Chatterton, B.E., Shearman, D.J., 1991. Relationships between oesophageal transit and solid and liquid gastric emptying in diabetes mellitus. European journal of nuclear medicine 18, 229-234.

Houghton, L.A., Mangnall, Y.F., Read, N.W., 1990. Effect of incorporating fat into a liquid test meal on the relation between intragastric distribution and gastric emptying in human volunteers. Gut 31, 12261229.

Houston, J.B., Levy, G., 1975. Effect of carbonated beverages and of an antiemetic containing carbohydrate and phosphoric acid on riboflavin bioavailability and salicylamide biotransformation in humans. Journal of pharmaceutical sciences 64, 1504-1507.

Hutson, W.R., Roehrkasse, R.L., Wald, A., 1989. Influence of gender and menopause on gastric emptying and motility. Gastroenterology 96, 11-17.

Indireshkumar, K., Brasseur, J.G., Faas, H., Hebbard, G.S., Kunz, P., Dent, J., Feinle, C., Li, M., Boesiger, P., Fried, M., Schwizer, W., 2000. Relative contributions of "pressure pump" and "peristaltic pump" to gastric emptying. American journal of physiology - Gastrointestinal and liver physiology 278, 604-616. Irving R, S., 1991. Polymers for Controlled Drug Delivery. 21, 296. 
Ito, Y., Okuda, S., Ohkawa, F., Kato, S., Mitsufuji, S., Yoshikawa, T., Takeuchi, K., 2008. Dual role of nitric oxide in gastric hypersecretion in the distended stomach: inhibition of acid secretion and stimulation of pepsinongen secretion. Life sciences $83,886-892$.

J. van der Schaar P., C.B.H.W.L., 1999. The Role of the Barostat in Human Research and Clinical Practice. Scandinavian journal of gastroenterology 34, 52-63.

Jain, A.K., Soderlind, E., Viriden, A., Schug, B., Abrahamsson, B., Knopke, C., Tajarobi, F., Blume, H., Anschutz, M., Welinder, A., Richardson, S., Nagel, S., Abrahmsen-Alami, S., Weitschies, W., 2014. The influence of hydroxypropyl methylcellulose (HPMC) molecular weight, concentration and effect of food on in vivo erosion behavior of HPMC matrix tablets. Journal of controlled release 187, 50-58.

Janssen, P., Verschueren, S., Ly, H.G., Vos, R., Van Oudenhove, L., Tack, J., 2011. Intragastric pressure during food intake: a physiological and minimally invasive method to assess gastric accommodation. Neurogastroenterology and motility 23, 316-322, e153-314.

Jaques, L.B., Hiebert, L.M., Wice, S.M., 1991. Evidence from endothelium of gastric absorption of heparin and of dextran sulfates 8000 . The Journal of laboratory and clinical medicine 117, 122-130.

Jaruratanasirikul, S., Kleepkaew, a., 1997. Influence of an acidic beverage (Coca-Cola) on the absorption of itraconazole. European journal of clinical pharmacology 52, 235-237.

Jobin, G., Cortot, a., Godbillon, J., Duval, M., Schoeller, J., Hirtz, J., Bernier, J., 1985. Investigation of drug absorption from the gastrointestinal tract of man. I. Metoprolol in the stomach, duodenum and jejunum. British journal of clinical pharmacology 19, 97S-105S.

Johnson, F.K., Ciric, S., Boudruai, S., Kisicki, J., Stauffer, J., 2012. Effects of alcohol on the pharmacokinetics of morhpine sulfate and naltrexone hydrochloride extended release. Journal of clinical pharmacology 52, 747-756.

Johnston, N., Dettmar, P.W., Bishwokarma, B., Lively, M.O., Koufman, J.A., 2007. Activity/stability of human pepsin: implications for reflux attributed laryngeal disease. The Laryngoscope 117, 1036-1039. Jones, B.E., 1988. Pharmaceutics: the science of dosage form design. Churchill Livingstone, p. 322.

Jones, K.L., Horowitz, M., Wishart, M.J., Maddox, A.F., Harding, P.E., Chatterton, B.E., 1995. Relationships between gastric emptying, intragastric meal distribution and blood glucose concentrations in diabetes mellitus. Journal of nuclear medicine 36, 2220-2228.

Jordan, N., Newton, J., Pearson, J., Allen, A., 1998. A novel method for the visualization of the in situ mucus layer in rat and man. Clinical science 95, 97-106.

Kalantzi, L., Goumas, K., Kalioras, V., Abrahamsson, B., Dressman, J.B., Reppas, C., 2006. Characterization of the human upper gastrointestinal contents under conditions simulating bioavailability/bioequivalence studies. Pharmaceutical research 23, 165-176.

Kamba, M., Seta, Y., Kusai, A., Ikeda, M., Nishimura, K., 2000. A unique dosage form to evaluate the mechanical destructive force in the gastrointestinal tract. International journal of pharmaceutics 208 , 61-70.

Karim, A., Burns, T., Wearley, L., Streicher, J., Palmer, M., 1985. Food-induced changes in theophylline absorption from controlled-release formulations. Part I. Substantial increased and decreased absorption with Uniphyl tablets and Theo-Dur Sprinkle. Clinical pharmacology and therapeutics 38, 7783.

Katelaris, P.H., Seow, F., Lin, B.P., Napoli, J., Ngu, M.C., Jones, D.B., 1993. Effect of age, Helicobacter pylori infection, and gastritis with atrophy on serum gastrin and gastric acid secretion in healthy men. Gut 34, 1032-1037.

Katori, N., Ma, W.S., Aoyagi, N., Kojima, S., 1996. Effect of destruction force on drug release from multiple unit controlled release dosage forms in humans. Pharmaceutical research 13, 1541-1546.

Kaye, J.L., 2011. Review of paediatric gastrointestinal physiology data relevant to oral drug delivery. International journal of clinical pharmacy 33, 20-24.

Kedzierewicz, F., Thouvenot, P., Lemut, J., Etienne, A., Hoffman, M., Maincent, P., 1999. Evaluation of peroral silicone dosage forms in humans by gamma-scintigraphy. Journal of Controlled Release 58, 195-205.

Kekki, M., Samloff, I.M., Ihamaki, T., Varis, K., Siurala, M., 1982. Age- and sex-related behaviour of gastric acid secretion at the population level. Scandinavian journal of gastroenterology 17, 737-743. 
Kenyon, C.J., Cole, E.T., Wilding, I.R., 1994. The effect of food on the in vivo behaviour of enteric coated starch capsules. International journal of pharmaceutics 112, 207-213.

Knupp, C.A., Milbrath, R.L., Barbhaiya, R.H., 1993a. Effect of metoclopramide and loperamide on the pharmacokinetics of didanosine in HIV seropositive asymptomatic male and female patients. European journal of clinical pharmacology 45, 409-413.

Knupp, C.a., Shyu, W.C., Morgenthien, E.a., Lee, J.S., Barbhaiya, R.H., 1993b. Biopharmaceutics of Didanosine in Humans and in a Model for Acid-Labile Drugs, the Pentagastrin-Pretreated Dog. Pharmaceutical research 10, 1157-1164.

Kossena, G.A., Charman, W.N., Wilson, C.G., O'Mahony, B., Lindsay, B., Hempenstall, J.M., Davison, C.L., Crowley, P.J., Porter, C.J.H., 2007. Low Dose Lipid Formulations: Effects on Gastric Emptying and Biliary Secretion. Pharmaceutical research 24, 2084-2096.

Kosugi, Y., Yamamoto, S., Sano, N., Furuta, A., Igari, T., Fujioka, Y., Amano, N., 2015. Evaluation of Acid Tolerance of Drugs Using Rats and Dogs Controlled for Gastric Acid Secretion. Journal of pharmaceutical sciences 104, 2887-2893.

Koziolek, M., Garbacz, G., Neumann, M., Weitschies, W., 2013. Simulating the postprandial stomach: physiological considerations for dissolution and release testing. Molecular pharmaceutics 10, 16101622.

Koziolek, M., Grimm, M., Garbacz, G., Kühn, J.-P., Weitschies, W., 2014a. Intragastric Volume Changes after Intake of a High-Caloric, High-Fat Standard Breakfast in Healthy Human Subjects Investigated by MRI. Mol. Pharmaceutics 11, 1632-1639.

Koziolek, M., Grimm, M., Garbacz, G., Kuhn, J.P., Weitschies, W., 2014b. Intragastric volume changes after intake of a high-caloric, high-fat standard breakfast in healthy human subjects investigated by MRI. Molecular pharmaceutics 11, 1632-1639.

Koziolek, M., Grimm, M., Becker, D., lordanov, V., Zou, H., Shimizu, J., Wanke, C., Garbacz, G., Weitschies, W., 2015a. Investigation of pH and Temperature Profiles in the GI Tract of Fasted Human Subjects Using the Intellicap ${ }^{\circledR}$ System. Journal of pharmaceutical sciences 104, 2855-2863.

Koziolek, M., Grimm, M., Becker, D., lordanov, V., Zou, H., Shimizu, J., Wanke, C., Garbacz, G., Weitschies, W., 2015b. Investigation of pH and Temperature Profiles in the GI Tract of Fasted Human Subjects Using the Intellicap((R)) System. Journal of pharmaceutical sciences 104, 2855-2863.

Koziolek, M., Schneider, F., Grimm, M., Modebeta, C., Seekamp, A., Roustom, T., Siegmund, W., Weitschies, W., 2015c. Intragastric $\mathrm{pH}$ and pressure profiles after intake of the high-caloric, high-fat meal as used for food effect studies. Journal of controlled release 220, 71-78.

Krishna, G., Moton, A., Ma, L., Medlock, M.M., McLeod, J., 2008. Pharmacokinetics and Absorption of Posaconazole Oral Suspension under Various Gastric Conditions in Healthy Volunteers. Antimicrobial Agents and Chemotherapy 53, 958-966.

Kristl, A., Vrečer, F., 2000. Preformulation Investigation of the Novel Proton Pump Inhibitor Lansoprazole. Drug development and industrial pharmacy 26, 781-783.

Kusano, M., Hosaka, H., Moki, H., Shimoyama, Y., Kawamura, O., Kuribayashi, S., Mori, M., Akuzawa, M., 2012. Cascade stomach is associated with upper gastrointestinal symptoms: a population-based study. Neurogastroenterology and motility 24, 451-455, e214.

Kwiatek, M.A., Steingoetter, A., Pal, A., Menne, D., Brasseur, J.G., Hebbard, G.S., Boesiger, P., Thumshirn, M., Fried, M., Schwizer, W., 2006. Quantification of distal antral contractile motility in healthy human stomach with magnetic resonance imaging. Journal of magnetic resonance imaging : 24, 1101-1109.

Kwiatek, M.A., Menne, D., Steingoetter, A., Goetze, O., Forras-Kaufman, Z., Kaufman, E., Fruehauf, H., Boesiger, P., Fried, M., Schwizer, W., Fox, M.R., 2009. Effect of meal volume and calorie load on postprandial gastric function and emptying: studies under physiological conditions by combined fiberoptic pressure measurement and MRI. American journal of physiology - Gastrointestinal and liver physiology 297, G894-901.

Lahner, E., Annibale, B., Delle Fave, G., 2009. Systematic review: impaired drug absorption related to the co-administration of antisecretory therapy. Alimentary pharmacology \& therapeutics 29, 12191229. 
Lakkireddy, D., Reddy, Y.M., Atkins, D., Rajasingh, J., Kanmanthareddy, A., Olyaee, M., Dusing, R., Pimentel, R., Bommana, S., Dawn, B., 2015. Effect of atrial fibrillation ablation on gastric motility: the atrial fibrillation gut study. Circulation. Arrhythmia and electrophysiology 8, 531-536.

Lange, D., Pavao, J.H., Wu, J., Klausner, M., 1997. Effect of a Cola Beverage on the Bioavailability of Itraconazole in the Presence of $\mathrm{H} 2$ blockers. The Journal of Clinical Pharmacology 37, 535-540.

Lannoo, M., Dillemans, B., 2014. Laparoscopy for primary and secondary bariatric procedures. Best practice \& research. Clinical gastroenterology 28, 159-173.

Lanza, F.L., 1984. Endoscopic Studies of Gastric and Duodenal Injury after the Use of Ibuprofen, Aspirin, and Other Nonsteroidal Anti-Inflammatory Agents. The American Journal of Medicine 77, 19-24.

Lee, K.W., Porter, C.J., Boyd, B.J., 2013. Gastric pre-processing is an important determinant of the ability of medium-chain lipid solution formulations to enhance oral bioavailability in rats. Journal of pharmaceutical sciences 102, 3957-3965.

Lennernas, H., Aarons, L., Augustijns, P., Beato, S., Bolger, M., Box, K., Brewster, M., Butler, J., Dressman, J., Holm, R., Julia Frank, K., Kendall, R., Langguth, P., Sydor, J., Lindahl, A., McAllister, M., Muenster, U., Mullertz, A., Ojala, K., Pepin, X., Reppas, C., Rostami-Hodjegan, A., Verwei, M., Weitschies, W., Wilson, C., Karlsson, C., Abrahamsson, B., 2014. Oral biopharmaceutics tools - time for a new initiative - an introduction to the IMI project OrBiTo. European journal of pharmaceutical sciences 57, 292-299.

Lennernäs, H., 2009. Ethanol-Drug Absorption Interaction: Potential for a Significant Effect on the Plasma Pharmacokinetics of Ethanol Vulnerable Formulations. Mol. Pharmaceutics 6, 1429-1440.

Levitt, M.D., Li, R., DeMaster, E.G., Elson, M., Furne, J., Levitt, D.G., 1997. Use of measurements of ethanol absorption from stomach and intestine to assess human ethanol metabolism. The American journal of physiology 273, G951-957.

Liang, J., Co, E., Zhang, M., Pineda, J., Chen, J.D., 1998. Development of gastric slow waves in preterm infants measured by electrogastrography. The American journal of physiology 274, G503-508.

Liau, S.-S., Camilleri, M., Kim, D.-Y., Stephens, D., Burton, D.D., O'Connor, M.K., 2001. Pharmacological modulation of human gastric volumes demonstrated noninvasively using SPECT imaging. Neurogastroenterology and motility 13, 533-542.

Lim, S., Kang, S.M., Shin, H., Lee, H.J., Won Yoon, J., Yu, S.H., Kim, S.-Y., Yoo, S.Y., Jung, H.S., Park, K.S., Ryu, J.O., Jang, H.C., 2011. Improved Glycemic Control Without Hypoglycemia in Elderly Diabetic Patients Using the Ubiquitous Healthcare Service, a New Medical Information System. Diabetes care 34, 308-313.

Lind, T., Cederberg, C., Ekenved, G., Haglund, U., Olbe, L., 1983. Effect of omeprazole -a gastric proton pump inhibitor -on pentagastrin stimulated acid secretion in man. Gut 24, 270-276.

Lindahl, A., Ungell, A.L., Knutson, L., Lennernas, H., 1997. Characterization of fluids from the stomach and proximal jejunum in men and women. Pharmaceutical research 14, 497-502.

Litou, C., Vertzoni, M., Goumas, C., Vasdekis, V., Xu, W., Kesisoglou, F., Reppas, C., 2016. Characteristics of the Human Upper Gastrointestinal Contents in the Fasted State Under Hypo- and A-chlorhydric Gastric Conditions Under Conditions of Typical Drug - Drug Interaction Studies. Pharmaceutical research 33, 1399-1412.

Lloyd, B.L., Greenblatt, D.J., Allen, M.D., Harmatz, J.S., Smith, T.W., 1978. Pharmacokinetics and bioavailability of digoxin capsules, solution and tablets after single and multiple doses. The American journal of cardiology 42, 129-136.

Lu, P.-J., Hsu, P.-I., Chen, C.-H., Hsiao, M., Chang, W.-C., Tseng, H.-H., Lin, K.-H., Chuah, S.-K., Chen, H.C., 2010. Gastric juice acidity in upper gastrointestinal diseases. World Journal of Gastroenterology 16, 5496-5501.

Luiking, Y.C., van der Reijden, A.C., van Berge Henegouwen, G.P., Akkermans, L.M., 1998. Migrating motor complex cycle duration is determined by gastric or duodenal origin of phase III. The American journal of physiology 275, G1246-1251.

Madsen, J.L., Graff, J., 2004. Effects of ageing on gastrointestinal motor function. Age and ageing 33, 154-159. 
Madsen, J.L., 2013. Scintigraphic assessment of gastrointestinal motility: a brief review of techniques and data interpretation. Clinical Physiology and Functional Imaging 34, 243-253.

Maes, B.D., Hiele, M.I., Geypens, B.J., Rutgeerts, P.J., Ghoos, Y.F., Vantrappen, G., 1994. Pharmacological modulation of gastric emptying rate of solids as measured by the carbon labelled octanoic acid breath test: influence of erythromycin and propantheline. Gut 35, 333-337.

Mahadevan, V., 2014. Anatomy of the stomach. Surgery (Oxford) 32, 571-574.

Malagelada, J.R., Longstreth, G.F., Summerskill, W.H., Go, V.L., 1976. Measurement of gastric functions during digestion of ordinary solid meals in man. Gastroenterology 70, 203-210.

Malhotra, S., Dixit, R.K., Garg, S.K., 2002. Effect of an acidic beverage (Coca-Cola) on the pharmacokinetics of carbamazepine in healthy volunteers. Methods and findings in experimental and clinical pharmacology 24, 31.

Marathe, P.H., Wen, Y., Norton, J., Greene, D.S., Barbhaiya, R.H., Wilding, I.R., 2001. Effect of altered gastric emptying and gastrointestinal motility on metformin absorption. British journal of clinical pharmacology 50, 325-332.

Marciani, L., Gowland, P.A., Spiller, R.C., Manoj, P., Moore, R.J., Young, P., Al-Sahab, S., Bush, D., Wright, J., Fillery-Travis, A.J., 2000. Gastric response to increased meal viscosity assessed by echoplanar magnetic resonance imaging in humans. The Journal of nutrition 130, 122-127.

Marciani, L., Gowland, P.A., Fillery-Travis, A., Manoj, P., Wright, J., Smith, A., Young, P., Moore, R., Spiller, R.C., 2001a. Assessment of antral grinding of a model solid meal with echo-planar imaging. American journal of physiology. Gastrointestinal and liver physiology 280, G844-849.

Marciani, L., Young, P., Wright, J., Moore, R., Coleman, N., Gowland, P.A., Spiller, R.C., 2001b. Antral motility measurements by magnetic resonance imaging. Neurogastroenterology and motility $13,511-$ 518.

Martinsen, T.C., Bergh, K., Waldum, H.L., 2005. Gastric juice: a barrier against infectious diseases. Basic \& clinical pharmacology \& toxicology 96, 94-102.

Marvola, M., Kannikoski, A., Aito, H., Nykänen, S., 1989. The effect of food on gastrointestinal transit and drug absorption of a multiparticular sustained-release verapamil formulation. International journal of pharmaceutics 53, 145-155.

Marzio, L., Giacobbe, A., Conoscitore, P., Facciorusso, D., Frusciante, V., Modoni, S., 1989. Evaluation of the use of ultrasonography in the study of liquid gastric emptying. The American journal of gastroenterology 84, 496-500.

Matzinger, D., 2000. The role of long chain fatty acids in regulating food intake and cholecystokinin release in humans. Gut 46, 689-694.

McArthur, K.E., Feldman, M., 1989. Gastric acid secretion, gastrin release, and gastric emptying in humans as affected by liquid meal temperature. The American journal of clinical nutrition 49, 51-54.

Meeroff, J.C., Go, V.L., Phillips, S.F., 1975. Control of gastric emptying by osmolality of duodenal contents in man. Gastroenterology 68, 1144-1151.

Menon, A., Ritschel, W.A., Sakr, A., 1994. Development and evaluation of a monolithic floating dosage form for furosemide. Journal of pharmaceutical sciences 83, 239-245.

Miller, R.A., 1941. Observations on the gastric acidity during the first month of life. Archives of disease in childhood 16, 22-30.

Minekus, M., 2015. The TNO Gastro-Intestinal Model (TIM), in: Verhoeckx, K., Cotter, P., LópezExpósito, I., Kleiveland, C., Lea, T., Mackie, A., Requena, T., Swiatecka, D., Wichers, H. (Eds.), The Impact of Food Bioactives on Health: in vitro and ex vivo models. Springer International Publishing, Cham, pp. 37-46.

Mitra, A., Kesisoglou, F., 2013. Impaired Drug Absorption Due to High Stomach pH: A Review of Strategies for Mitigation of Such Effect To Enable Pharmaceutical Product Development. Mol. Pharmaceutics 10, 3970-3979.

Miyaji, H., Azuma, T., Ito, S., Abe, Y., Ono, H., Suto, H., Ito, Y., Yamazaki, Y., Kohli, Y., Kuriyama, M., 1999. The effect of helicobacter pylori eradication therapy on gastric antral myoelectrical activity and gastric emptying in patients with non-ulcer dyspepsia. Alimentary pharmacology \& therapeutics 13 , 1473-1480. 
Mojaverian, P., Rocci, M.L., Conner, D.P., Abrams, W.B., Vlasses, P.H., 1987. Effect of food on the absorption of enteric-coated aspirin: Correlation with gastric residence time. Clinical pharmacology and therapeutics 41, 11-17.

Mojaverian, P., 1996. Evaluation of gastrointestinal pH and gastric residence time via the Heidelberg radiotelemetry capsule: Pharmaceutical application. Drug Dev. Res. 38, 73-85.

Moore, J.G., Tweedy, C., Christian, P.E., Datz, F.L., 1983. Effect of age on gastric emptying of liquidsolid meals in man. Digestive diseases and sciences 28, 340-344.

Moreau, H., Bernadac, A., Gargouri, Y., Benkouka, F., Laugier, R., Verger, R., 1989. Immunocytolocalization of human gastric lipase in chief cells of the fundic mucosa. Histochemistry 91, 419-423.

Morgan, K.G., Szurszewski, J.H., 1980. Mechanisms of phasic and tonic actions of pentagastrin on canine gastric smooth muscle. The Journal of physiology 301, 229-242.

Mudie, D.M., Murray, K., Hoad, C.L., Pritchard, S.E., Garnett, M.C., Amidon, G.L., Gowland, P.A., Spiller, R.C., Amidon, G.E., Marciani, L., 2014. Quantification of gastrointestinal liquid volumes and distribution following a $240 \mathrm{~mL}$ dose of water in the fasted state. Molecular pharmaceutics 11, 3039-3047.

Murakami, H., Matsumoto, H., Kubota, H., Higashida, M., Nakamura, M., Hirai, T., 2013. Evaluation of electrical activity after vagus nerve-preserving distal gastrectomy using multichannel electrogastrography. Journal of Smooth Muscle Research 49, 1-14.

Nakae, Y., Onouchi, H., Kagaya, M., Kondo, T., 1999. Effects of aging and gastric lipolysis on gastric emptying of lipid in liquid meal. Journal of gastroenterology 34, 445-449.

Nakagawa, Y., Itai, S., Yoshida, T., Nagai, T., 1992. Physicochemical Properties and Stability in the Acidic Solution of a New Macrolide Antibiotic, Clarithromycin, in Comparison with Erythromycin. Chemical \& Pharmaceutical Bulletin 40, 725-728.

Nefesoglu, F.Z., Ayanoglu-Dülger, G., Ulusoy, N.B., Imeryüz, N., 1998. Interaction of omeprazole with enteric-coated salicylate tablets. International journal of clinical pharmacology and therapeutics 36 , 549-553.

Neff, K.J., Olbers, T., le Roux, C.W., 2013. Bariatric surgery: the challenges with candidate selection, individualizing treatment and clinical outcomes. BMC medicine $11,8$.

Newton, J.L., Jordan, N., Oliver, L., Strugala, V., Pearson, J., James, O.F., Allen, A., 1998. Helicobacter pylori in vivo causes structural changes in the adherent gastric mucus layer but barrier thickness is not compromised. Gut 43, 470-475.

Newton, J.L., Jordan, N., Pearson, J., Williams, G.V., Allen, A., James, O.F., 2000. The adherent gastric antral and duodenal mucus gel layer thins with advancing age in subjects infected with Helicobacter pylori. Gerontology 46, 153-157.

Newton, J.M., 2010. Gastric emptying of multi-particulate dosage forms. International journal of pharmaceutics 395, 2-8.

Nimmo, J., Heading, R.C., Tothill, P., Prescott, L.F., 1973. Pharmacological Modification of Gastric Emptying: Effects of Propantheline and Metoclopromide on Paracetamol Absorption. BMJ 1, 587-589. Niv, Y., 2015. Helicobacter pylori and gastric mucin expression: A systematic review and meta-analysis. World journal of gastroenterology 21, 9430-9436.

Niwa, K., Jones, S.P., 1996. Preparation and Evaluation of a Time-Controlled Release Capsule Made of Ethylcellulose for Colon Delivery of Drugs. Journal of Drug Targeting 3, 477-477.

Notivol, R., Coffin, B., Azpiroz, F., Mearin, F., Serra, J., Malagelada, J.-R., 1995. Gastric tone determines the sensitivity of the stomach to distention. Gastroenterology 108, 330-336.

O'Connor, A., O'Morain, C., 2014. Digestive function of the stomach. Digestive diseases (Basel, Switzerland) 32, 186-191.

O'Donovan, D., Hausken, T., Lei, Y., Russo, A., Keogh, J., Horowitz, M., Jones, K.L., 2005. Effect of aging on transpyloric flow, gastric emptying, and intragastric distribution in healthy humans--impact on glycemia. Digestive diseases and sciences 50, 671-676.

O'Grady, G., Du, P., Cheng, L.K., Egbuji, J.U., Lammers, W.J., Windsor, J.A., Pullan, A.J., 2010. Origin and propagation of human gastric slow-wave activity defined by high-resolution mapping. American journal of physiology. Gastrointestinal and liver physiology 299, G585-592. 
Oberle, R.L., Chen, T.-S., Lloyd, C., Barnett, J.L., Owyang, C., Meyer, J., Amidon, G.L., 1990. The influence of the interdigestive migrating myoelectric complex on the gastric emptying of liquids. Gastroenterology 99, 1275-1282.

Oosterhuis, B., Jonkman, J.H., Andersson, T., Zuiderwijk, P.B., Jedema, J.N., 1991. Minor effect of multiple dose omeprazole on the pharmacokinetics of digoxin after a single oral dose. British journal of clinical pharmacology 32, 569-572.

Ostensen, H., Burhol, P.G., Heger, J., 1983. Gastric cascade: a diagnosis without clinical importance? Scandinavian journal of gastroenterology 18, 487-489.

Pabla, D., Akhlaghi, F., Zia, H., 2008. A comparative pH-dissolution profile study of selected commercial levothyroxine products using inductively coupled plasma mass spectrometry. European Journal of Pharmaceutics and Biopharmaceutics 72, 105-110.

Padwal, R., Brocks, D., Sharma, A.M., 2010. A systematic review of drug absorption following bariatric surgery and its theoretical implications. Obesity reviews 11, 41-50.

Padwal, R.S., Gabr, R.Q., Sharma, A.M., Langkaas, L.A., Birch, D.W., Karmali, S., Brocks, D.R., 2011. Effect of gastric bypass surgery on the absorption and bioavailability of metformin. Diabetes care 34, 1295-1300.

Pal, A., Indireshkumar, K., Schwizer, W., Abrahamsson, B., Fried, M., Brasseur, J.G., 2004. Gastric flow and mixing studied using computer simulation. Proceedings. Biological sciences / The Royal Society 271, 2587-2594.

Pal, A., Brasseur, J.G., Abrahamsson, B., 2007. A stomach road or "Magenstrasse" for gastric emptying. J Biomech 40, 1202-1210.

Pedersen, B.L., Mullertz, A., Brondsted, H., Kristensen, H.G., 2000. A comparison of the solubility of danazol in human and simulated gastrointestinal fluids. Pharmaceutical research 17, 891-894.

Pedersen, P.B., Vilmann, P., Bar-Shalom, D., Mullertz, A., Baldursdottir, S., 2013. Characterization of fasted human gastric fluid for relevant rheological parameters and gastric lipase activities. European journal of pharmaceutics and biopharmaceutics 85, 958-965.

Pedersen, P.B., Bar-Shalom, D., Baldursdottir, S., Vilmann, P., Müllertz, A., 2014. Feasibility of Capsule Endoscopy for Direct Imaging of Drug Delivery Systems in the Fasted Upper-Gastrointestinal Tract. Pharmaceutical research 31, 2044-2053.

Perri, F., Pastore, M.R., Annese, V., 2005. 13C-octanoic acid breath test for measuring gastric emptying of solids. European review for medical and pharmacological sciences. 9, 3-8.

Philip, A., Philip, B., 2010. Colon Targeted Drug Delivery Systems: A Review on Primary and Novel Approaches. Oman Medical Journal 25, 70-78.

Piche, M.E., Auclair, A., Harvey, J., Marceau, S., Poirier, P., 2015. How to choose and use bariatric surgery in 2015. The Canadian journal of cardiology 31, 153-166.

Pieramico, O., Dominguez-Muñoz, J.E., Nelson, D.K., Böck, W., Büchler, M., Malfertheiner, P., 1995. Interdigestive cycling in chronic pancreatitis: Altered coordination among pancreatic secretion, motility, and hormones. Gastroenterology 109, 224-230.

Pilbrant, Å., Cederberg, C., 1985. Development of an oral formulation of omeprazole. Scandinavian journal of gastroenterology 20, 113-120.

Piper, D.W., Fenton, B.H., 1965. pH stability and activity curves of pepsin with special reference to their clinical importance. Gut 6, 506-508.

Prescott, L.F., 1974. Gastric emptying and drug absorption. British journal of clinical pharmacology 1, 189-190.

Press, A.G., Hauptmann, I.A., Hauptmann, L., Fuchs, B., Fuchs, M., Ewe, K., Ramadori, G., 1998. Gastrointestinal pH profiles in patients with inflammatory bowel disease. Alimentary pharmacology \& therapeutics 12, 673-678.

Prewett, E.J., Smith, J.T., Nwokolo, C.U., Sawyerr, A.M., Pounder, R.E., 1991. Twenty-four hour intragastric acidity and plasma gastrin concentration profiles in female and male subjects. Clinical science $80,619-624$. 
Psachoulias, D., Vertzoni, M., Goumas, K., Kalioras, V., Beato, S., Butler, J., Reppas, C., 2011. Precipitation in and supersaturation of contents of the upper small intestine after administration of two weak bases to fasted adults. Pharmaceutical research 28, 3145-3158.

Quercia, I., Dutia, R., Kotler, D.P., Belsley, S., Laferrere, B., 2014. Gastrointestinal changes after bariatric surgery. Diabetes \& metabolism 40, 87-94.

Radwan, A., Amidon, G.L., Langguth, P., 2012. Mechanistic investigation of food effect on disintegration and dissolution of BCS class III compound solid formulations: the importance of viscosity. Biopharmaceutics \& drug disposition 33, 403-416.

Radwan, A., Ebert, S., Amar, A., Münnemann, K., Wagner, M., Amidon, G.L., Langguth, P., 2013. Mechanistic understanding of food effects: Water diffusivity in gastrointestinal tract is an important parameter for the prediction of disintegration of solid oral dosage forms. Molecular pharmaceutics 10, 2283-2290.

Radwan, A., Wagner, M., Amidon, G.L., Langguth, P., 2014. Bio-predictive tablet disintegration: Effect of water diffusivity, fluid flow, food composition and test conditions. European Journal of Pharmaceutical Sciences 57, 273-279.

Rajpurohit, H., Sharma, S., Sharma, P., Bhandari, a., 2010. Polymers for colon targeted drug delivery. Indian Journal of Pharmaceutical Sciences 72, 689.

Rakesh, T.P., 2011. Proton pump inhibitors: use, misuse and concerns about long-term therapy. Clinical journal of gastroenterology 4, 53-59.

Renou, C., Carriere, F., Ville, E., Grandval, P., Joubert-Collin, M., Laugier, R., 2001. Effects of lansoprazole on human gastric lipase secretion and intragastric lipolysis in healthy human volunteers. Digestion 63, 207-213.

Roberts, M., Cespi, M., Ford, J.L., Dyas, A.M., Downing, J., Martini, L.G., Crowley, P.J., 2007. Influence of ethanol on aspirin release from hypromellose matrices. International journal of pharmaceutics 332 , 31-37.

Robertson, D.R., Renwick, A.G., Wood, N.D., Cross, N., Macklin, B.S., Fleming, J.S., Waller, D.G., George, C.F., 1990. The influence of levodopa on gastric emptying in man. British journal of clinical pharmacology 29, 47-53.

Rodríguez-Fragoso, L., Martínez-Arismendi, J.L., Orozco-Bustos, D., Reyes-Esparza, J., Torres, E., Burchiel, S.W., 2011. Potential risks resulting from fruit/vegetable-drug interactions: effects on drugmetabolizing enzymes and drug transporters. Journal of food science 76, R112-124.

Rostami-Hodjegan, A., Shiran, M.R., Ayesh, R., Grattan, T.J., Burnett, I., Darby-Dowman, A., Tucker, G.T., 2002. A new rapidly absorbed paracetamol tablet containing sodium bicarbonate. I. A four-way crossover study to compare the concentration-time profile of paracetamol from the new paracetamol/sodium bicarbonate tablet and a conventional paracetamol tablet in fed and fasted volunteers. Drug development and industrial pharmacy 28, 523-531.

Rouge, N., Allémann, E., Gex-Fabry, M., Balant, L., Cole, E.T., Buri, P., Doelker, E., 1998. Comparative pharmacokinetic study of a floating multiple-unit capsule, a high-density multiple-unit capsule and an immediate-release tablet containing $25 \mathrm{mg}$ atenolol. Pharmaceutica Acta Helvetiae 73, 81-87.

Rubbens, J., Brouwers, J., Wolfs, K., Adams, E., Tack, J., Augustijns, P., 2016. Ethanol concentrations in the human gastrointestinal tract after intake of alcoholic beverages. European Journal of Pharmaceutical Sciences 86, 91-95.

Saad, R.J., Hasler, W.L., 2011. A Technical Review and Clinical Assessment of the Wireless Motility Capsule. Gastroenterology \& Hepatology 7, 795-804.

Sachdeva, P., Kantor, S., Knight, L.C., Maurer, A.H., Fisher, R.S., Parkman, H.P., 2013. Use of a high caloric liquid meal as an alternative to a solid meal for gastric emptying scintigraphy. Digestive diseases and sciences 58, 2001-2006.

Sanders, K.M., Koh, S.D., Ward, S.M., 2006. Interstitial cells of cajal as pacemakers in the gastrointestinal tract. Annual review of physiology 68, 307-343.

Sarosiek, I., Selover, K.H., Katz, L.A., Semler, J.R., Wilding, G.E., Lackner, J.M., Sitrin, M.D., Kuo, B., Chey, W.D., Hasler, W.L., Koch, K.L., Parkman, H.P., Sarosiek, J., McCallum, R.W., 2010. The assessment of 
regional gut transit times in healthy controls and patients with gastroparesis using wireless motility technology. Alimentary pharmacology \& therapeutics 31, 313-322.

Sasselli, V., Pachnis, V., Burns, A.J., 2012. The enteric nervous system. Developmental biology 366, 6473.

Sathyan, G., Xu, E., Thipphawong, J., Gupta, S.K., 2007. Pharmacokinetic profile of a 24-hour controlledrelease OROS formulation of hydromorphone in the presence and absence of food. BMC clinical pharmacology 7, 2.

Savoye, G., Savoye-Collet, C., Oors, J., Smout, A.J., 2003. Interdigestive transpyloric fluid transport assessed by intraluminal impedance recording. American journal of physiology - Gastrointestinal and liver physiology 284, G663-669.

Schanker, L.S., Shore, P.a., Brodie, B.B., Hogben, C.a., 1957. Absorption of drugs from the stomach. I. The rat. The Journal of pharmacology and experimental therapeutics 120, 528-539.

Schiller, C., FrÖHlich, C.P., Giessmann, T., Siegmund, W., MÖNnikes, H., Hosten, N., Weitschies, W., 2005. Intestinal fluid volumes and transit of dosage forms as assessed by magnetic resonance imaging. Alimentary pharmacology \& therapeutics 22, 971-979.

Schmidt, A.R., Buehler, P., Seglias, L., Stark, T., Brotschi, B., Renner, T., Sabandal, C., Klaghofer, R., Weiss, M., Schmitz, A., 2015. Gastric $\mathrm{pH}$ and residual volume after 1 and $2 \mathrm{~h}$ fasting time for clear fluids in children. British journal of anaesthesia 114, 477-482.

Schoofs, N., Devière, J., Van Gossum, a., 2006. PillCam colon capsule endoscopy compared with colonoscopy for colorectal tumor diagnosis: A prospective pilot study. Endoscopy 38, 971-977.

Schouten, R., Freijzer, P.L., Greve, J.W., 2007. Laparoscopic sleeve resection of a recurrent gastric cascade: a case report. Journal of laparoendoscopic \& advanced surgical techniques. Part A 17, 307310.

Schubert, M.L., Peura, D.A., 2008. Control of gastric acid secretion in health and disease. Gastroenterology 134, 1842-1860.

Schug, B., Brendel, E., Wonnemann, M., Wolf, D., Wargenau, M., Dingler, a., Blume, H., 2002a. Dosage form-related food interaction observed in a marketed once-daily nifedipine formulation after a highfat American breakfast. European journal of clinical pharmacology 58, 119-125.

Schug, B.S., Brendel, E., Chantraine, E., Wolf, D., Martin, W., Schall, R., Blume, H.H., 2002b. The effect of food on the pharmacokinetics of nifedipine in two slow release formulations: pronounced lag-time after a high fat breakfast. British journal of clinical pharmacology 53, 582-588.

Schug, B.S., Brendel, E., Wolf, D., Wonnemann, M., Wargenau, M., Blume, H.H., 2002c. Formulationdependent food effects demonstrated for nifedipine modified-release preparations marketed in the European Union. European Journal of Pharmaceutical Sciences 15, 279-285.

Schulze, K., 2006. Imaging and modelling of digestion in the stomach and the duodenum. Neurogastroenterology and motility 18, 172-183.

Seyberth, H.W., Kauffman, R.E., 2011. Basics and dynamics of neonatal and pediatric pharmacology. Handbook of experimental pharmacology 205, 3-49.

Shameem, M., Katori, N., Aoyagi, N., Kojima, S., 1995. Oral Solid Controlled Release Dosage Forms: Role of GI-Mechanical Destructive Forces and Colonic Release in Drug Absorption Under Fasted and Fed Conditions in Humans. Pharmaceutical research 12, 1049-1054.

Shih, G.L., Brensinger, C., Katzka, D.A., Metz, D.C., 2003. Influence of age and gender on gastric acid secretion as estimated by integrated acidity in patients referred for 24-hour ambulatory $\mathrm{pH}$ monitoring. The American journal of gastroenterology 98, 1713-1718.

Shirsand, S.B., Suresh, S., Swamy, P.V., Para, M.S., Nagendra Kumar, D., 2010. Formulation design of fast disintegrating tablets using disintegrant blends. Indian journal of pharmaceutical sciences 72,130 133.

Shukla, U.a., Pittman, K.a., Barbhaiya, R.H., 1992. Pharmacokinetic Interactions of Cefprozil With Food, Propantheline, Metoclopramide, and Probenecid in Healthy-Volunteers. Journal of clinical pharmacology 32, 725-731.

Siegel, J.A., Urbain, J.L., Adler, L.P., Charkes, N.D., Maurer, A.H., Krevsky, B., Knight, L.C., Fisher, R.S., Malmud, L.S., 1988. Biphasic nature of gastric emptying. Gut 29, 85-89. 
Simonian, H.P., Kantor, S., Linda C., K., Maurer, A.H., Megalooikonomou, V., Fisher, R.S., Parkman, H.P., 2003. Simultaneous assessment of gastric accommodation and emptying of solid and liquid meals. Gastroenterology 124, A53.

Simonian, H.P., Vo, L., Doma, S., Fisher, R.S., Parkman, H.P., 2005. Regional postprandial differences in $\mathrm{pH}$ within the stomach and gastroesophageal junction. Digestive diseases and sciences 50, 2276-2285. Singh, R., Singh, S., Lillard, J.W., 2008. Past, Present, and Future Technologies for Oral Delivery of Therapeutic Proteins. Journal of pharmaceutical sciences 97, 2497-2523.

Sirakov, V., Stefanov, R., Shipkov, C., 1994. The most common cause of cascade stomach. Folia medica 36, 37-39.

Skandalakis, L.J., Skandalakis, J.E., Skandalakis, P.N., 2009. Surgical Anatomy and Technique, Surgical Anatomy and Technique. Springer New York, pp. 285-332.

Skottheim, I.B., Stormark, K., Christensen, H., Jakobsen, G.S., Hjelmesaeth, J., Jenssen, T., Reubsaet, J.L., Sandbu, R., Asberg, A., 2009. Significantly altered systemic exposure to atorvastatin acid following gastric bypass surgery in morbidly obese patients. Clinical pharmacology and therapeutics 86, 311-318. Soons, P.a., van den Berg, G., Danhof, M., van Brummelen, P., Jansen, J.B.M.J., Lamers, C.B.H.W., Breimer, D.D., 1992. Influence of single- and multiple-dose omeprazole treatment on nifedipine pharmacokinetics and effects in healthy subjects. European journal of clinical pharmacology 42, 319324.

Soybel, D.I., 2005. Anatomy and physiology of the stomach. The Surgical clinics of North America 85, 875-894, v.

Stanghellini, V., Tosetti, C., Paternico, A., Barbara, G., Morselli-Labate, A.M., Monetti, N., Marengo, M., Corinaldesi, R., 1996. Risk indicators of delayed gastric emptying of solids in patients with functional dyspepsia. Gastroenterology 110, 1036-1042.

Stark, D.D., Bradley, W.G., Bradley, W.G., 2007. Magnetic Resonance Imaging. Radiologic Technology 78,551 .

Steffensen, G., Pedersen, S., 1986. Food induced changes in theophylline absorption from a once-aday theophylline product. British journal of clinical pharmacology 22, 571-577.

Stein, E., Clarke, J.O., Hutfless, S., Boult, C., Doggett, D., Fawole, O.A., Bass, E.B., 2013. Wireless Motility Capsule Versus Other Diagnostic Technologies for Evaluating Gastroparesis and Constipation: A Comparative Effectiveness Review. Comparative Effectiveness Reviews 110.

Steingoetter, A., Sauter, M., Curcic, J., Liu, D., Menne, D., Fried, M., Fox, M., Schwizer, W., 2015. Volume, distribution and acidity of gastric secretion on and off proton pump inhibitor treatment: a randomized double-blind controlled study in patients with gastro-esophageal reflux disease (GERD) and healthy subjects. BMC gastroenterology 15, 111.

Sternby, B., Hartmann, D., Borgstrom, B., Nilsson, A., 2002. Degree of in vivo inhibition of human gastric and pancreatic lipases by Orlistat (Tetrahydrolipstatin, $\mathrm{THL}$ ) in the stomach and small intestine. Clinical nutrition (Edinburgh, Scotland) 21, 395-402.

Streubel, A., Siepmann, J., Bodmeier, R., 2006. Gastroretentive drug delivery systems. Expert opinion on drug delivery 3, 217-233.

Sun, W.M., Houghton, L.A., Read, N.W., Grundy, D.G., Johnson, A.G., 1988. Effect of meal temperature on gastric emptying of liquids in man. Gut 29, 302-305.

Szafran, Z., Szafran, H., Popiela, T., Trompeter, G., 1978. Coupled Secretion of Gastric Lipase and Pepsin in Man following Pentagastrin Stimulation. Digestion 18, 310-318.

Tack, J., Piessevaux, H., Coulie, B., Caenepeel, P., Janssens, J., 1998. Role of impaired gastric accommodation to a meal in functional dyspepsia. Gastroenterology 115, 1346-1352.

Talattof, A., Price, J.C., Amidon, G.L., 2016. Gastrointestinal Motility Variation and Implications for Plasma Level Variation: Oral Drug Products. Molecular pharmaceutics.

Tappouni, H.L., Rublein, J.C., Donovan, B.J., Hollowell, S.B., Tien, H.-C., Min, S.S., Theodore, D., Rezk, N.L., Smith, P.C., Tallman, M.N., Raasch, R.H., Kashuba, A.D.M., 2008. Effect of omeprazole on the plasma concentrations of indinavir when administered alone and in combination with ritonavir. American Journal of Health-System Pharmacy 65, 422-428. 
Timmermans, J., Moes, a.J., 1994. Factors controlling the buoyancy and gastric retention capabilities of floating matrix capsules: New data for reconsidering the controversy. Journal of pharmaceutical sciences $83,18-24$.

Titus, R., Kastenmeier, A., Otterson, M.F., 2013. Consequences of gastrointestinal surgery on drug absorption. Nutrition in clinical practice 28, 429-436.

Tomomasa, T., Morikawa, A., Sandler, R.H., Mansy, H.A., Koneko, H., Masahiko, T., Hyman, P.E., Itoh, Z., 1999. Gastrointestinal sounds and migrating motor complex in fasted humans. The American journal of gastroenterology 94, 374-381.

Tonkic, A., Tonkic, M., Lehours, P., Megraud, F., 2012. Epidemiology and diagnosis of Helicobacter pylori infection. Helicobacter 17 Suppl 1, 1-8.

Tougas, G., Eaker, E.Y., Abell, T.L., Abrahamsson, H., Boivin, M., Chen, J., Hocking, M.P., Quigley, E.M., Koch, K.L., Tokayer, A.Z., Stanghellini, V., Chen, Y., Huizinga, J.D., Ryden, J., Bourgeois, I., McCallum, R.W., 2000. Assessment of gastric emptying using a low fat meal: establishment of international control values. The American journal of gastroenterology 95, 1456-1462.

Trowers, E., Tischler, M., 2014a. Form and Function: The Physiological Implications of the Anatomy of the Gastrointestinal System, Gastrointestinal Physiology. Springer International Publishing, pp. 9-35.

Trowers, E., Tischler, M., 2014b. Brain-Gut Axis and Regional Gastrointestinal Tract Motility, Gastrointestinal Physiology. Springer International Publishing, pp. 37-52.

Tseng, A., Szadkowski, L., Walmsley, S., Salit, I., Raboud, J., 2013. Association of age with polypharmacy and risk of drug interactions with antiretroviral medications in HIV-positive patients. The Annals of pharmacotherapy 47, 1429-1439.

Unger, M.M., Hattemer, K., Moller, J.C., Schmittinger, K., Mankel, K., Eggert, K., Strauch, K., Tebbe, J.J., Keil, B., Oertel, W.H., Heverhagen, J.T., Knake, S., 2010. Real-time visualization of altered gastric motility by magnetic resonance imaging in patients with Parkinson's disease. Movement disorders 25, 623-628.

Van Citters, G.W., Lin, H.C., 1999. The ileal brake: a fifteen-year progress report. Current gastroenterology reports 1, 404-409.

Van Den Abeele, J., Brouwers, J., Mattheus, R., Tack, J., Augustijns, P., 2015. Gastrointestinal Behavior of Weakly Acidic BCS Class II Drugs in Man - Case Study Diclofenac Potassium. Journal of pharmaceutical sciences, 687-696.

van den Berg, G., van Steveninck, F., Gubbens-Stibbe, J.M., Schoemaker, H.C., de Boer, a.G., Cohen, a.F., 1990. Influence of food on the bioavailability of metoprolol from an OROS system; a study in healthy volunteers. European journal of clinical pharmacology 39, 315-316.

van den Elzen, B.D.J., 2003. Fundic accommodation assessed by SPECT scanning: comparison with the gastric barostat. Gut 52, 1548-1554.

van der Schaar, P.J., Dijksman, J.F., Broekhuizen-de Gast, H., Shimizu, J., van Lelyveld, N., Zou, H., Iordanov, V., Wanke, C., Siersema, P.D., 2013. A novel ingestible electronic drug delivery and monitoring device. Gastrointestinal endoscopy 78, 520-528.

van Leeuwen, R.W.F., Peric, R., Hussaarts, K.G.a.M., Kienhuis, E., IJzerman, N.S., de Bruijn, P., van der Leest, C., Codrington, H., Kloover, J.S., van der Holt, B., Aerts, J.G., van Gelder, T., Mathijssen, R.H.J., 2016. Influence of the Acidic Beverage Cola on the Absorption of Erlotinib in Patients With Non-SmallCell Lung Cancer. Journal of Clinical Oncology, JCO.2015.2065.2560-.

Vantrappen, G., Janssens, J., Hellemans, J., Ghoos, Y., 1977. The interdigestive motor complex of normal subjects and patients with bacterial overgrowth of the small intestine. The Journal of clinical investigation 59, 1158-1166.

Vantrappen, G.R., Peeters, T.L., Janssens, J., 1979. The secretory component of the interdigestive migrating motor complex in man. Scandinavian journal of gastroenterology 14, 663-667.

Varum, F.J., Hatton, G.B., Basit, A.W., 2013. Food, physiology and drug delivery. International journal of pharmaceutics $457,446-460$.

Vaz-da-Silva, M., Loureiro, A.I., Nunes, T., Maia, J., Tavares, S., Falcão, A., Silveira, P., Almeida, L., Soares-da-Silva, P., 2005. Bioavailability and bioequivalence of two enteric-coated formulations of omeprazole in fasting and fed conditions. Clinical Drug Investigation 25, 391-399. 
Verhagen, M.A.M.T., Samsom, M., Smout, A.J.P.M., 1998. Gastric myoelectrical and antroduodenal motor activity in patients with achalasia. Neurogastroenterology and motility : the official journal of the European Gastrointestinal Motility Society 10, 211-218.

Vidon, N., Pfeiffer, A., Godbillon, J., Rongier, M., Gauron, S., Hirtz, J., Bernier, J.J., Dubois, J.P., 1989. Evaluation of the gastric absorption and emptying of drugs under various $\mathrm{pH}$ conditions using a simple intubation method: application to diclofenac. British journal of clinical pharmacology 28, 121-124.

Ville, E., Carriere, F., Renou, C., Laugier, R., 2002. Physiological study of pH stability and sensitivity to pepsin of human gastric lipase. Digestion 65, 73-81.

Vist, G.E., Maughan, R.J., 1995. The effect of osmolality and carbohydrate content on the rate of gastric emptying of liquids in man. The Journal of physiology 486 ( Pt 2), 523-531.

Vita, R., Saraceno, G., Trimarchi, F., Benvenga, S., 2014. Switching levothyroxine from the tablet to the oral solution formulation corrects the impaired absorption of levothyroxine induced by proton-pump inhibitors. The Journal of clinical endocrinology and metabolism 99, 4481-4486.

Walden, M., Nicholls, F.A., Smith, K.J., Tucker, G.T., 2007. The Effect of Ethanol on the Release of Opioids from Oral Prolonged-Release Preparations. Drug development and industrial pharmacy 33, 1101-1111.

Walravens, J., Brouwers, J., Spriet, I., Tack, J., Annaert, P., Augustijns, P., 2011. Effect of pH and comedication on gastrointestinal absorption of posaconazole: monitoring of intraluminal and plasma drug concentrations. Clinical pharmacokinetics 50, 725-734.

Weitschies, W., Cardini, D., Karaus, M., Trahms, L., Semmler, W., 1999. Magnetic marker monitoring of esophageal, gastric and duodenal transit of non-disintegrating capsules. Pharmazie 54, 426-430.

Weitschies, W., Karaus, M., Cordini, D., Trahms, L., Breitkreutz, J.r., Semmler, W., 2001. Magnetic marker monitoring of disintegrating capsules. European Journal of Pharmaceutical Sciences 13, 411416.

Weitschies, W., Kosch, O., Monikkes, H., Trahms, L., 2005a. Magnetic Marker Monitoring: An application of biomagnetic measurement instrumentation and principles for the determination of the gastrointestinal behavior of magnetically marked solid dosage forms. Advanced drug delivery reviews 57, 1210-1222.

Weitschies, W., Wedemeyer, R.-S., Kosch, O., Fach, K., Nagel, S., Söderlind, E., Trahms, L., Abrahamsson, B., Mönnikes, H., 2005b. Impact of the intragastric location of extended release tablets on food interactions. Journal of Controlled Release 108, 375-385.

Weitschies, W., Blume, H., Mönnikes, H., 2010. Magnetic Marker Monitoring: High resolution real-time tracking of oral solid dosage forms in the gastrointestinal tract. European Journal of Pharmaceutics and Biopharmaceutics 74, 93-101.

Westhus, N., 2004. Methods to test feeding tube placement in children. MCN. The American journal of maternal child nursing 29, 282-287; quiz 290-281.

WHO, 2015. Obesity and overweight. World Health Organization.

Wilde, L., Bock, M., Wolf, M., Glöckl, G., Garbacz, G., Weitschies, W., 2014. Development of pressuresensitive dosage forms with a core liquefying at body temperature. European Journal of Pharmaceutics and Biopharmaceutics 86, 507-513.

Yu, G., Zheng, Q.-S., Li, G.-F., 2014. Similarities and Differences in Gastrointestinal Physiology Between Neonates and Adults: a Physiologically Based Pharmacokinetic Modeling Perspective. The AAPS journal 16, 1162-1166.

Yuce, M., Dissanayake, T., Keong, H., 2009. Wireless telemetry for electronic pill technology. Sensors, 2009 IEEE.

Zhang, J., Ouyang, H., Zhu, H.B., Zhu, H., Lin, X., Co, E., Hayes, J., Chen, J.D., 2006. Development of gastric slow waves and effects of feeding in pre-term and full-term infants. Neurogastroenterology and motility : the official journal of the European Gastrointestinal Motility Society 18, 284-291.

Ziv, E., Bendayan, M., 2000. Intestinal absorption of peptides through the enterocytes. Microscopy research and technique $49,346-352$. 
Table 1. Composition of gastric fluids under fasted and fed state conditions

\begin{tabular}{|c|c|c|c|}
\hline & & Fasted state & Fed state \\
\hline $\begin{array}{c}\text { Volume } \\
-\end{array}$ & Mean residual volume (mL) & $\begin{array}{l}24 \pm 5 \text { (Dubois et al., 1977); } 35 \pm 22 \text { (Goetze } \\
\text { et al., 2009); } 31.4 \pm 19.7 \text { (Koziolek et al., } \\
\text { 2014b); } 35 \pm 7 \text { (Mudie et al., 2014); } 45 \pm 18 \\
\text { (Schiller et al., 2005) }\end{array}$ & $\begin{array}{l}\text { Dependent on meal volume } \\
\text { (Koziolek et al., 2014b; Kwiatek et al., } \\
\text { 2009) }\end{array}$ \\
\hline $\begin{array}{c}\text { Acidity } \\
-\end{array}$ & $\begin{array}{l}\mathrm{pH} \\
-\quad \text { Mean } \\
-\quad \text { Median } \\
\text { - } \quad \text { Range }\end{array}$ & 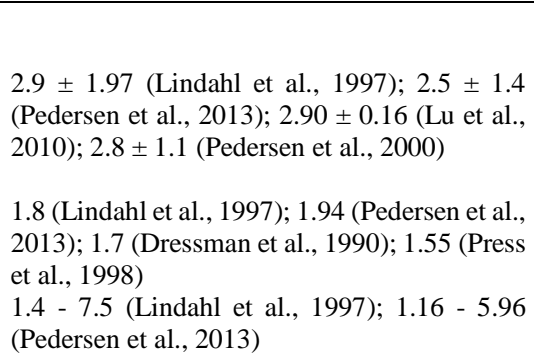 & 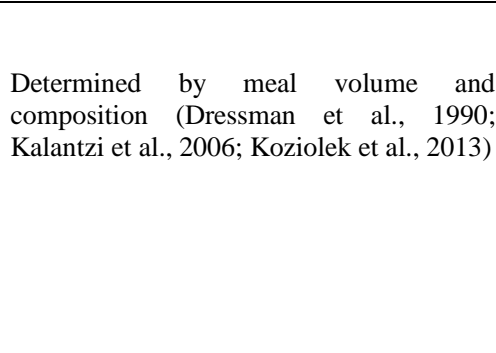 \\
\hline $\begin{array}{c}\text { Viscosity } \\
- \\
- \\
\end{array}$ & $\begin{array}{l}\text { Mean mucus layer thickness } \\
(\mu \mathrm{m})\end{array}$ & $\begin{array}{l}51.3 \pm 1.1 \text { (Al-Marhoon et al., 2005): } 110.5 \pm \\
37 \text { (Newton et al., 2000); } 106 \pm 30 \text { (Newton et } \\
\text { al., 1998); } 144 \pm 52 \text { (Jordan et al., 1998) } \\
1.7-9.3 \text { (Pedersen et al., 2013) }\end{array}$ & $\begin{array}{l}\text { Determined by meal viscosity: } \\
10 \text { - } 2000 \text { (Abrahamsson et al., 2005); } 466 \text { - } \\
2500 \text { (Radwan et al., 2012); 18.5 - 567 } \\
\text { (Radwan et al., 2014) }\end{array}$ \\
\hline $\begin{array}{c}\text { Enzymes } \\
-\end{array}$ & $\begin{array}{l}\text { Median pepsin concentration } \\
\text { (active; } \mathrm{mg} \cdot \mathrm{mL}^{-1} \text { ) }\end{array}$ & $0.11-0.22$ (Kalantzi et al., 2006) & $0.26-0.58$ (Kalantzi et al., 2006) \\
\hline- & $\begin{array}{l}\text { Gastric lipase activity } \\
\left(\mathrm{U} \cdot \mathrm{mL}^{-1}\right)\end{array}$ & $\begin{array}{l}7.4 \pm 4.0(\mathrm{pH} 2.8)(\text { Pedersen et al., } 2013) \\
99.0 \pm 45.3(\mathrm{pH} 5.4)(\text { Pedersen et al., } 2013)\end{array}$ & \\
\hline- & Amount secreted (mg) & & $\begin{array}{l}\text { liquid meal: } \\
21.6 \pm 14.5 \text { (total), } \\
14.5 \pm 10.8 \text { (active) (Carriere et al., 2001) } \\
15.4 \pm 8.0 \text { (active) (Renou et al., 2001) } \\
\text { mixed solid/liquid meal: } \\
15.2 \pm 5.1 \text { (total), } \\
13.2 \pm 4.6 \text { (active) (Carriere et al., 2001) } \\
14.7 \pm 8.7 \text { (active) (Carriere et al., 2005) }\end{array}$ \\
\hline Surface te & ension $(\mathrm{mN} / \mathrm{m})$ & $\begin{array}{l}34.8 \pm 5.2 \text { (Pedersen et al., 2013); } 35-45 \\
(\text { Efentakis and Dressman, 1998); } 41.9-45.7 \\
\left(250 \mathrm{~mL} \mathrm{H}_{2} \mathrm{O}\right)(\text { Kalantzi et al., 2006) }\end{array}$ & 30 - 31 (liquid meal) (Kalantzi et al., 2006) \\
\hline Bile salt c & oncentration $(\mathrm{mM})$ & $\begin{array}{l}0.2 \pm 0.5 \text { (Lindahl et al., 1997); } 0.33 \pm 0.31 \\
\text { (Pedersen et al., } 2013) ; 0.82 \pm 0.57 \text { (Pedersen } \\
\text { et al., 2000); 0.029 (Dewar et al., 1982); } \\
0.134 \pm 0.038 \text { (Foltz et al., 2015) }\end{array}$ & 0.022 (liquid meal) (Dewar et al., 1982) \\
\hline $\begin{array}{l}\text { Buffer ca } \\
\left(\mathrm{mmol} \cdot \mathrm{L}^{-1}\right.\end{array}$ & $\begin{array}{l}\text { pacity } \\
\left.. \Delta \mathrm{pH}^{-1}\right)\end{array}$ & $\begin{array}{l}14.3 \pm 9.5(\text { Pedersen et al., } 2013) \\
7-18\left(250 \mathrm{~mL} \mathrm{H}_{2} \mathrm{O}\right)(\text { Kalantzi et al., } 2006) \\
4.7-27.6\left(240 \mathrm{~mL} \mathrm{H}_{2} \mathrm{O}\right)(\text { Litou et al., } 2016)\end{array}$ & $\begin{array}{l}\text { liquid meal (Ensure Plus }{ }^{\circledR} \text { ): } \\
14 \text { - } 28 \text { (Kalantzi et al., 2006); } 22.5-30.0 \\
\text { (Diakidou et al., 2009) }\end{array}$ \\
\hline $\begin{array}{c}\text { Osmolalit } \\
-\end{array}$ & $\begin{array}{l}\text { Electrolytes }(\mathrm{mM}) \\
\text { Mean }\left(\mathrm{mOsm} \cdot \mathrm{kg}^{-1}\right)\end{array}$ & $\begin{array}{l}\mathrm{Na}^{+}: 68 \pm 29 ; \mathrm{K}^{+}: 13.4 \pm 3.0 ; \mathrm{Ca}^{2+}: 0.6 \pm 0.2 \\
\mathrm{Cl}^{-}: 102 \pm 28(\text { Lindahl et al., } 1997) \\
191 \pm 36 \text { (range: } 114-230)(\text { Lindahl et al., } \\
1997) \\
220 \pm 58(\text { Pedersen et al., } 2013) \\
98-140\left(250 \mathrm{~mL} \mathrm{H}_{2} \mathrm{O}\right)(\text { Kalantzi et al., } 2006) \\
44.9-144.0\left(240 \mathrm{~mL} \mathrm{H}_{2} \mathrm{O}\right)(\text { Litou et al., } 2016)\end{array}$ & $\begin{array}{l}\text { liquid meal }(610 \mathrm{mOsm} / \mathrm{kg}) \text { : } \\
559 \text { (Kalantzi et al., 2006) }\end{array}$ \\
\hline
\end{tabular}

